\title{
AN INTEGRATED ULTRASOUND TRANSDUCER DRIVER FOR HIFU APPLICATIONS
}

\author{
by \\ Wai Wong
A Thesis
Presented to Lakehead University
in Partial Fulfillment of the Requirement for the Degree of
Master of Science
in

Electrical and Computer Engineering

Thunder Bay, Ontario, Canada

September 19, 2013 


\begin{abstract}
Wai Wong, An Integrated Ultrasound Transducer Driver for HIFU Applications (Under the Supervision of Dr. Christoffersen, Dr. Curiel and Dr. Pichardo).

This thesis proposes an MRI-compatible integrated CMOS amplifier that is capable of directly driving an ultrasound transducer for HIFU applications. The output stage of the integrated amplifier operates in class DE mode with its output directly connected to a shunt capacitor and an ultrasound transducer without the need for an inductor. This design was simulated with Spectre $\AA$ simulator using the $0.8 \mu \mathrm{m}$ 5/20 V CMOS process data available from TeledyneDALSA Semiconductor. The proposed integrated amplifier has an efficiency of $80 \%$ with 1 W of output power at $1 \mathrm{MHz}$ and achieves an acceptable level of third harmonic. A layout of the integrated amplifier was prepared. The integrated amplifier occupies a die area of approximately $2.5 \mathrm{~mm}$ by $1.6 \mathrm{~mm}$ including input-output pads.
\end{abstract}




\section{Biographical Summary}

Wai Wong completed a BEng in Electrical Engineering in 2011 and is currently pursuing a master's degree in Electrical Engineering both from Lakehead University, Thunder Bay, Ontario. He has also served as a Graduate Assistant at his institution for two years. His research interests include analog circuit design and communications. He is a student member of the Institute of Electrical and Electronics Engineers (IEEE).

Part of this thesis has been published as the conference paper, "An Integrated ultrasound Transducer Driver for HIFU applications" which was presented at the 26th Annual Canadian Conference on Electrical and Computer Engineering, CCECE 2013, Regina, SK, hosted by the IEEE Canada in May 2013. 


\section{Acknowledgments}

This thesis would not have been possible without the support of Lakehead University, the Thunder Bay Regional Research Institute, the Natural Sciences and Engineering Research Council, and CMC Microsystems.

It gives me great pleasure in acknowledge Dr. Carlos Christoffersen, my supervisor, for providing support for this thesis, as well as his expertise in circuit design and the Cadence Design System, and his ideas and advice. I wish to thank Dr. Laura Curiel and Dr. Samuel Pichardo for sharing their in-depth knowledge of ultrasound transducers and High Intensity Focused Ultrasound.

I would also like to thank to Mr. Aaron Pearson for proofreading this thesis.

Finally, I thank my family for their encouragement, patience, and understanding.

Wai Wong

wlwong1@lakeheadu.ca 


\section{Contents}

$\begin{array}{ll}\text { List of Figures } & \text { v }\end{array}$

List of Tables $\quad$ ix

List of Symbols $\quad$ X

$\begin{array}{ll}\text { List of Abbreviations } & \mathbf{x}\end{array}$

1 Introduction $\quad 2$

1.1 Motivations and Objectives of this Study . . . . . . . . . . . . . . 2

2 Literature Review $\quad 4$

2.1 Introduction . . . . . . . . . . . . . . . . . . . 4

2.2 Review of Amplifier Topologies . . . . . . . . . . . . . . . . . . . . . . 4

2.2.1 Analog Amplifiers . . . . . . . . . . . . . . . 4

2.2.2 Switched Amplifiers . . . . . . . . . . . . . . . . . . 7

2.2.3 Other topologies for Switched Amplifier . . . . . . . . . . . . 13

2.3 Review of Published Designs . . . . . . . . . . . . . . . . 15

2.4 Summary . . . . . . . . . . . . . . . . . . . . . 24

3 Characterisation of an Ultrasound Transducer $\quad 27$

3.1 Transducer . . . . . . . . . . . . . . . . . . . 27

3.2 Equivalent Circuit . . . . . . . . . . . . . . . . . . . 28

3.3 Mathematical Model . . . . . . . . . . . . . . . . . . . . 29

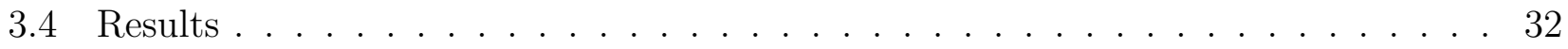


3.5 Ultrasound Field Characterization . . . . . . . . . . . . . . . . . . . . . . 34

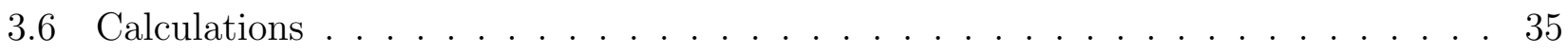

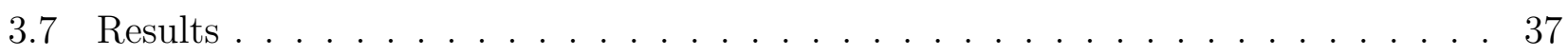

4 Amplifier Design $\quad 38$

4.1 Introduction . . . . . . . . . . . . . . . . . . 38

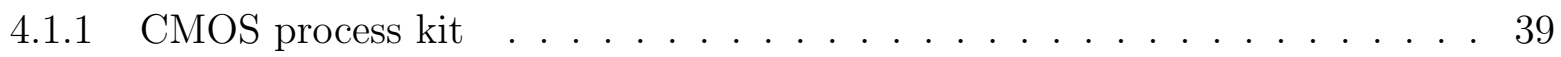

4.2 Class DE Amplifier . . . . . . . . . . . . . . . . . . . . . . . . . . . 39

4.2.1 Tuning of a practical class DE amplifier . . . . . . . . . . . . . 48

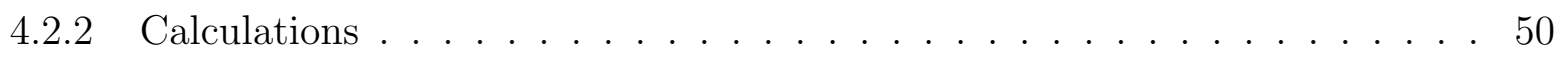

4.3 Gate Driver . . . . . . . . . . . . . . . . . . . 56

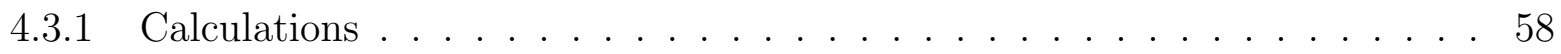

4.4 Current Mirror . . . . . . . . . . . . . . . . . . . . 60

4.5 Digital Block . . . . . . . . . . . . . . . . . . . 63

5 Simulation Results $\quad 66$

5.1 Pre-layout simulations . . . . . . . . . . . . . . . . 66

5.2 Post-layout simulations . . . . . . . . . . . . . . . . . . . . . . 70

6 Conclusions $\quad 74$

6.1 Future Work . . . . . . . . . . . . . . . . . . 75

$\begin{array}{ll}\text { A Transducer Housing } & 76\end{array}$

B Results of the Attenuation of Harmonics with $20 V_{p-p}$ Sinusoidal Signals 78

C An Estimation of M4's width using the Differential Equation Method $\quad 79$

$\begin{array}{lr}\text { D Schematic Diagram } & 82\end{array}$

$\begin{array}{lr}\text { E Layout View } & 92\end{array}$

$\begin{array}{lr}\text { References } & 106\end{array}$ 


\section{List of Figures}

2.1 Biasing points of classes $\mathrm{A}, \mathrm{B}$, and $\mathrm{AB}$ amplifiers [4] . . . . . . . . . 5

2.2 Block diagram of a single transistor $\mathrm{RF}$ amplifier $[5] \ldots \ldots \ldots$

2.3 Output waveforms of classes $\mathrm{A}, \mathrm{B}$ and $\mathrm{AB}$ amplifiers [5] . . . . . . . . 6

2.4 Class D half-bridge voltage switching amplifier $[5] \ldots \ldots \ldots \ldots$

2.5 Class D full-bridge voltage switching amplifier $[5] \ldots \ldots \ldots \ldots$

2.6 Voltage and current waveforms of class D full-bridge voltage switching amplifier [5]. 9

2.7 Topology of class E amplifier $[5] . \ldots \ldots \ldots \ldots \ldots$

2.8 Voltage and current waveforms of class E amplifier [5] . . . . . . . . . . . . 12

2.9 Topology of class DE amplifier with one shunt capacitor [5] . . . . . . . . . . 12

2.10 Topology of Step-up driving $[6] \ldots \ldots$. . . . . . . . . . . . . . . 13

2.11 Topology of flyback driving $[6] . \ldots \ldots \ldots \ldots \ldots \ldots$

2.12 Topology of Push-pull driving $[6] \ldots \ldots \ldots \ldots \ldots$

2.13 Schematic of DC to RF Inverter [7] . . . . . . . . . . . . . . . . . 16

2.14 Schematic of class D inverter for piezoelectric transducer [8] . . . . . . . . . 16

2.15 Magnitude impedance of piezoelectric ultrasound transducer[8] . . . . . . . . . 17

2.16 Schematic of switching amplifier and tuned filter $[2] \ldots \ldots \ldots \ldots$

2.17 Schematic of class D amplifier for Audio Beam System [9]. . . . . . . . . . . . . 19

2.18 Schematic of power inverter for harmonic cancellation [10] . . . . . . . . . . . 20

2.19 Waveform of harmonic cancellation $[10] . \ldots \ldots \ldots$

2.20 Schematic of class E amplifier with PFC [11]. . . . . . . . . . . . . 22

2.21 Schematic of switched-mode amplifier [12] . . . . . . . . . . . . . . . . 22

2.22 Schematic of output stage and matching network of LIPUS amplifier [13] . . . . . 23 
3.1 Exploded view of a transducer. . . . . . . . . . . . . . . . . 27

3.2 Equivalent circuit of a piezoelectric resonator near its resonance frequency [15] . . 28

3.3 Impedance of equivalent circuit in Figure $3.2[15] \ldots \ldots$. . . . . . . . . . . . . . . 29

3.4 Equivalent circuit of a transducer [14] . . . . . . . . . . . . . . . . . . 30

3.5 Comparison of the impedance of ultrasound transducer and equivalent circuit on a Smith Chart from $300 \mathrm{kHz}$ to $300 \mathrm{MHz}$. . . . . . . . . . . . . . . . . 31

3.6 Equivalent circuit of the piezoelectric resonator up to the 9th harmonic. . . . . . . 32

3.7 Comparison of the impedance of equivalent circuit and the measured results from VNA between $300 \mathrm{kHz}$ and $6 \mathrm{MHz} \ldots \ldots$. . . . . . . . . . . . . 33

3.8 Schematic diagram of the ultrasound field characterization using three-dimensional

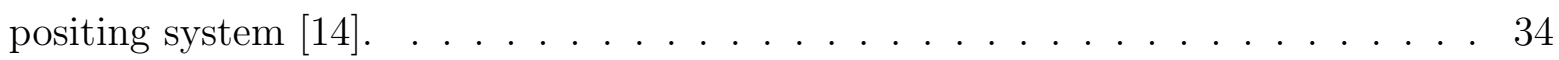

3.9 Schematic diagram of a voltage source driving a transducer through a coax [16]. . 35

4.1 Block diagram of the integrated amplifier for ultrasound transducer. . . . . . . . . 38

4.2 Symbol of high-voltage and low-voltage MOSFET used in this report. . . . . . . . 40

4.3 Topology of class DE amplifier with one shunt capacitor [5]. . . . . . . . . . 40

4.4 Voltage waveforms of class DE amplifier [5]. . . . . . . . . . . . . . . . 41

4.5 Current waveforms of class DE amplifier $[5] \ldots \ldots \ldots \ldots$. . . . . . . . . 42

4.6 Equivalent circuits of class DE amplifier at different stages [5] . . . . . . . . . . 44

4.7 Tuning procedure for class DE amplifier recommended by Albulet [19] . . . . . . . 49

4.8 Schematic of technological parameters extraction. . . . . . . . . . . . . . . 52

4.9 Square root of MOSFET drain current $\sqrt{I_{D}}$ as a function of gate-to-source voltage

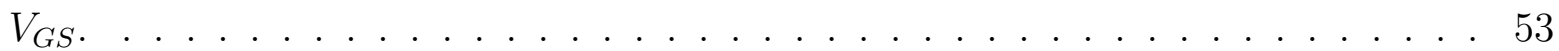

4.10 Simulation results of $\sqrt{I_{D}}$ as a function of $V_{G S} \ldots \ldots \ldots \ldots \ldots$

4.11 Schematic diagram of the gate driver for each power MOSFET [21] . . . . . . . 57

4.12 Schematic diagram of current mirror and current source [21] . . . . . . . . . . 60

4.13 Schematic diagram of the digital block. . . . . . . . . . . . . . . . . 64

4.14 Map for input functions and output of the digital block. . . . . . . . . . . . 65

5.1 Amplifier output voltage waveforms versus time at steady state. . . . . . . . . . 67

5.2 Drain current waveforms of M701 and M702 against time at steady state. . . . . . 67 
5.3 Discrete Fourier Transform of the amplifier's output voltage waveforms at steady

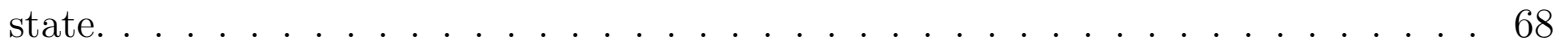

5.4 Percentage of third harmonic to fundamental of the amplifier output waveform as a function of switch-on duty ratio D with varying external capacitance at steady state. . . . . . . . . . . . . . . . . . . . . 69

5.5 Efficiency versus switch-on duty ratio D with a varying external capacitance at steady state. . . . . . . . . . . . . . . . . . . . 69

5.6 Output power against switch-on duty ratio with a varying external capacitance at steady state. . . . . . . . . . . . . . . . . . . 70

5.7 Layout of the integrated amplifier. . . . . . . . . . . . . . . . . . . . . . 72

5.8 Output voltage waveforms of pre- and post-layout simulations. . . . . . . . . . 73

5.9 Output current waveforms of pre- and post-layout simulations. . . . . . . . . . . 73

6.1 Projected percentage of third harmonic to fundamental in the acoustic pressure waveform as a function of switch-on duty ratio D with varying external capacitance. 75

A.1 Transducer Housing . . . . . . . . . . . . . . . . . . . . . . . . . . 77

D.1 Schematic diagram for simulating the integrated amplifier. . . . . . . . . . . . . 83

D.2 Top level schematic of the integrated amplifier. . . . . . . . . . . . . . . . . . . 84

D.3 Block diagram of the integrated amplifier. . . . . . . . . . . . . . . . 85

D.4 Block diagram of M701 gate driver. . . . . . . . . . . . . . . . . . . . 86

D.5 Schematic diagram of M701 gate driver. . . . . . . . . . . . . . . . . . . . 87

D.6 Block diagram of M702 gate driver. . . . . . . . . . . . . . . . . . . . . . 88

D.7 Schematic diagram of M702 gate driver. . . . . . . . . . . . . . . . . . . . . . 89

D.8 Schematic diagram of the digital block. . . . . . . . . . . . . . . . . 90

D.9 Schematic diagram of the ultrasound transducer. . . . . . . . . . . . . . . . . . . 91

E.1 Final integrated amplifier layout. . . . . . . . . . . . . . . . . . 93

E.2 Layout with M701 Gate driver. . . . . . . . . . . . . . . . . . . . . . . . . 94

E.3 Layout with M702 Gate driver. . . . . . . . . . . . . . . . . . . . . 95

E.4 Layout with M217 / M317. . . . . . . . . . . . . . . . . 96 


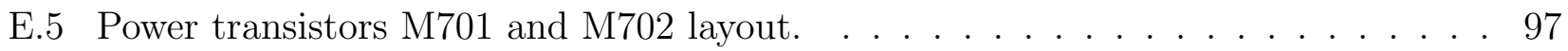

E.6 Layout with transistor M203. . . . . . . . . . . . . . . . . . . . . 98

E.7 Layout with transistor M204 _ . . . . . . . . . . . . . . . . . . . . 99

E.8 Layout with transistor M303. . . . . . . . . . . . . . . . . . . 100

E.9 Layout with transistor M304. . . . . . . . . . . . . . . . . . . . . 101

E.10 Layout with transistors M209 / M309 and M210 / 310 . . . . . . . . . . . . . 102

E.11 Layout with M211 - M214 / M311 - M314 . . . . . . . . . . . . . . . . 103

E.12 Layout with current mirror, M215 / M315 and M216 / M316. . . . . . . . . . . 104

E.13 Layout with combinational circuit block. . . . . . . . . . . . . . . . . . . 105 


\section{List of Tables}

2.1 Comparison of amplifier topologies. . . . . . . . . . . . . . . . . . 25

2.2 Comparison of specifications of published works. . . . . . . . . . . . 26

3.1 Attenuation of harmonics with $2 V_{p-p}$ sinusoidal signals. $\ldots \ldots \ldots \ldots$

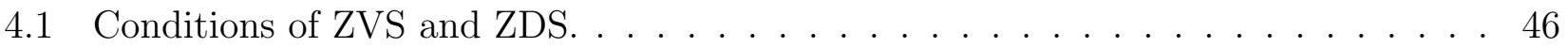

4.2 The expressions of class DE amplifier output voltage and current waveforms. . . . 47

4.3 A summary of the extracted technological parameters. . . . . . . . . . . . 55

4.4 Estimated size, gate capacitance, drain capacitance and channel resistance of transistors used in an earlier design. . . . . . . . . . . . . . . . . 5 55

4.5 Transistor dimensions in the final design. . . . . . . . . . . . . . . . . . 62

4.6 Truth Table. . . . . . . . . . . . . . . . . . . . . . . . . . . . 64

B.1 Attenuation of harmonics with $20 V_{p-p}$ sinusoidal signals. . . . . . . . . 78 


\section{List of Symbols}

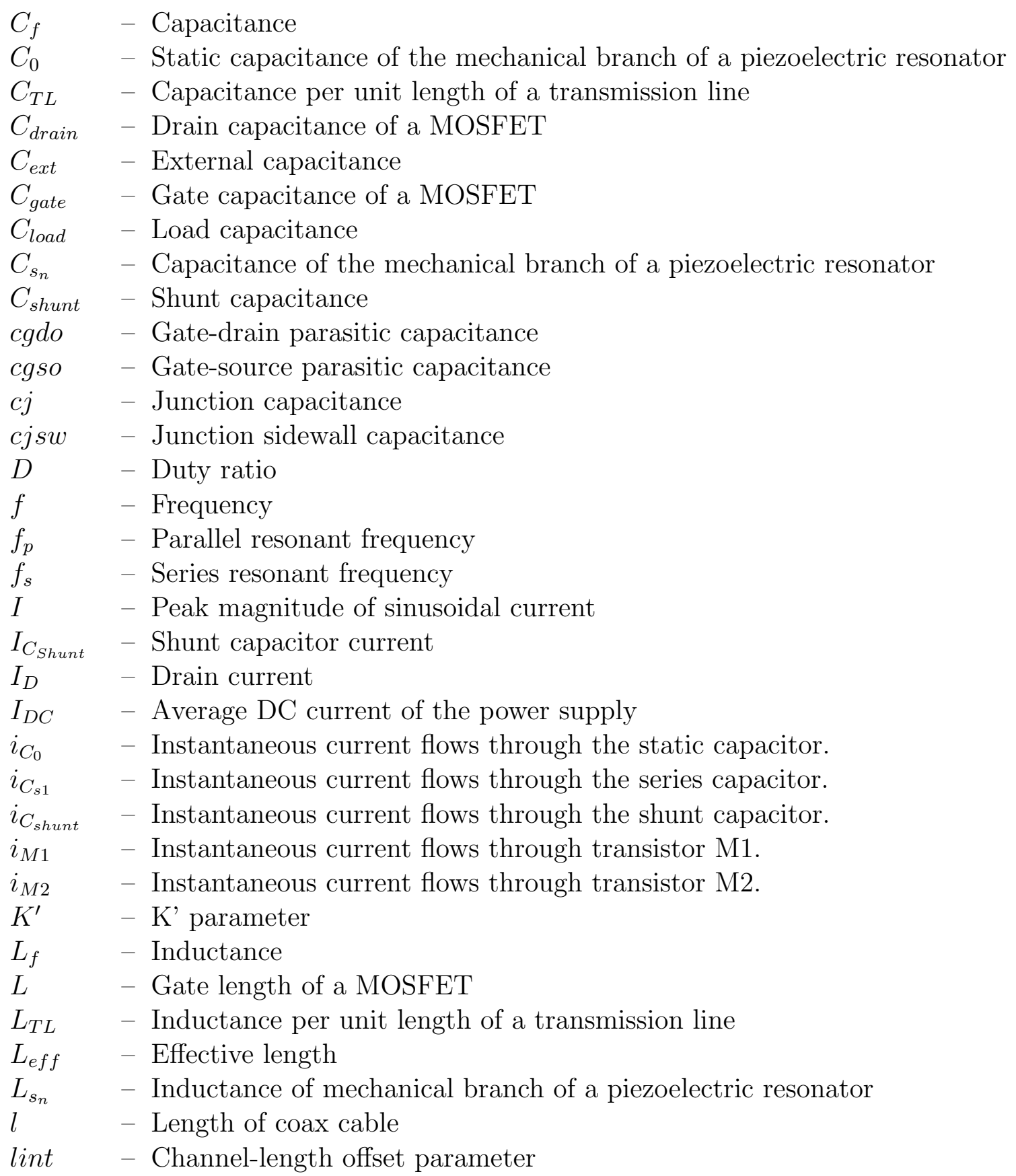




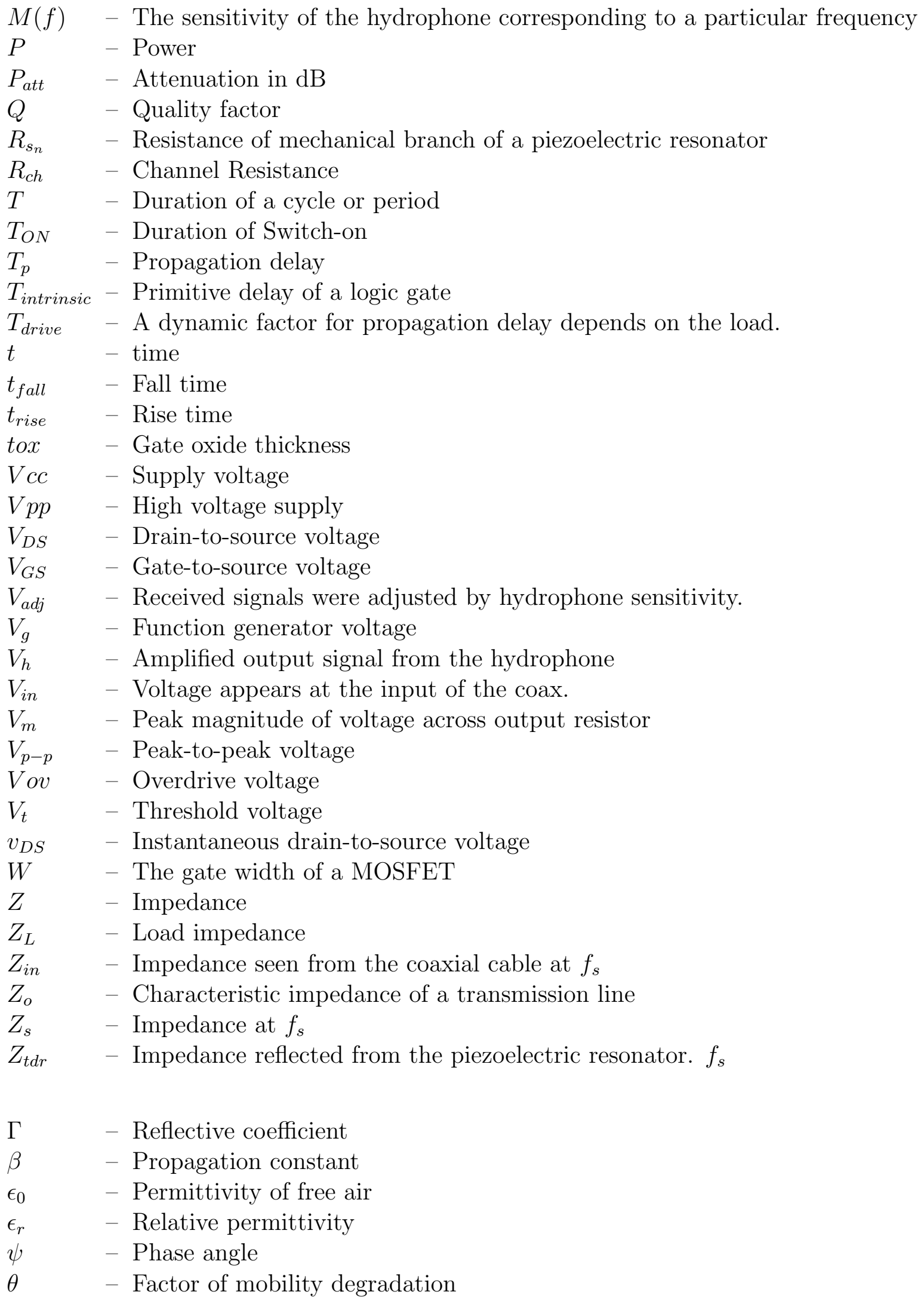


$2 \theta \quad-$ Angle of conduction

$\omega \quad-$ Angular frequency

\section{List of Abbreviations}

$\begin{array}{ll}\text { HIFU } & \text { - High Intensity Focused Ultrasound } \\ \text { HV } & \text { - High-Voltage } \\ \text { IC } & \text { - Integrated Circuit } \\ \text { LC } & \text { - Inductor and Capacitor } \\ \text { PZT } & \text { - Lead Zincorate Titaniate } \\ \text { LPF } & - \text { Low Pass Filter } \\ \text { LV } & \text { - Low-Voltage } \\ \text { MOSFET } & - \text { Metal Oxide Semiconductor Field Effect Transistor } \\ \text { NMOS } & - \text { N-type metal oxide semiconductor } \\ \text { PMOS } & - \text { P-type metal oxide semiconductor } \\ \text { PWM } & - \text { Pulse Width Modulation } \\ \text { RC } & \text { - Resistor and capacitor } \\ \text { RF } & \text { - Radio Frequency } \\ \text { RFC } & - \text { Radio Frequency Choke } \\ \text { TTL } & - \text { Transistor to Transistor Logic } \\ \text { VNA } & - \text { Vector Network Analyzer } \\ \text { ZVS } & \text { - Zero Voltage Switching } \\ \text { ZDS } & - \text { Zero Derivative Switching }\end{array}$




\section{Chapter 1}

\section{Introduction}

\subsection{Motivations and Objectives of this Study}

HIFU, or High Intensity Focused Ultrasound, is a non-invasive surgical technique that thermally ablates tissue in human organs, such as the liver, without the need of incision. Tumor ablation is achieved by focusing acoustic energy that translates into heat energy delivered to the focal zone of the ultrasound transducer [1]. As it is a very precise operation, any movements, such as respiration, of the patient's body may displace the focal zone and healthy tissue may be damaged. HIFU operation can be guided by Magnetic Resonance Imaging (MRI) so that tissue temperature can be monitored and any body movements can be compensated for in real time. Such development entails a multi-element HIFU transducer array that offers electronic focusing and sufficient pressure distribution, because it is necessary to produce a proper focal pattern without damaging the surrounding tissue [2]. Each element of an array is piloted by different phase information so as to create sufficient level of acoustic pressure at the focal zones. A sophisticated multi-element ultrasound transducer array may have over a thousand elements, which results in a very complex connections setup if the amplifier can not be kept close to the transducers. Therefore, downscaling the ultrasound therapy equipment becomes an interesting subject of study. The objective of this thesis is to design a CMOS power amplifier for a piezoelectric transducer to be installed near the piezoelectric resonator. Several of the challenges of designing the integrated amplifier are outlined below:

1. Each amplifier should occupy minimum possible area. 
2. The use of magnetic components, such as inductors, should be eliminated because they can interfere with the MRI's reception and distort the image [3].

3. To preclude overheating, the amplifier must be highly efficient.

4. It is capable of delivering $1 \mathbf{W}$ of power to the load with an acceptable level of harmonics.

\section{Thesis Overview}

Chapter 2 scrutinizes the applicability of analog amplifiers and switched amplifiers as well as typical published works on the subject of our project. Chapter 3 discusses the characterization of the ultrasound transducer and introduces a lump element model for the ultrasound transducer used in circuit simulation. Chapter 4 covers details of the circuit design, analysis of the class DE amplifier, methods of CMOS parameter extraction, and the design of transistors and gate drivers. Chapter 5 summarizes the simulation results, including output power, efficiency, ratio of third harmonic to fundamental, and a comparison of the pre-layout and post-layout simulations. Appendices D and E contain schematic diagrams and layout views of the proposed

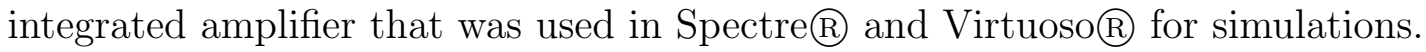




\section{Chapter 2}

\section{Literature Review}

\subsection{Introduction}

The original objective of this study was to review any antecedent published works on MRIcompatible HIFU integrated amplifiers. Unfortunately, published works on this topic were never found; furthermore, published works on integrated amplifier for ultrasound therapy are extremely scarce. As a result, this chapter reviews published works on piezoelectric amplifiers and scrutinizes their applicability to our problem. This chapter is organized as follows: Section 2.2 reviews

some basic topologies of analog amplifiers and switched amplifiers. Section 2.3 covers several published works that relate to the design of piezoelectric amplifiers. Section 2.4 summarizes the studies and concludes the chapter.

\subsection{Review of Amplifier Topologies}

\subsubsection{Analog Amplifiers}

Analog amplifiers, such as classes A, B, and AB, are distinguished by their biasing conditions and angles of conduction. Output transistors are controlled by gate-to-source voltage, $V_{G S}$ so that they are operating as dependent-current sources in saturation mode during amplification. Figure 2.1 shows the biasing conditions of class A, B, and AB amplifiers [4]. A general schematic is illustrated in Figure 2.2 [4]. 


\section{Tuned Network}

A tuned network offers the benefits of impedance matching, attenuation of harmonics, and improvement of efficiency [4]. It is inserted between the output of the amplifier and the load resistor as shown in Figure 2.2. A tuned filter can be designed with the following equation [4].

$$
f=\frac{1}{2 \pi \sqrt{L_{f} C_{f}}},
$$

where f denotes the cutoff frequency, $L_{f}$ stands for inductance and $C_{f}$ represents capacitance. In practice, filtering will cause additional losses because filters are implemented by non-ideal components.
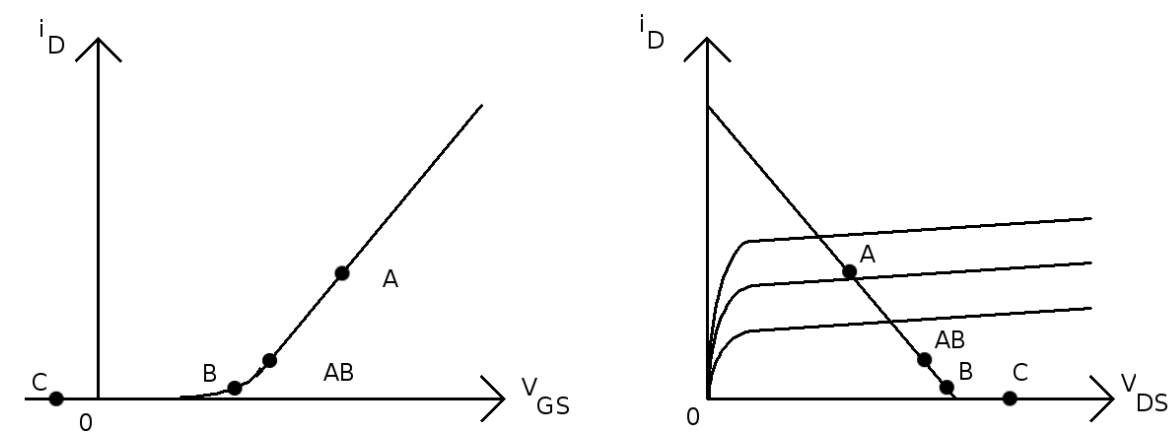

Figure 2.1: Biasing points of classes A, B, and AB amplifiers [4].

\section{Class A amplifier}

Class A operation denotes that an output transistor has an angle of conduction $2 \theta=2 \pi$ [5]. The gate-to-source voltage $V_{G S}$ of transistor M1 is much higher than threshold voltage $V_{t}$ and the drain-to-source voltage $V_{D S}$ is greater than $V_{G S}-V_{t}$ so as to maintain M1 in saturation mode during amplification. Therefore, class A operation is highly linear; it rarely needs any harmonic filters. The output waveform of a class A amplifier is shown in Figure 2.3. However, its maximum theoretical efficiency is $50 \%$ [4].

A class A amplifier demonstrates excellent linearity; however, poor efficiency becomes its major drawback. Without a proper cooling facility in place, high temperature can damage the surrounding circuitry instantly. Thus, a class A amplifier is not a sensible choice for an integrated 


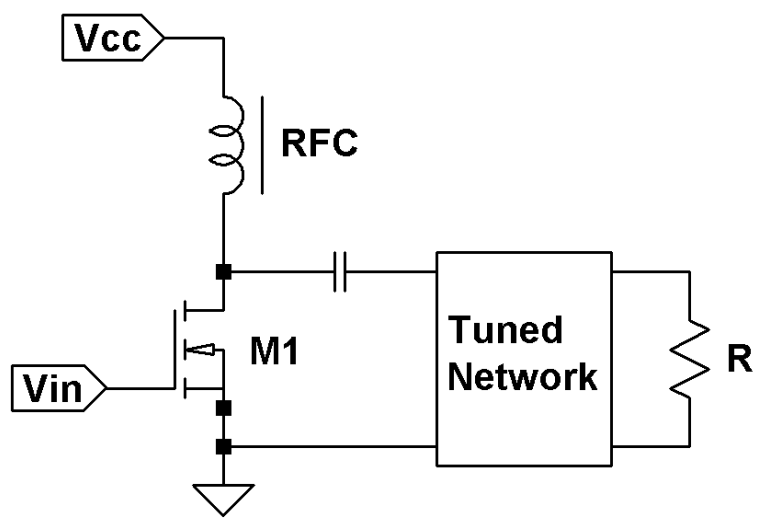

Figure 2.2: Block diagram of single transistor RF amplifier [5]. Note that: Choke can be eliminated when push-pull or rail-to-rail topologies are selected.

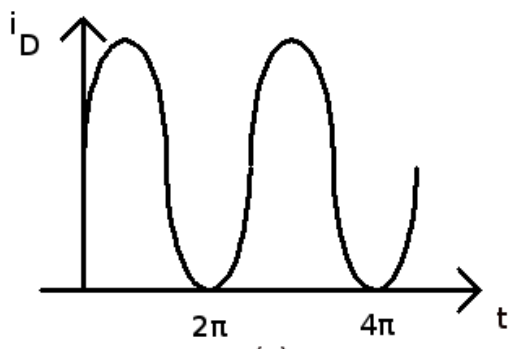

(a)

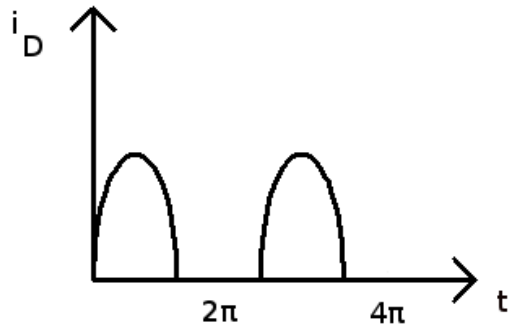

(b)

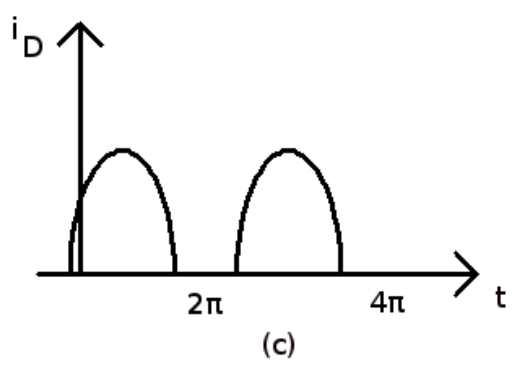

Figure 2.3: Output waveforms of (a) class A, (b) class B and (c) class AB amplifiers [5]. 
amplifier if sufficient cooling is unavailable.

\section{Class B amplifier}

Class B operation stands for an output transistor that has an angle of conduction $2 \theta=\pi$ [5]. The biasing voltage $V_{G S}$ of the power transistor is equal to $V_{t}$. In this case, transistor M1 enters active region when $V_{G S}>V_{t}$ and $V_{D S}>V_{G S}-V_{t}$. Therefore, only half of a period is being reproduced by the amplifier; this is shown in Figure 2.3. The quiescent bias current of a class B amplifier is equal to the threshold current.

The crucial challenges of class B amplifier include low efficiency and output distortion. The theoretical maximum efficiency of a class B amplifier is 78.5\% [4]. Moreover, since only the positive half of a sinusoidal waveform is being reproduced, complementary topology, such as pull-push, must be used. However, crossover distortion occurs when both transistors are not conducting.

\section{Class AB amplifier}

A class $\mathrm{AB}$ operation denotes an output transistor that has an angle of conduction $\pi \leq$ $2 \theta \leq 2 \pi[5]$. Voltage $V_{G S}$ is greater than or equal to $V_{t}$, and $V_{D S}>V_{G S}-V_{t}$. The theoretical maximum efficiency of a class AB amplifier is between $50 \%$ and $78.5 \%$, depending on the design. The output waveforms of class AB amplifier are shown in Figure 2.3.

Although a class AB amplifier offers a balance of efficiency and harmonics, similar to the class B amplifier, its theoretical maximum efficiency is too low for our needs.

\subsubsection{Switched Amplifiers}

For the remainder of this section, we will review several switched amplifiers that are potentially suitable for HIFU integrated amplifier design. Switched amplifiers are another group of power amplifiers that is commonly used in many applications due to their simple circuity and high efficiency. Unlike any analog amplifier, a switched amplifier operates its output transistors as switches. It is either transfers all voltage to the load when the switch is turned on or no voltage to the load when the switch is off. For that reason, the theoretical maximum efficiency is $100 \%$. In practice, the efficiency of a switched amplifier is limited by switching loss, non-zero 
channel resistance and harmonics [4].

\section{Class D amplifier}
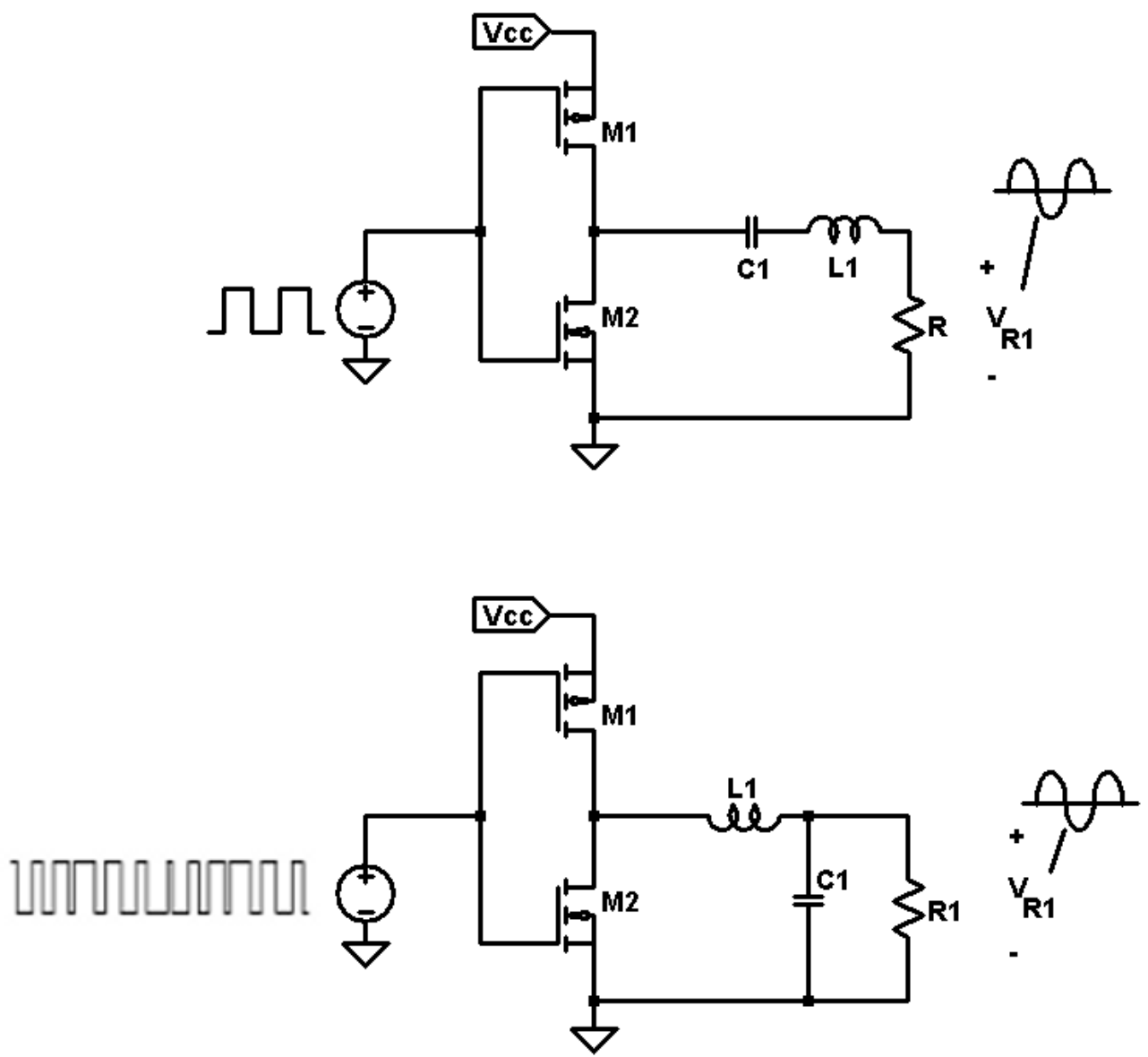

Figure 2.4: Class D half bridge voltage switching amplifier (a) Square wave, (b) PWM [5].

The schematic of the class D amplifier is shown in Figure 2.4 (a) [5]. Switches M1 and M2 will be turned on alternately at $50 \%$ duty cycle, to charge and discharge a tuned network. The drain terminals of M1 and M2 are connected to a tuned network that is responsible for attenuating the harmonics because the output waveforms from the amplifier are square waves. The theoretical maximum efficiency of a class D amplifier is 100\%; however, in practice, the efficiency is reduced by parasitic loss of the switches and filtering loss [4]. Furthermore, the design of the gate driver 


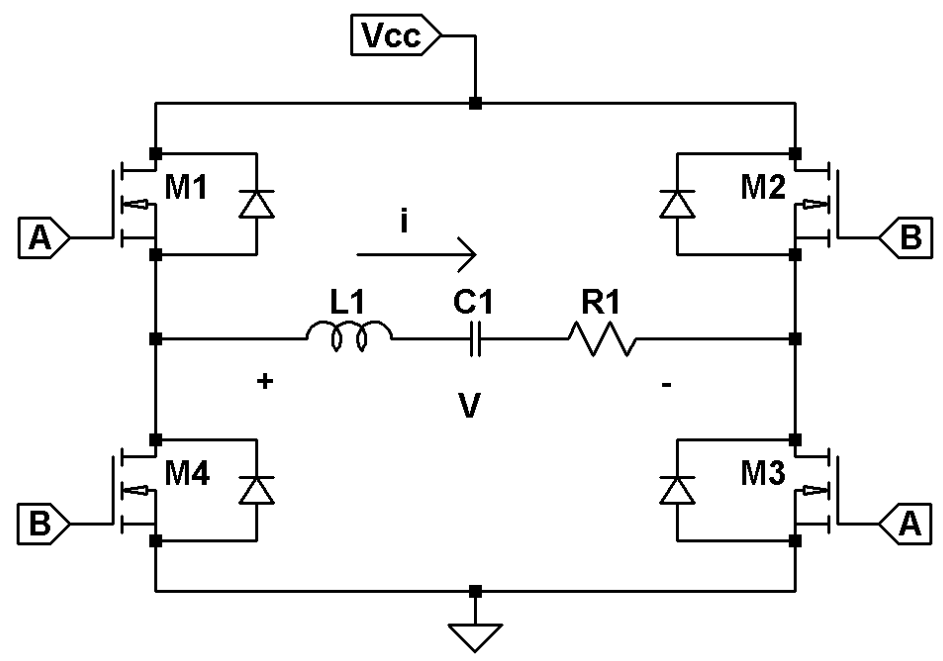

Figure 2.5: Topology of class D full bridge voltage switching amplifier [5].
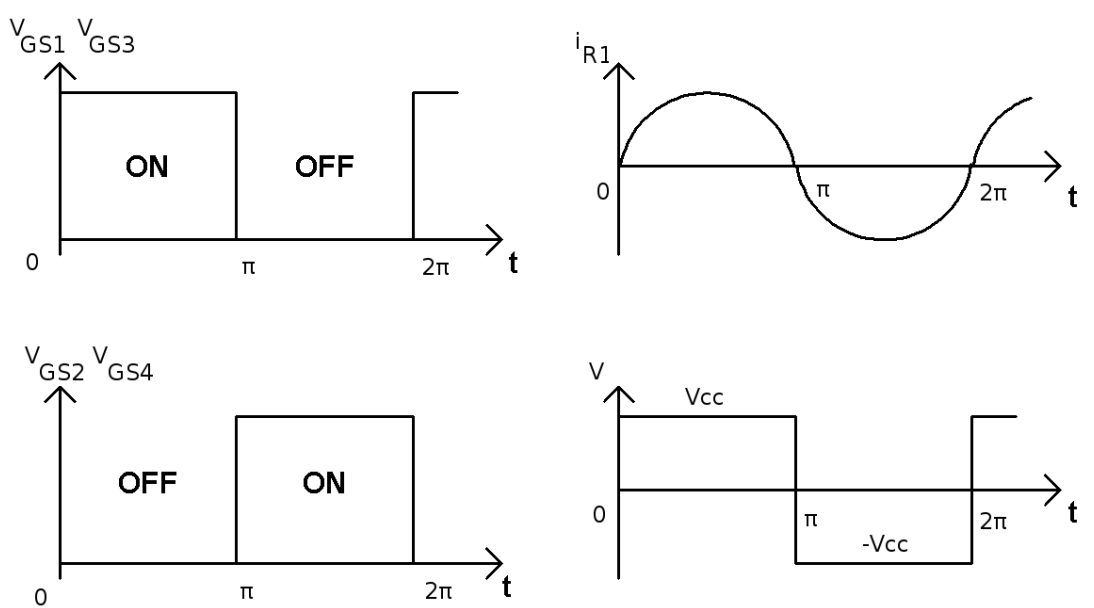

Figure 2.6: Waveforms of class D full bridge voltage switching amplifier (a) $V_{G S}$ of M1 and M3, (b) $V_{G S}$ of M2 and M4, (c) Current $i_{R}$, (d) Voltage V across series resonant circuit [5]. 
for the class D amplifier is usually complicated and costly because of the high voltage present at the terminal of M1 [6]. Moreover, interference generated by the power supply may be present at the driving circuit output [6].

Pulse width modulation (PWM) is another way of operating a class D amplifier. This idea is illustrated in Figure 2.4 (b). PWM is a modulation technique that converts the input signals to a series of square pulses with their duty ratios directly proportional to the electrical level of the input signals. The switching frequency of PWM should be at least 10 times higher than the frequency of input [4] [8]. The output low pass filter (LPF) serves multiple tasks including signal recovery, impedance matching, and noise reduction [8].

The class D full-bridge amplifier comprises four transistors and a tuned network. Its topology and waveforms are illustrated in Figure 2.5 and Figure 2.6 [5]. From 0 to $\pi$ radians, switches M1 and M3 conduct; M2 and M4 are opened. The voltage V across the series resonant circuit is $+V c c$. Between $\pi$ and $2 \pi$ radians, switches M2 and M4 conduct; M1 and M3 are opened. The voltage $\mathrm{V}$ becomes $-V c c$. Thus, the magnitude of output voltage is doubled because the direction of the current is manipulated by switches. The power delivered to the load R1 is quadrupled [5]. A class D full-bridge can also be driven by PWM.

In general, a class D amplifier should be operated beyond the resonant frequency of the tuned network. This is because current spikes created by the leading current of a capacitive load with respect to the voltage can cause a breakdown of a parasitic bipolar transistor inside a MOSFET during an on-off transition [5]. To alleviate the situation, several measures can be taken, such as using snubber circuits, connecting an inductor in series with MOSFETs, choosing high-voltage, or high on-resistance transistors [5].

The class D half-bridge amplifier is a very popular choice of output stage for ultrasound therapy applications [2] [12] [13]. For class D half and full bridge amplifiers, the output voltages are square waves which are unsuitable for transducers. A tuned filter must be used to attenuate harmonics because the output waveforms are square waves. The maximum efficiency drops to $81 \%$ if there is no filtering in place [4]; this can be verified by dividing the power of the fundamental to the power of the square wave. Although full bridge configuration offers higher output power, it also doubles the output parasitic capacitance and the occupied area.

Using PWM with a class D amplifier for driving piezoelectric transducers requires a low pass 
filter to remove high frequency components. Therefore, this technique has the best chance of recovering the original signals. However, the harmonics of the PWM's switching frequency can interfere with the MRI's reception. In practice, parasitic loss of MOSFETs increases with the increase of switching frequency [13].

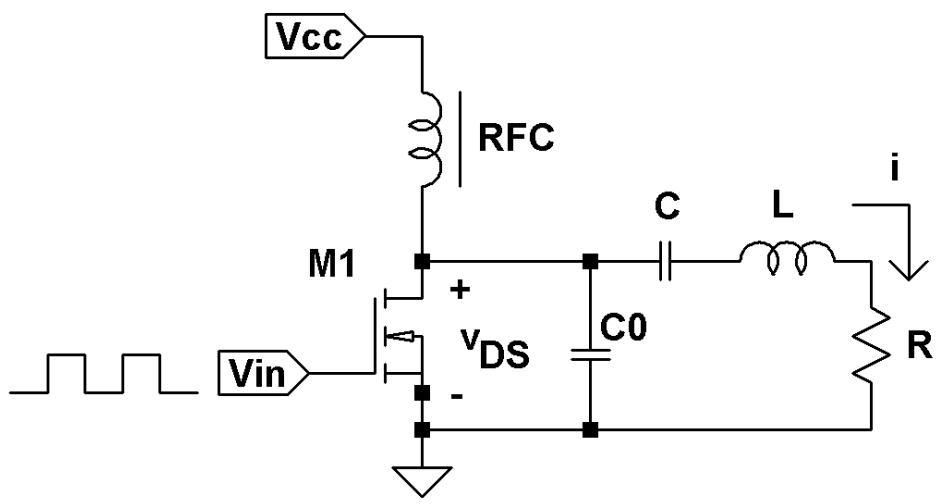

Figure 2.7: Topology of class E amplifier [5].

\section{Class E amplifier}

A class E amplifier is a single switch amplifier with a theoretical efficiency of $100 \%$. Figure 2.7 and Figure 2.8 illustrate its topology and waveforms. Unlike with the other amplifiers mentioned above, the tuned network is part of the class E amplifier. Tuned network allows $v_{D S}$ to slowly decline to zero exactly at the moment when M1 conducts. Since voltage $v_{D S}$ across the switch is zero, the switching loss is also zero. It is called zero-voltage-switching ZVS [5]. In addition, the derivative of $v_{D S}$ is also zero at the point where ZVS takes place; this is called zero-derivativeswitching [5], ZDS. Reference [11] is an example of using a class E amplifier to drive a piezoelectric transducer.

The major drawback of a class E amplifier is the need for an RF choke that is used to limit the current ripples generated by switching. For $10 \%$ current ripples, the minimum inductance of the choke is approximately 8.7 times the series resistance Rs, divided by the switching frequency [5]. So, it is not a suitable solution for an integrated amplifier, because the available room for the die is very limited. 

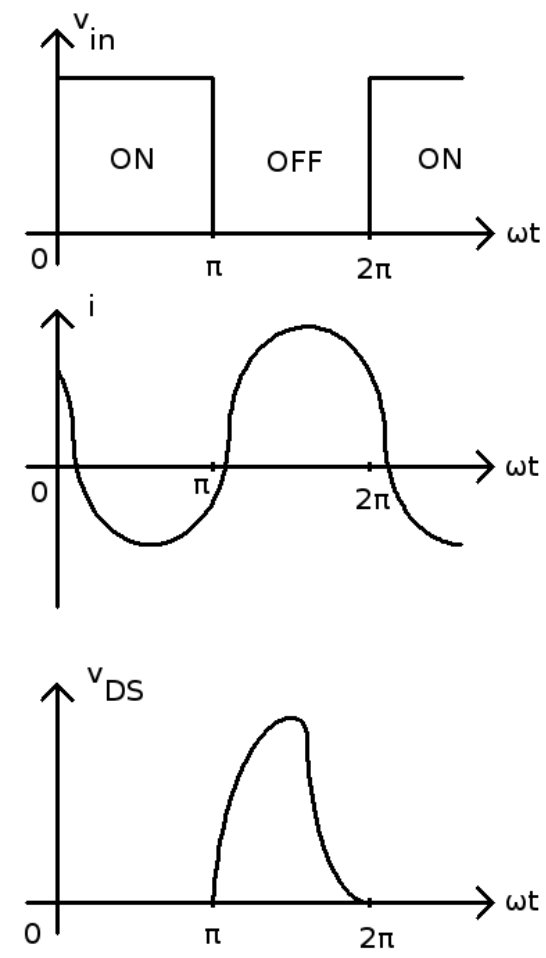

Figure 2.8: Waveforms of Class E amplifier. (a) On-off signals, (b) Current waveforms through resistor, (c) Drain-to-source voltage waveforms [5].

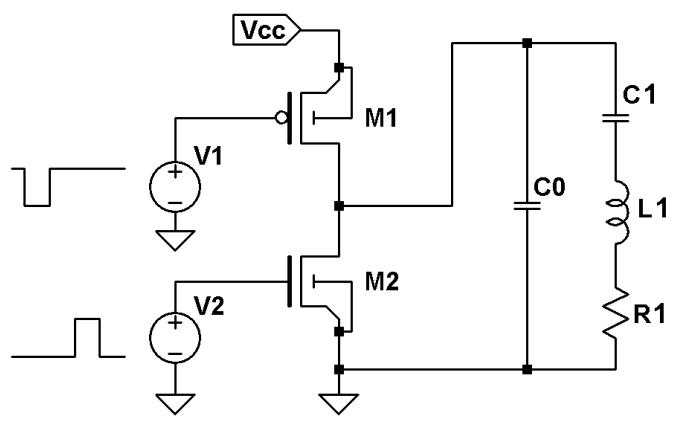

Figure 2.9: Topology of class DE amplifier with one shunt capacitor [5]. 


\section{Class DE amplifier}

A class DE amplifier integrates class D amplifier topology and class E switching condition. It has a theoretical efficiency of $100 \%$. Figure 2.9 illustrates the basic topology of a class DE amplifier. Switches M1 and M2 turn on and off alternately with a duty ratio of 0.25 ; therefore, two time gaps of $0.25 \mathrm{~T}$ are created between pulses to allow ZVS and ZDS to take place. A detailed analysis of the class DE amplifier is provided in Chapter 4.

Much as other switching amplifiers, the output waveform of a class DE amplifier contains a moderate level of harmonics; additional filtering will be required for HIFU applications. Since it is a switched amplifier, parasitic loss appears when ZVS and ZDS do not perform precisely.

\subsubsection{Other topologies for Switched Amplifier}

\section{Step-up topology}

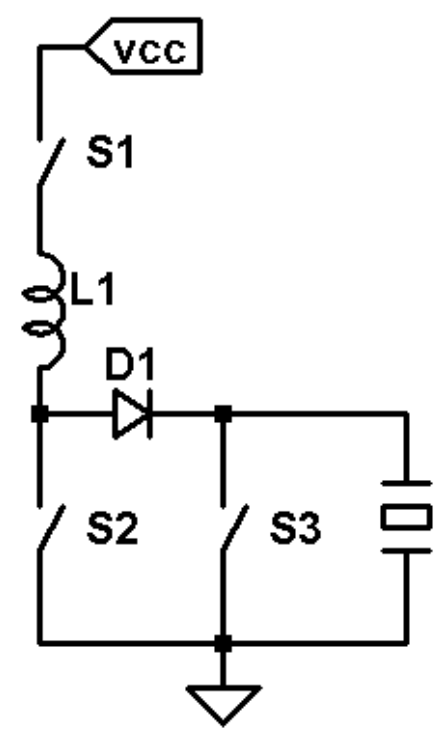

Figure 2.10: Topology of Step-up driving [6].

The schematic of the step-up driving topology is shown in Figure 2.10 [6]. Switches S1 and S2 charge up the inductor. Then, the energy stored in inductor transfers to the transducer when $\mathrm{S} 2$ is switched off. At last, S1 switches off and S3 discharges the transducer [6].

Since the step-up topology employs three switches, its timing and control circuits can be 
very complicated. Moreover, diode D1 introduces additional losses.

\section{Flyback topology}

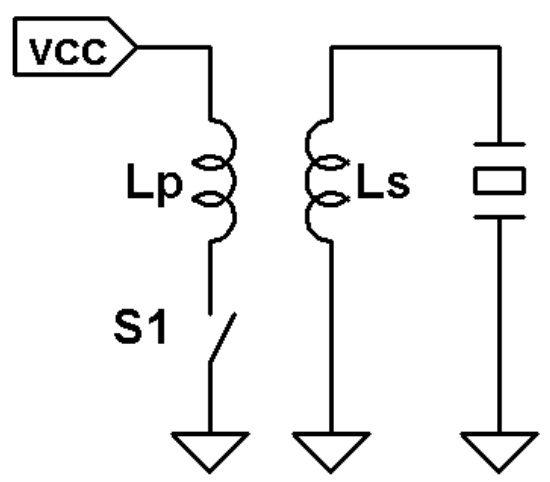

Figure 2.11: Topology of flyback driving [6].

The schematic of the flyback topology is shown in Figure 2.11. The primary winding of the flyback transformer connects to the supply voltage and switch. By turning switch S1 on and off, a high voltage is created at the terminal of S1. The secondary winding of transformer ties to ground and transducer.

The major advantage of a flyback topology over other circuit topologies is that it needs only one switch to generate signal pulses. However, the secondary winding and the static capacitor of the transducer may create ringing [6].

\section{Push-pull topology}

The schematic of the push-pull topology is shown in Figure 2.12. The low voltage supply ties to the central tap of transformer. Switches S1 and S2 connect to the primary winding of transformer. Each switch turns on and off alternatively with a duty ratio of 0.5. The secondary winding attaches to the transducer. The transformer is used for matching impedance of the power supply and transducer to improve efficiency [6].

This topology is unsuitable for our design because it employs a transformer. Since the integrated amplifier occupies an area of few millimeters, a transformer is too large for our appli- 


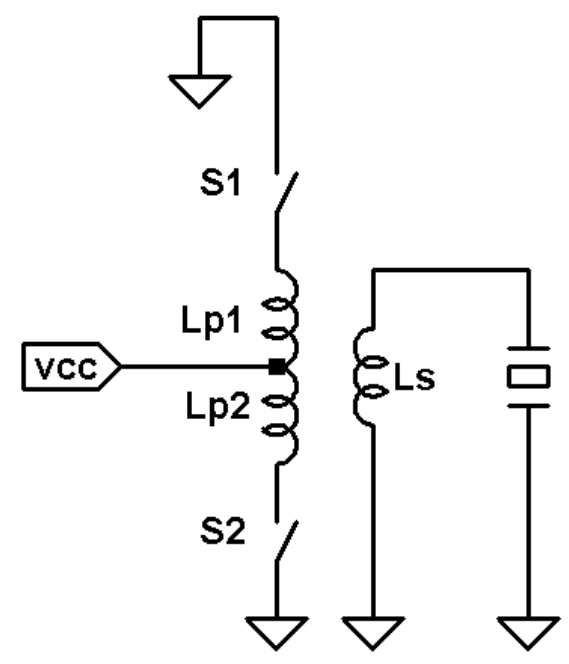

Figure 2.12: Topology of Push-pull driving [6].

cations.

Table 2.1 on Page 25 summarizes the key features of the different types of amplifier introduced in this section for comparison.

\subsection{Review of Published Designs}

The second half of this chapter dissertates the published amplifier topology for a piezoelectric ultrasound transducer. Precedence of publications is organized chronologically.

Mizutani et al. [7] proposed a $60 \mathrm{~W}, 1 \mathrm{MHz}$ DC-RF inverter that automatically tuned its operating frequency to the resonant frequency of the transducer. The schematic is shown in Figure 2.13. The purpose of implementing auto-tuning is to minimize the phase angle of output voltage and current to the transducer so as to maintain a resistive output impedance. Current sensing is implemented by feeding the current signal to a band pass filter and phase lock loop.

This is the flyback topology. It employs an output transformer and a current transformer. As a result, it is not suitable for our design.

Agbossou et al. [8] proposed a $2 \mathrm{~kW}$ full bridge PWM class D power amplifier for driving a 


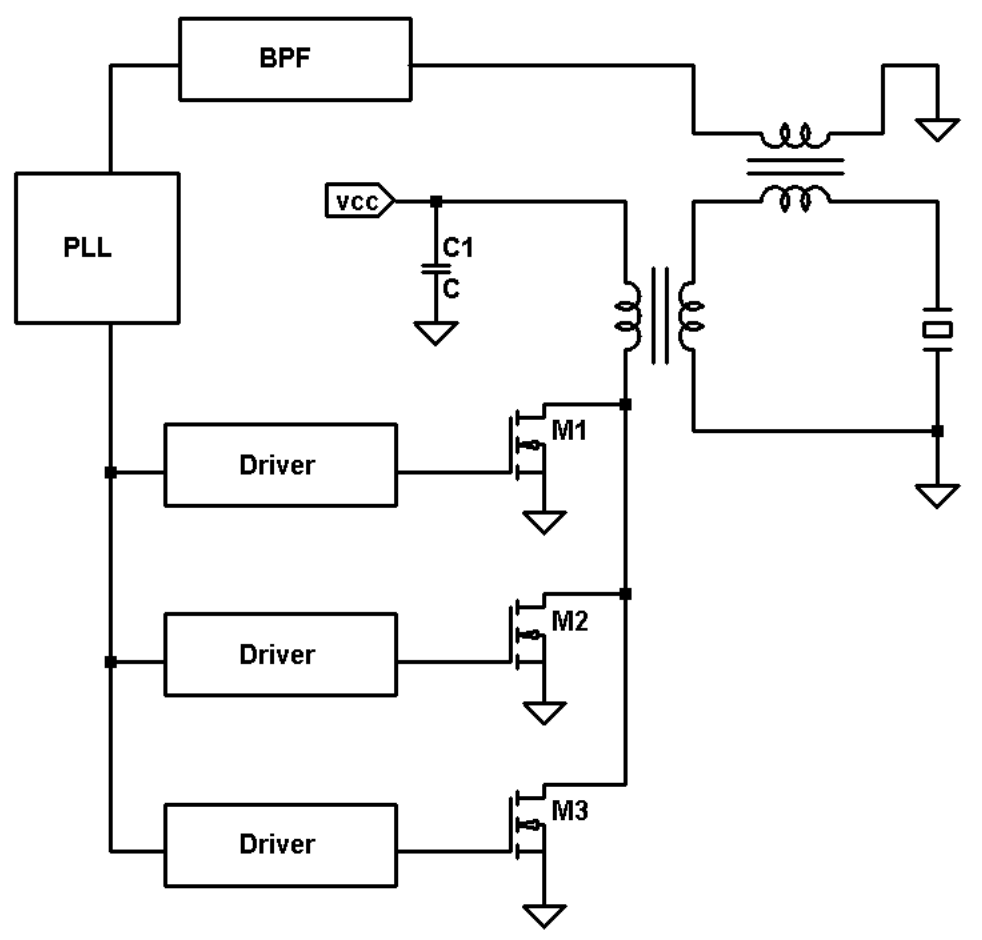

Figure 2.13: Schematic of DC to RF Inverter [7].

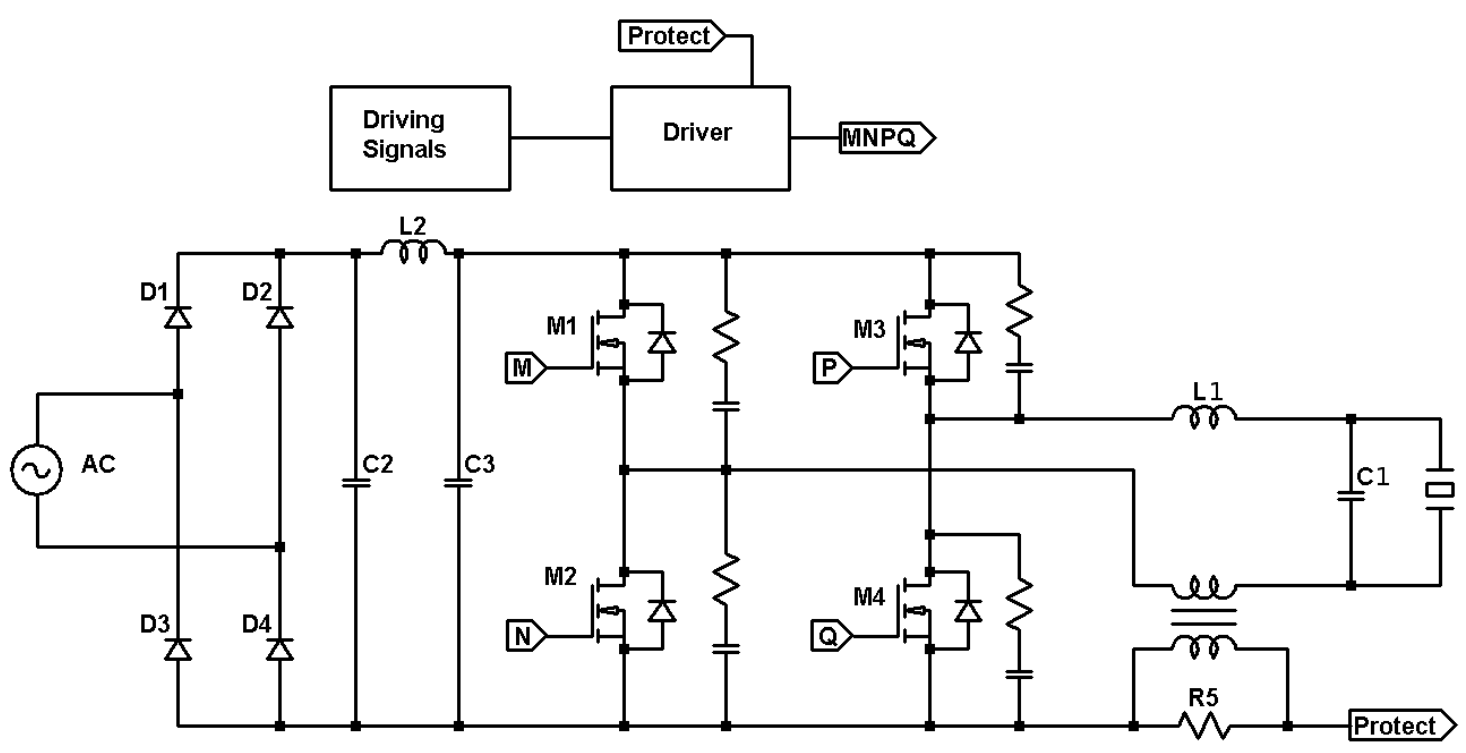

Figure 2.14: Schematic of class D inverter for piezoelectric transducer [8]. 


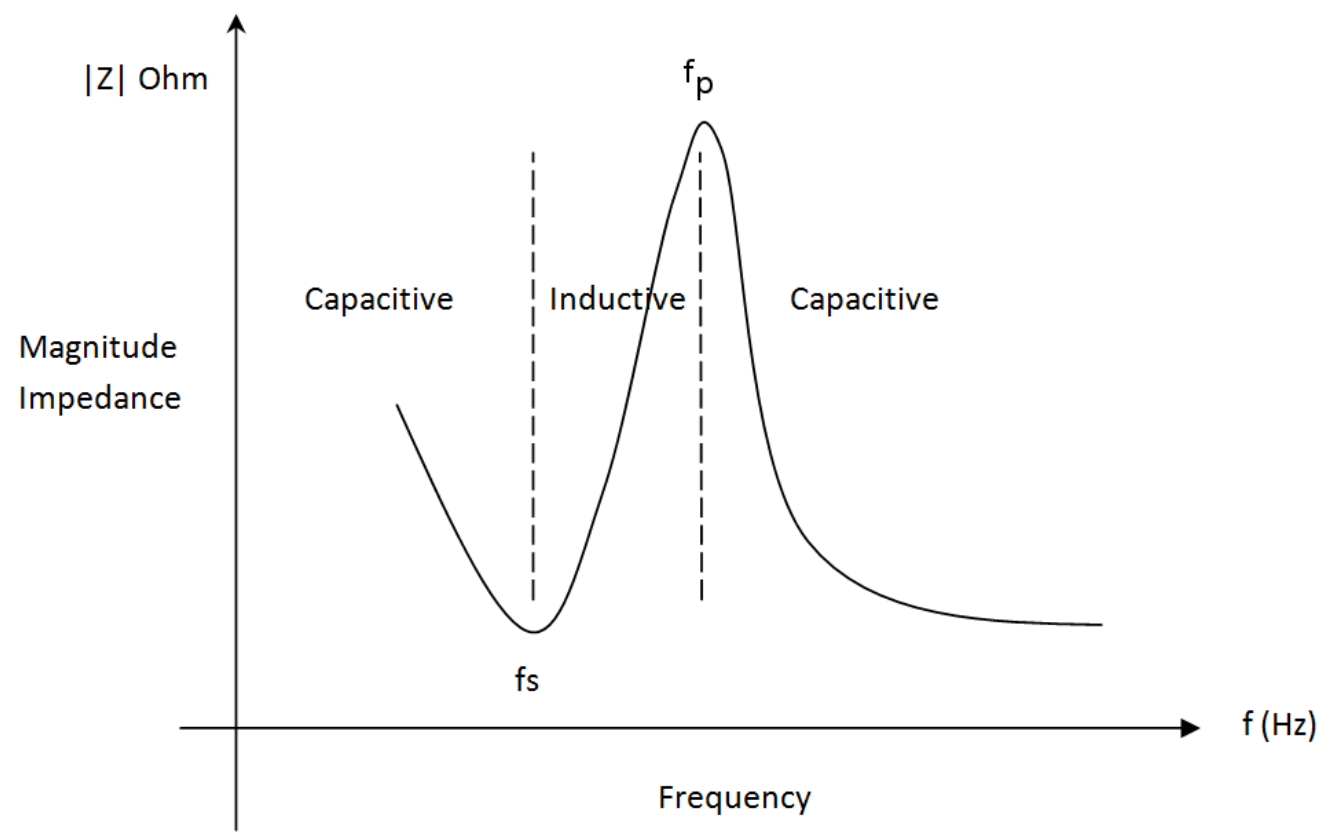

Figure 2.15: Magnitude impedance of piezoelectric ultrasound transducer close to one of its resonant frequencies. Variables $f_{s}$ and $f_{p}$ stand for series resonant frequency and parallel resonant frequency [8].

chemical reactor. The range of the operating frequency is between $10 \mathrm{kHz}$ and $100 \mathrm{kHz}$ and its efficiency is greater than $90 \%$. The topology of the proposed class D amplifier is shown in the Figure 2.14. A LPF ensures that the load impedance seen by the amplifier is either resistive or inductive.

The authors considered the impact on the amplifier performance due to the behaviour changes of the piezoelectric load when the operating temperature of the transducer is changing. Figure 2.15 shows the magnitude impedance of a piezoelectric transducer near one of its resonant frequency. If the operating frequency is equal to series resonant frequency, the impedance of the piezoelectric load is purely resistive. Hence, the acoustic output of the transducer is maximized because the phase shift between driving voltage waveform and current waveform is zero. Slight variations of temperature will change the thickness of the piezoelectric resonator as well as its behaviour. As shown in Figure 2.15, the impedance of a piezoelectric load is capacitive when its operating frequency is below resonant frequency $f_{s}$ or above parallel resonant frequency $f_{p}$. Similarly, the impedance of a piezoelectric load is inductive when its operating frequency is between $f_{s}$ and $f_{p}$. A RC snubber circuit and an anti-parallel diode are installed in parallel with 
the MOSFET drain and source terminal to protect the MOSFET. A $10 \mu \mathrm{F}$ capacitor across the bridge lessens the strength of the high frequency noise induced from the output to the power supply line.

A class D full-bridge amplifier with PWM is an excellent choice for low frequency and high power applications, usually below few hundred kilohertz. As mentioned in Section 2.2.2, class D full bridge amplifier is not suitable for our design.

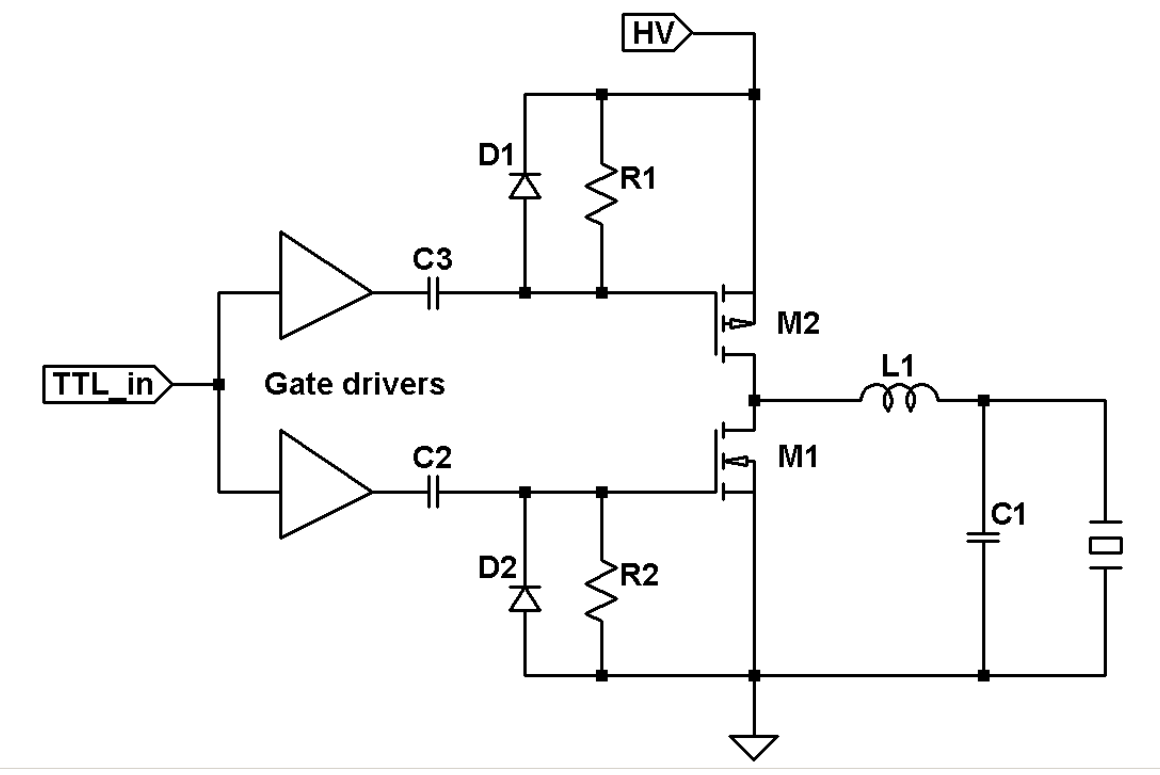

Figure 2.16: Schematic of switching amplifier and tuned filter [2].

Hall and Cain [2] proposed a 20 W, 1 MHz class D power amplifier for a 512-channel transducer array for transcutaneous ultrasound surgery. The efficiency of each amplifier is $90 \%$. Figure 2.16 is the schematic diagram of one channel.

The circuit of a switched amplifier, Figure 2.16, is similar to an inverter except MOSFETs are driven separately by their gate drivers. The on-off cycle of MOSFET M1 and M2 will still be determined by the TTL signal. As a result, the output signal is a square wave. Capacitors C1 and $\mathrm{C} 2$ and resistor R1 and R2 are responsible for level shifting for the gate signal because M2 is connected to high voltage. Tuned filter inductor L1 and capacitor C3 filter out higher harmonics in the square wave.

The advantage of this design is that it is simple and very cost effective. However, if the entire design has to be fabricated into a chipset, a resistor may occupy a very large portion of the area. 
Similarly, the inductor has to be off-chip.

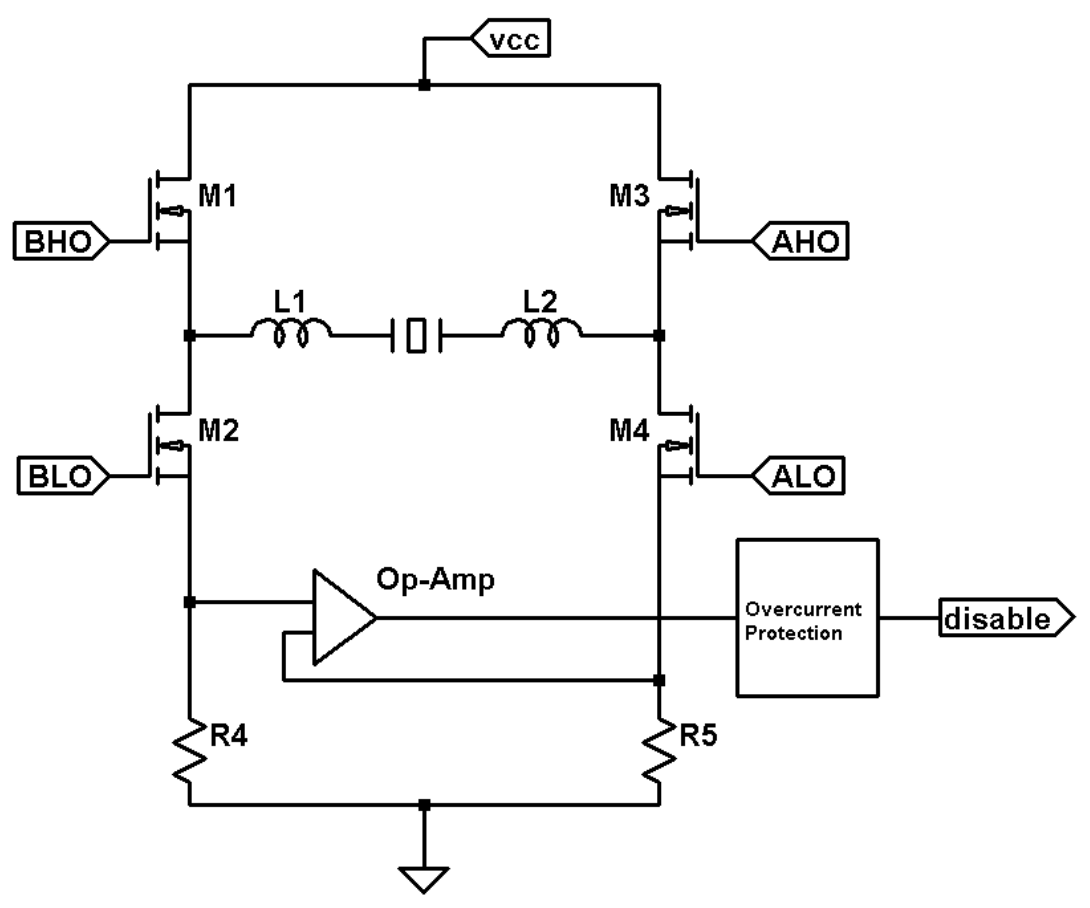

Figure 2.17: Schematic of class D amplifier for Audio Beam System [9].

Yang and Xu [9] proposed a class D full-bridge amplifier for an Audio Beam system. Unlike a magnetic speaker, an Audio Beam speaker is made with piezoelectric transducers; it can generate highly directional audio signals between $20 \mathrm{kHz}$ to $60 \mathrm{kHz}$. The switching frequency is $600 \mathrm{kHz}$. The schematic of its output stage is shown in Figure 2.17. Resistors R4 and R5 and an operational amplifier are part of the circuitry that cooperates with the gate driver IC to provide over-current protection to the MOSFETs at the output stage. Inductors L1 and L2 reduce the instantaneous current that goes to the transducer.

The output stage of this amplifier consists of four transistors and two inductors. It is not economical to implement our design with this topology because four transistors occupy more space and inductors have to be mounted externally.

Tang and Clement [10] evaluated the performance of the harmonic cancellation technique 


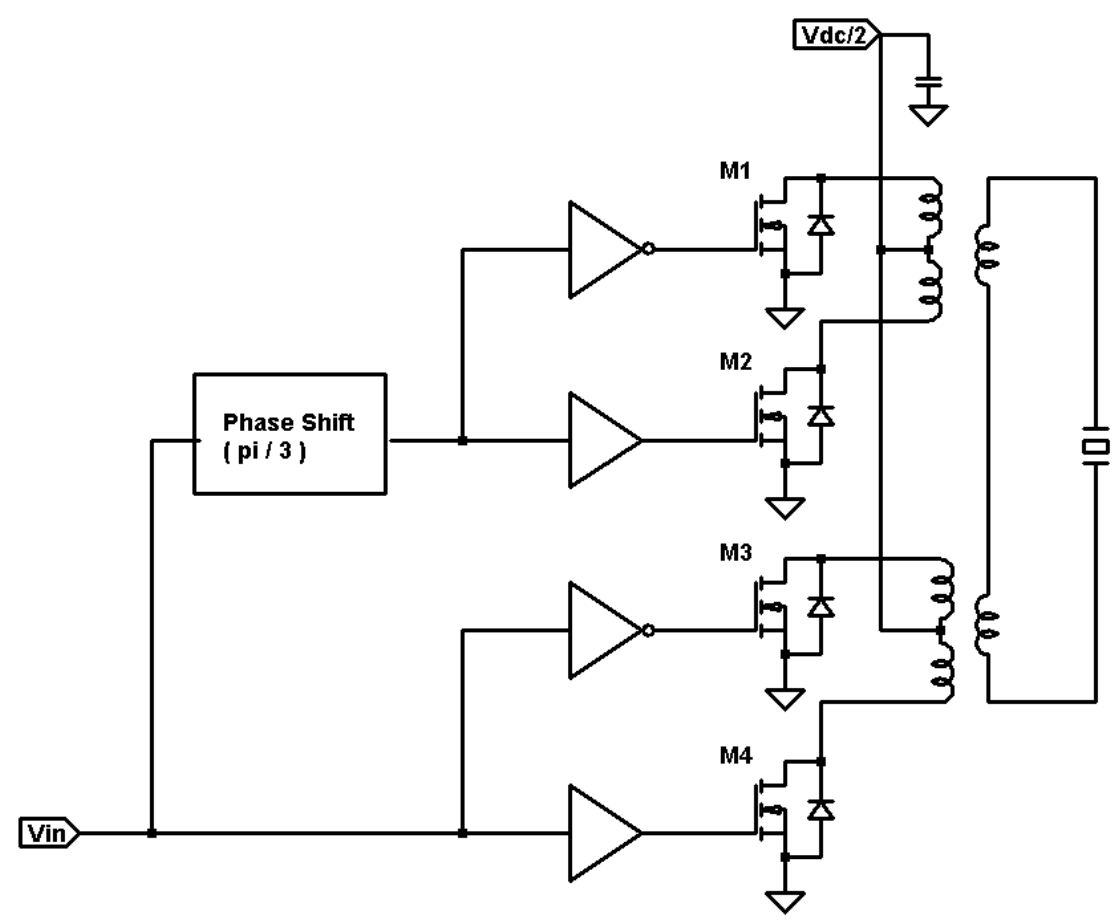

Figure 2.18: Schematic of power inverter for harmonic cancellation [10].

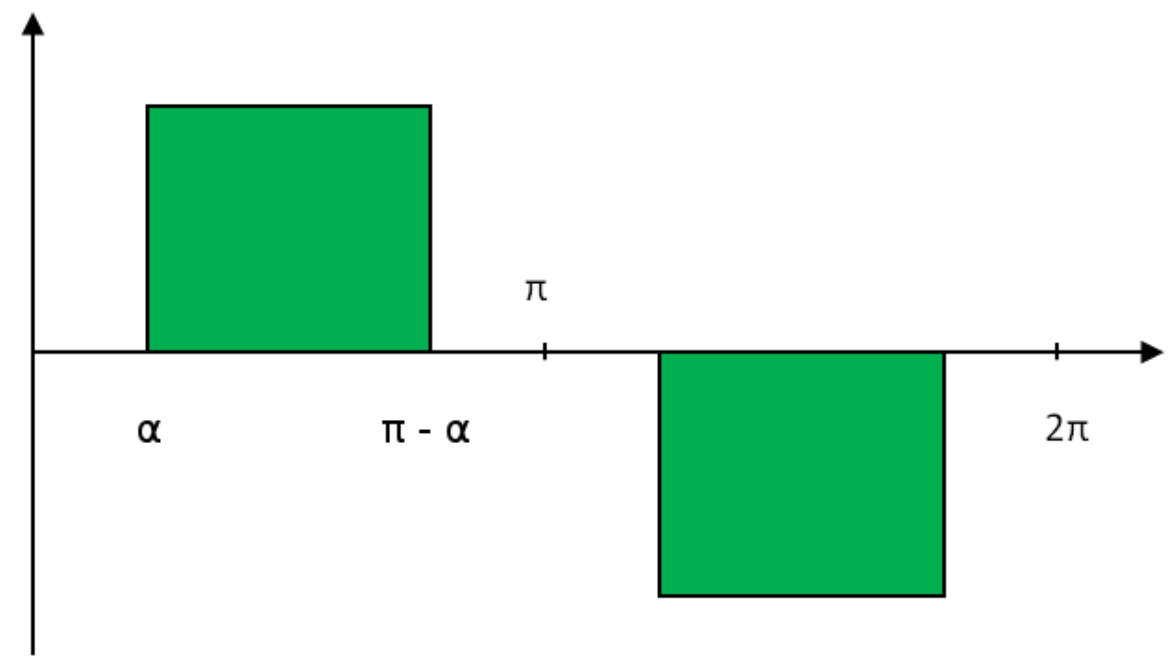

Figure 2.19: Waveform of harmonic cancellation. It eliminates the third harmonic if variable $a=\pi / 3$ $[10]$. 
for a therapeutic ultrasound transducer in HIFU applications. The schematic of the amplifier is shown in Figure 2.19. It comprises two power converters in cascade connection and the operating frequency is $1 \mathrm{MHz}$.

In this work, the authors pointed out that driving an ultrasound piezoelectric transducer with a signal that contains harmonics will distort the shape of the ultrasound focal spot. This is because harmonic contents present in electrical signals cause the transducer to generate unwanted sidelobes in the acoustic field. These sidelobes are carrying extra energy and will eventually distort the focal spot. The authors resolved this problem with the harmonic cancellation technique.

Harmonic cancellation is a technique that creates a square waveform with pre-calculated firing angles to suppress particular harmonics. As shown in Figure 2.18, a square waveform, with a firing angle of $\pi / 3$ used as driving signal, eliminates the 3rd harmonic. The converter does not require an additional LC filtering circuit; however, it employs two transformers. This technique successfully attenuates the $3 \mathrm{rd}$ harmonic from $-20 \mathrm{~dB}$ to $-48 \mathrm{~dB}$, comparing with the $-46 \mathrm{~dB}$ at the 5th harmonic. The mechanical characteristic of the transducer and electrical characteristics of the coax cable can attenuate higher harmonic contents naturally.

Harmonic cancellation waveform must be very precise. Rise time and fall time should be minimal; otherwise, unwanted harmonics will appear at the output. In addition, it is not practical to implement our design with this topology because the output stage comprises two transformers.

Cheng et al. [11] proposed a $41 \mathrm{kHz}, 42 \mathrm{~W}$ single switch class E amplifier with power factor correction as an ultrasonic cleaner. The overall efficiency of the inverter is $90 \%$. As shown in Figure 2.20, the circuit comprises an AC power regulator, flyback and class E amplifier. The class E amplifier and the flyback stage share only one switch. The output of the class E amplifier feeds a resonant circuit and a transducer.

As mentioned earlier in the section of the class $\mathrm{E}$ amplifier, choke RFC is an inductor with very high inductance. It is too big for our design. Therefore, this topology is not suitable for the integrated amplifier.

Lewis and Olbricht [12] developed a 48 W, $1.54 \mathrm{MHz}$ high intensity ultrasound system for military, medical and research applications. The efficiency of the amplifier is $99 \%$ as reported by 


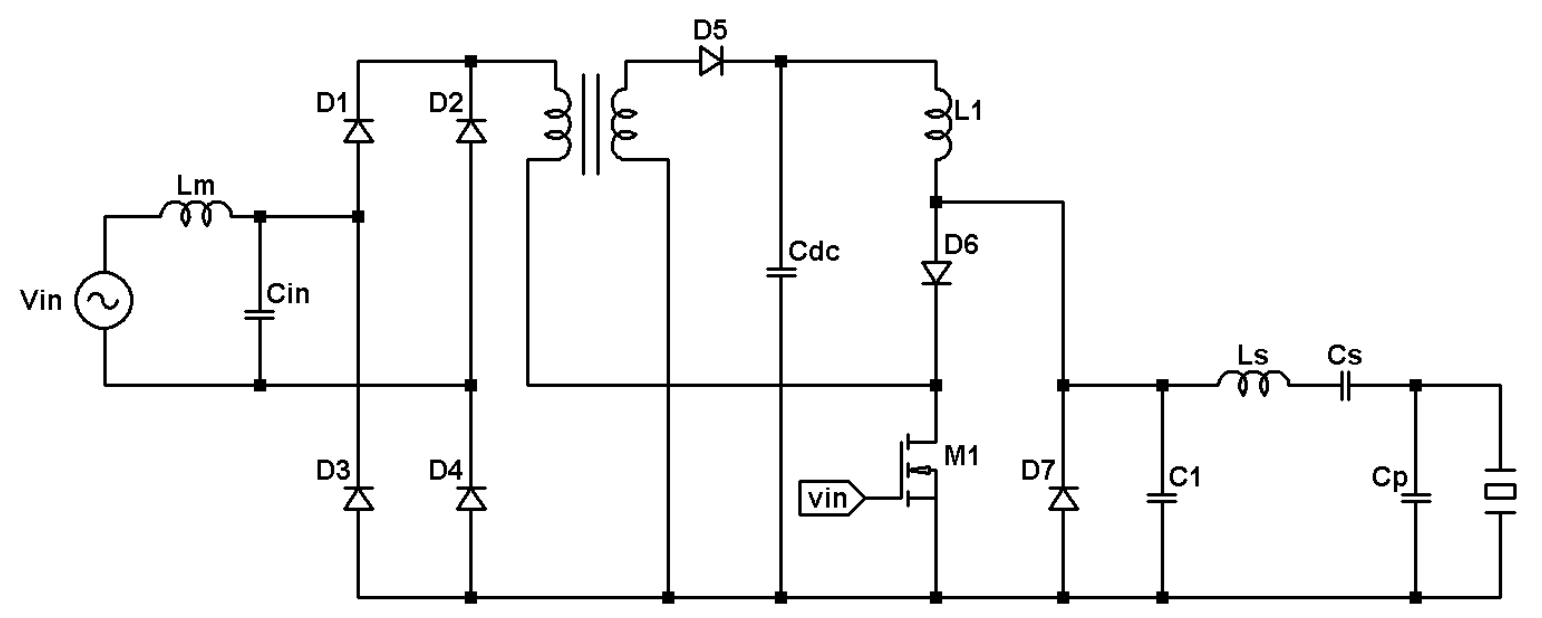

Figure 2.20: Schematic of class E amplifier with PFC [11].

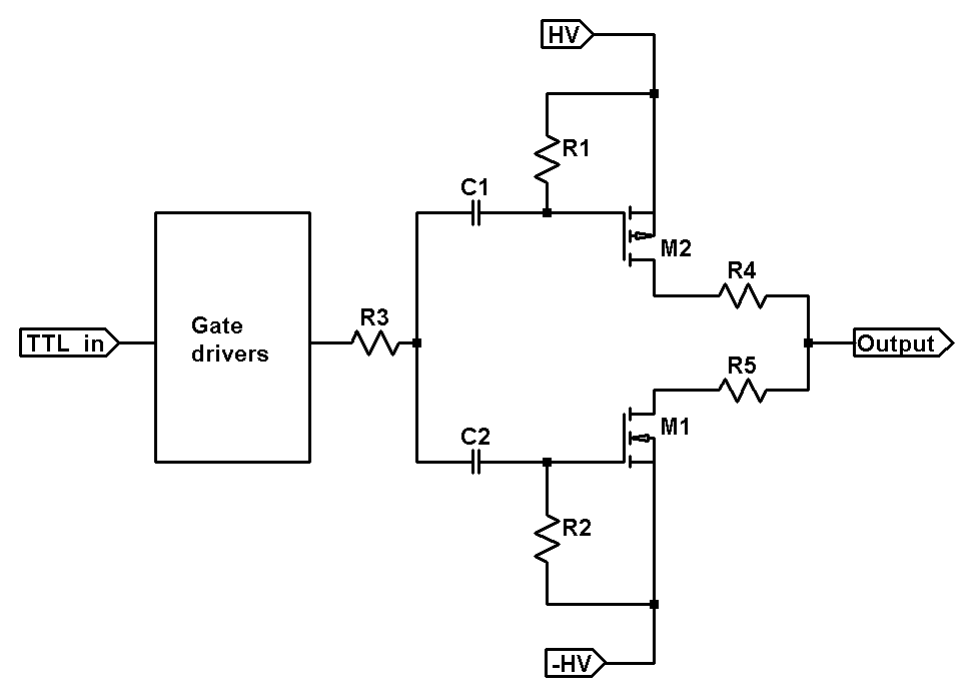

Figure 2.21: Schematic of switched-mode amplifier [12]. 
the authors. Its schematic is shown in Figure 2.21. In order to achieve maximum power transfer from a standard $50 \mathrm{Ohm}$ power supply to a $50 \mathrm{Ohm}$ transducer probe, the output impedance of the amplifier must be as low as possible. The authors pointed out if $99 \%$ of the voltage from the power supply must be transfered to load, the output impedance of the power amplifier should not be greater than $0.05 \mathrm{Ohm}$. Their design was implemented using discrete components. Multiple power transistors are connected in parallel with each other to achieve very low output impedance. A RC network handles level shifting for MOSFETs. The overall design is lightweight, small in size and cost effective. [12] Its topology is similar to [2] without a tuned LC harmonic filter connecting between the amplifier output and transducer.

Since the driver is a class D amplifier, the output waveform of the amplifier is a square wave. Driving an ultrasound transducer with a square wave will cause the acoustic pressure field to contain harmonics; therefore this solution cannot be adapted to HIFU applications directly.

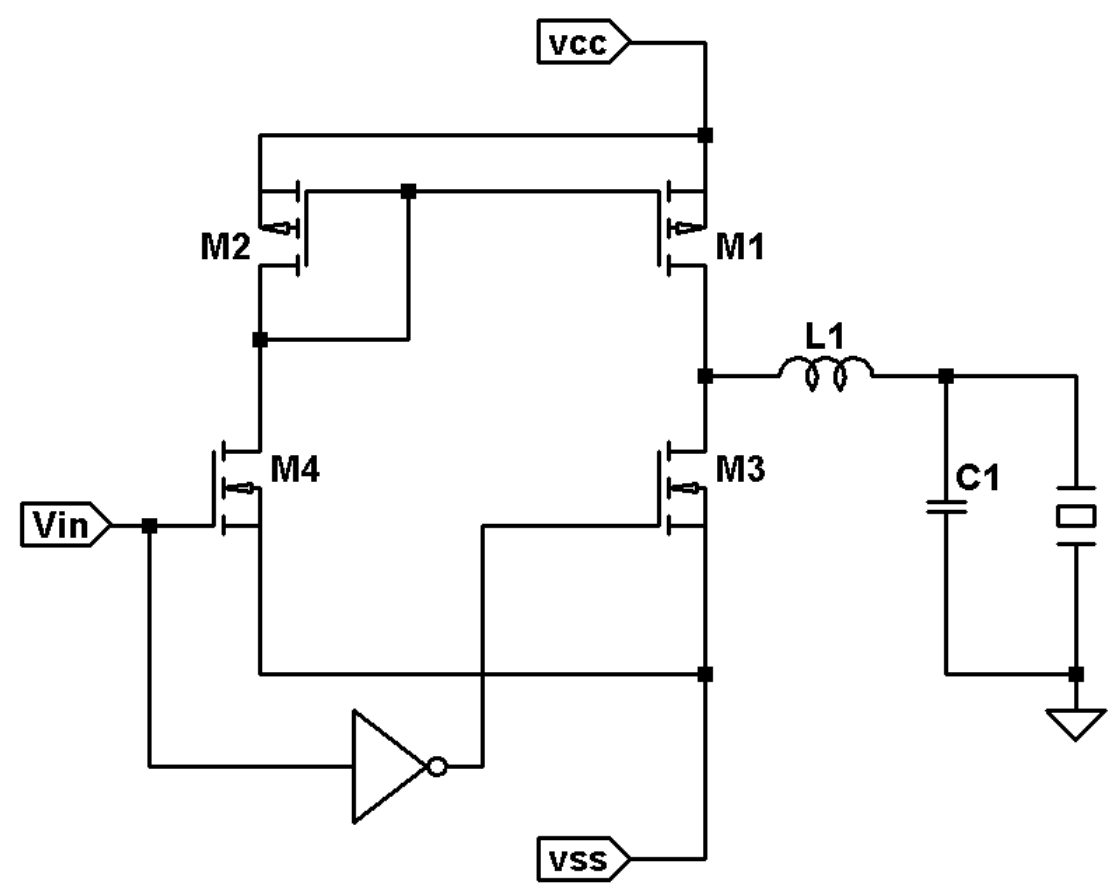

Figure 2.22: Schematic of output stage and matching network of LIPUS amplifier [13].

Low Intensity Pulsed Ultrasound, LIPUS, is an emerging technique that uses ultrasound for 
bone healing, dental tissue formation, and tooth-root healing. Ang et al. [13] had fabricated a $0.8 \mathrm{~W}, 1.5 \mathrm{MHz}$ integrated amplifier chip for LIPUS applications. Its schematic is shown in Figure 2.22 and it was fabricated with DALSA $0.8 \mu \mathrm{m}$ HV CMOS process. The overall efficiency including a pulse generator and an amplifier is $70 \%$.

Major challenges of LIPUS devices are portability and ultrasound transducer impedance matching. First, the power amplifier and its signal generator are too large to fit for orthodontic treatment. With the aid of advanced CMOS technologies, the authors successfully scaled down both the pulse generator stage and the power output stage to a $2.8 \mathrm{~mm} \times 4 \mathrm{~mm}$ chip. Second, depending on the impedance characteristics of the piezoelectric resonator, an ultrasound transducer usually requires high voltage and high current to produce enough acoustic power for healing purposes. In order to mitigate the electrical requirements, an external tuned LC matching network that matches impedance of the transducer at resonance frequency is inserted in between the output of amplifier and the transducer. This LC filter boosts the output voltage form $2.53 \mathrm{~V}$ peak to $7.6 \mathrm{~V}$ peak and eliminates higher harmonic contents from the output signals. A level shifter was used as the power output stage as shown in Figure 2.22. The generation of pulse modulated signals is done by a digital block.

Although this device was implemented as integrated circuit, it occupies an area of $2.8 \mathrm{~mm} \times$ $4 \mathrm{~mm}$ and employs a matching network. In addition, in our case, an efficiency of $70 \%$ may create thermal problems.

Table 2.2 summarizes the specifications and comments of all published works studied in this section.

\subsection{Summary}

Although published works provide valuable information and techniques for designing piezoelectric amplifiers, none of the published works offers an immediate solution to resolve our challenges, such as elimination of inductors. Hence, another approach is needed. Tables 2.1 and 2.2 are compendia of this chapter. 
Table 2.1: Comparison of amplifier topologies.

\begin{tabular}{|c|c|c|c|c|}
\hline Topologies & Waveforms & $\begin{array}{l}\text { Theoretical } \\
\text { max. efficiency }\end{array}$ & $\begin{array}{l}\text { Harmonic } \\
\text { contents }\end{array}$ & $\begin{array}{l}\text { Comments for } \\
\text { implementations }\end{array}$ \\
\hline [4] Class A & Sinusoidal & $50 \%$ & Low & $\begin{array}{l}\text { Highly linear, } \\
\text { thermal problem }\end{array}$ \\
\hline [4] Class B & $\begin{array}{l}\text { Positive half } \\
\text { of the sinusoidal }\end{array}$ & $78.5 \%$ & High & Unsuitable, needs a tuned network \\
\hline [4] Class AB & $\begin{array}{l}\text { More than half } \\
\text { of the sinusoidal }\end{array}$ & $78.5 \%$ & Intermediate & $\begin{array}{l}\text { Acceptable, needs a tuned network. } \\
\text { High efficiency configuration eases } \\
\text { the thermal problem. }\end{array}$ \\
\hline [4] Class D & Square wave & $100 \%$ & High & $\begin{array}{l}\text { Cannot apply to transducer directly. } \\
\text { Needs a tuned network. }\end{array}$ \\
\hline [4] Class D PWM & PWM & $100 \%$ & High & $\begin{array}{l}\text { Maximum freq. is limited by } \\
\text { technologies; needs LPF. }\end{array}$ \\
\hline [4] Class E & Sinusoidal & $100 \%$ & High & $\begin{array}{l}\text { Needs choke (oversize); } \\
\text { requires a tuned network. }\end{array}$ \\
\hline [5] Class DE & $\begin{array}{l}\frac{1}{4} T \text { of sinusoidal } \\
\text { and } \frac{1}{4} T \text { of level hold }\end{array}$ & $100 \%$ & High & $\begin{array}{l}\text { Non-linear, } \\
\text { requires a tuned network. }\end{array}$ \\
\hline [6] Step-up & Square wave & $100 \%$ & High & $\begin{array}{l}\text { Needs inductor } \\
\text { three switches. }\end{array}$ \\
\hline [6] Flyback & Sinusoidal & & Low & Needs a transformer. \\
\hline [6] Push-pull & Sinusoidal & & Low & Needs a transformer. \\
\hline
\end{tabular}


Table 2.2: Comparison of specifications of published works.

\begin{tabular}{|c|c|c|c|c|c|c|c|}
\hline References & $\begin{array}{l}\text { Operating } \\
\text { frequencies }\end{array}$ & $\begin{array}{l}\text { Measured } \\
\text { efficiency }\end{array}$ & Topologies & $\begin{array}{l}\text { Output } \\
\text { power }\end{array}$ & Excitation & Applications & Comments \\
\hline$[7]$ & $1 \mathrm{MHz}$ & & Flyback & $60 \mathrm{~W}$ & Sinusoidal & $\begin{array}{l}\text { Wafer } \\
\text { Cleaning }\end{array}$ & A transformer is needed. \\
\hline$[8]$ & $10-100 \mathrm{kHz}$ & $95 \%$ & $\begin{array}{l}\text { Class D } \\
\text { full bridge }\end{array}$ & $2 \mathrm{~kW}$ & PWM & $\begin{array}{l}\text { Chemical } \\
\text { Mixing }\end{array}$ & Needs an LC network. \\
\hline$[2]$ & $1 \mathrm{MHz}$ & $>90 \%$ & $\begin{array}{c}\text { Class D } \\
\text { half bridge }\end{array}$ & $20 \mathbf{W}$ & sinusoidal & $\begin{array}{l}\text { Ultrasound } \\
\text { Therapy }\end{array}$ & Needs an LC network. \\
\hline$[9]$ & $20-60 \mathrm{kHz}$ & & $\begin{array}{l}\text { Class D } \\
\text { full bridge }\end{array}$ & & PWM & Audio & $\begin{array}{l}\text { Needs inductors and four } \\
\text { transistors (more area). }\end{array}$ \\
\hline [10] & $1 \mathrm{MHz}$ & & $\begin{array}{c}\text { Class D } \\
\text { half bridge }\end{array}$ & & sinusoidal & $\begin{array}{l}\text { Ultrasound } \\
\text { Therapy }\end{array}$ & A transformer is needed \\
\hline$[11]$ & $41 \mathrm{kHz}$ & $93 \%$ & Class E & $42 \mathbf{W}$ & sinusoidal & $\begin{array}{l}\text { Ultrasonic } \\
\text { Cleaning }\end{array}$ & Needs an LC network. \\
\hline$[12]$ & 1 to $10 \mathrm{MHz}$ & $90 \%$ & $\begin{array}{c}\text { Class D } \\
\text { half bridge }\end{array}$ & $50 \mathrm{~W}$ & $\begin{array}{l}\text { square } \\
\text { wave }\end{array}$ & $\begin{array}{l}\text { Ultrasound } \\
\text { Therapy }\end{array}$ & $\begin{array}{l}\text { Output waveforms } \\
\text { are square waves. }\end{array}$ \\
\hline$[13]$ & $1.5 \mathrm{MHz}$ & $70 \%$ & $\begin{array}{c}\text { Class D } \\
\text { half bridge }\end{array}$ & $0.58 \mathrm{~W}$ & sinusoidal & $\begin{array}{l}\text { Ultrasound } \\
\text { Therapy }\end{array}$ & Needs an LC network. \\
\hline
\end{tabular}




\section{Chapter 3}

\section{Characterisation of an Ultrasound}

\section{Transducer}

\section{$3.1 \quad$ Transducer}

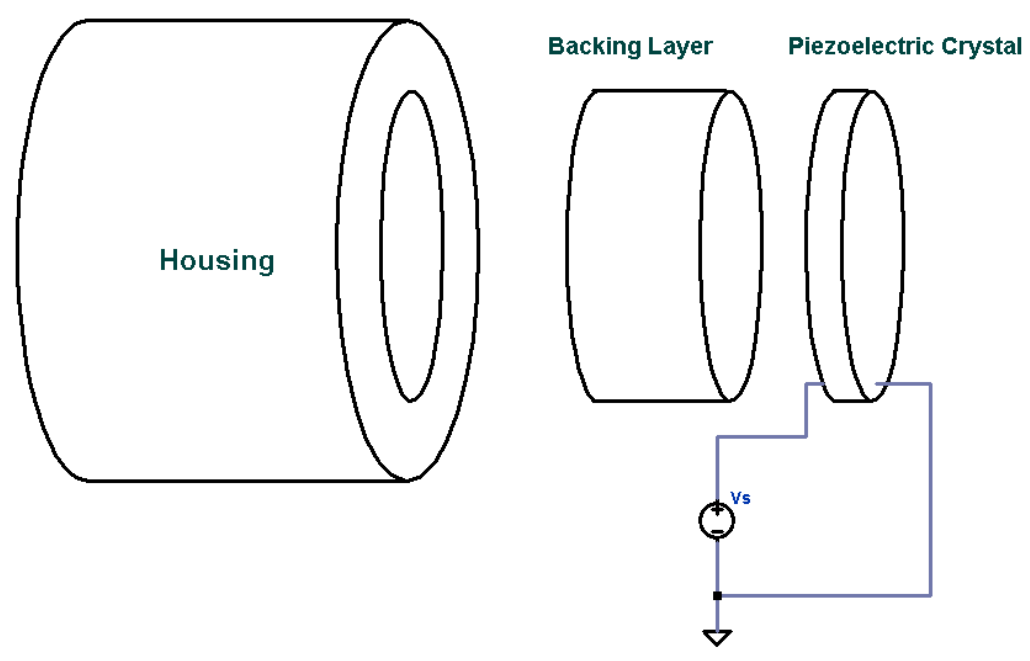

Figure 3.1: Exploded view of a transducer.

Ultrasound can be generated through a simple device called a transducer. Figure 3.1 shows an exploded view of a therapy transducer [14]. The rightmost component is a piezoelectric resonator that generates ultrasound. The schematic diagram of housing and assembly instructions of the 
ultrasound transducer can be found in Appendix A. For therapy transducers, the material of this resonator is commonly made of Lead Zirconate Titanate, PZT. Electrodes are coated on both sides of the resonator for electrical connections. The outer case is called the housing. It protects both the internal circuitry and the piezoelectric resonator from the physical environment and provides air backing for the resonator. Circuitry, such as a tuned filter and matching network, can be mounted internally. A coax cable is used for signal transmission from the amplifier to the piezoelectric resonator.

\subsection{Equivalent Circuit}

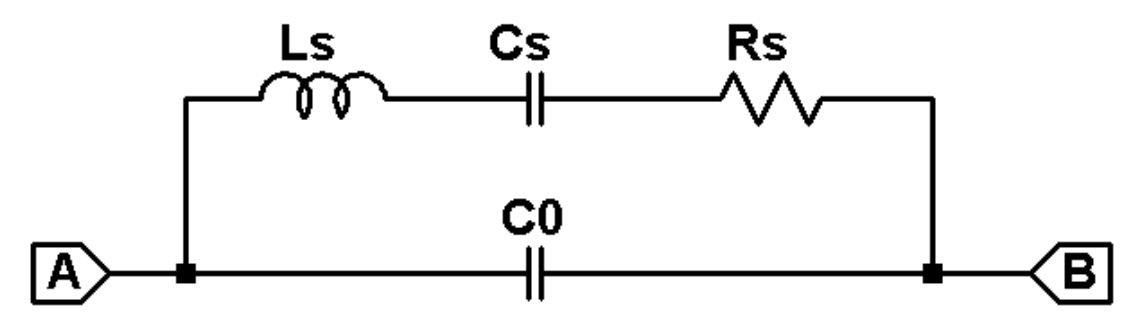

Figure 3.2: Equivalent circuit of a piezoelectric resonator near its resonance frequency [15].

The equivalent circuit of a piezoelectric resonator vibrating near its resonance is shown in Figure 3.2 [15]. It comprises a capacitor connected in parallel with a series resonant circuit. Mathematically, the impedance of equivalent circuit of Figure 3.2 can be expressed as follows:

$$
Z=\frac{\frac{1}{j \omega C_{0}}\left(\frac{1}{j \omega C_{S}}+R_{S}+j \omega L_{S}\right)}{\frac{1}{j \omega C_{0}}+\frac{1}{j \omega C_{S}}+R_{S}+j \omega L_{S}}
$$

where $\omega$ is angular frequency; symbol $\mathrm{j}$ denotes $\sqrt{-1}$. The variable $C_{0}$ is static capacitance that represents the electrical branch of the resonator. It is determined by the physical properties of the resonator, such as the permittivity with zero strain, surface area of the piezoelectric resonator, and the distance between electrodes. The quantities $C_{s}, L_{s}$ and $R_{s}$ denote the series resonant circuit that stands for the mechanical branch of the resonator, and $R_{s}$ includes both the mechanical losses and the mechanical power transferred to acoustic field. Terminals A and $\mathrm{B}$ are inputs of the equivalent circuit. Usually, one of them is named HOT, and connects to the output of amplifier or function generator. The other terminal is called Ground, which connects to the earth or reference point of circuit. 


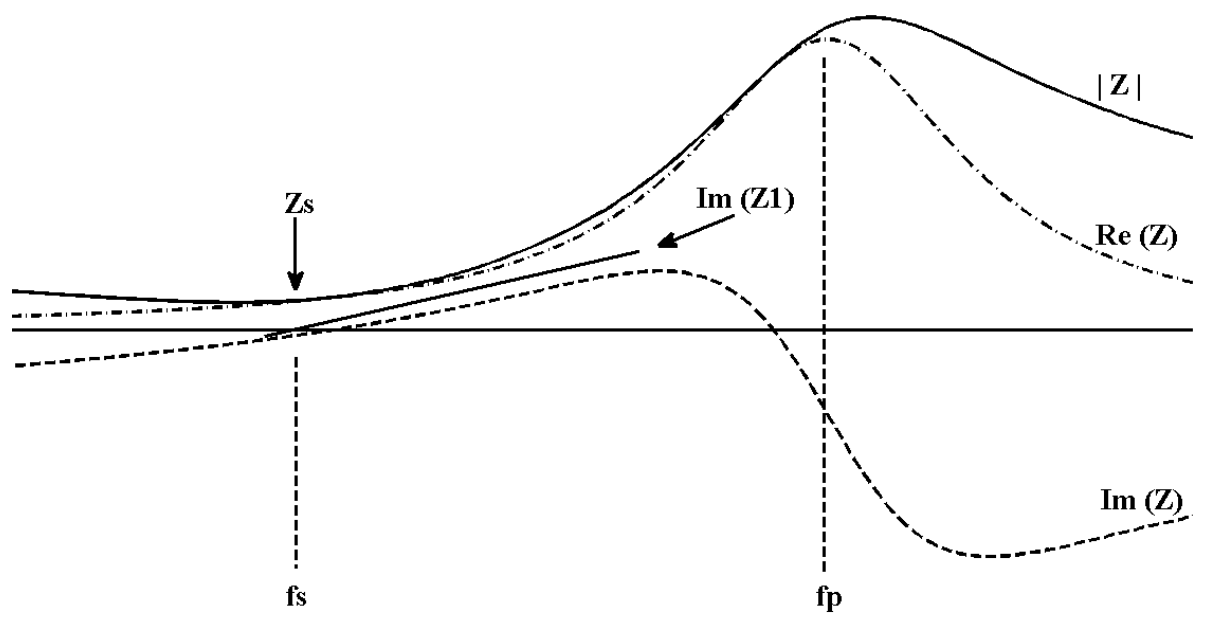

Figure 3.3: Impedance of equivalent circuit in Figure 3.2 [15].

The impedance of the equivalent circuit as a function of frequency is shown in Figure 3.3 [15]. The magnitude impedance of the equivalent circuit is denoted by $|Z|$, and $\operatorname{Re}(Z)$ and $\operatorname{Im}(Z)$ are the real and imaginary components of the impedance $\mathrm{Z} ; f_{p}$ is parallel resonant frequency in which the real part of the impedance $\mathrm{Z}$ reaches maximum; and $f_{s}$ is the series resonant frequency when the reactance of the series resonant circuit $\operatorname{Im}(Z 1)$ is zero, where $Z 1=R_{s}+j \omega L_{s}+\frac{1}{j \omega C_{s}}$. When the equivalent circuit is operating at the resonant frequency, the reactance of $L_{s}$ and $C_{s}$ cancel out each other, leaving only $C_{0}$ parallel with $R_{s}$. For this reason, it is common to choose an excitation frequency very close to the series resonant frequency, $f_{s}$, as the operating frequency, because the voltage can transfer to $R_{s}$ directly. Therefore, the supply voltage will be minimal.

\subsection{Mathematical Model}

At series resonant frequency $f_{s}$, the reactance of $L_{s}$ and $C_{s}$ cancel out each other. We simplify Equation (3.1).

$$
\left.Z_{s}\right|_{\omega=\omega_{s}}=\frac{\frac{R_{s}}{j \omega_{s} C_{0}}}{\frac{1}{j \omega_{s} C_{0}}+R_{s}}
$$

Formulae for $C_{s}$ and $L_{s}$ are provided in [15]:

$$
\begin{gathered}
C_{s}=C_{0}\left[\left(\frac{f_{p}}{f_{s}}\right)^{2}-1\right] \\
L_{s}=\frac{1}{\left(2 \pi f_{s}\right)^{2} C_{s}}
\end{gathered}
$$


Solving for $C_{0}$ and $R_{s}$ :

$$
\begin{gathered}
C_{0}=\frac{-\operatorname{Im}\left(Z_{s}\right)}{2 \pi f_{s}\left|Z_{s}\right|^{2}} \\
R_{S}=\frac{\left|Z_{s}\right|^{2}}{\operatorname{Re}\left(Z_{s}\right)}
\end{gathered}
$$

Equations 3.3 to 3.4 are applied at the fundamental frequency to calculate $C_{0}, C_{s 1}, L_{s 1}$ and $R_{s 1}$. Once $C_{0}$ is found; apply equations 3.4, 3.6 and 3.3 to find the LRC values for all remaining odd harmonic branches. By connecting all odd harmonic branches with the fundamental branch in parallel as shown in Figure 3.4 [14], an equivalent circuit of a multi-mode piezoelectric resonator is found.

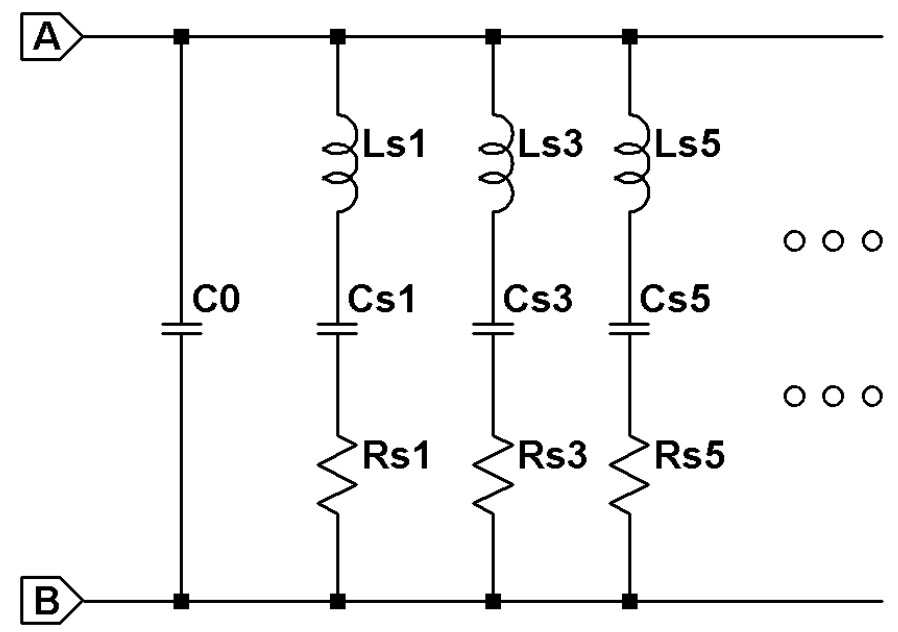

Figure 3.4: Equivalent circuit of a transducer [14].

The odd harmonics are a set of resonant frequencies based on the odd multiples of the fundamental resonant frequency. They depend on the thickness and physical properties of the piezoelectric material [14]. As shown in Figure 3.4 [14], the variable $C_{0}$ denotes the static capacitor. Components $L_{s 1}, C_{s 1}$, and $R_{s 1}$ comprise the fundamental branch. Similarly, $L_{s 3}, C_{s 3}$, and $R_{s 3}$ represent the third harmonic branch, and so on.

In practice, however, more resonant frequencies can be found because a piezoelectric resonator is a three dimensional object. These non-harmonic frequencies do not correspond to harmonics or multiples of the fundamental frequency [14]. 


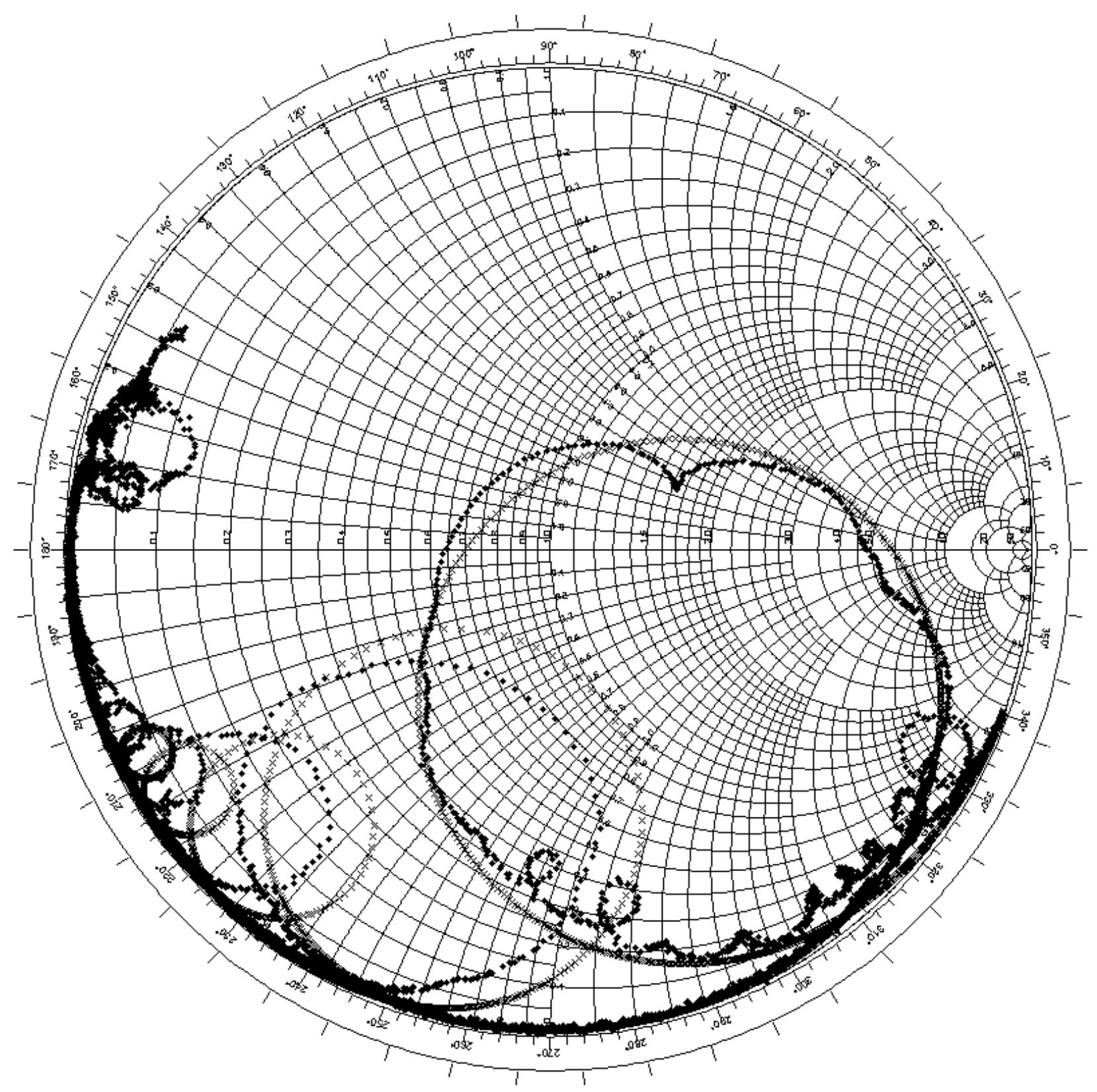

Figure 3.5: Comparison of the impedance of ultrasound transducer and equivalent circuit on a Smith Chart from $300 \mathrm{kHz}$ to $300 \mathrm{MHz}$. 


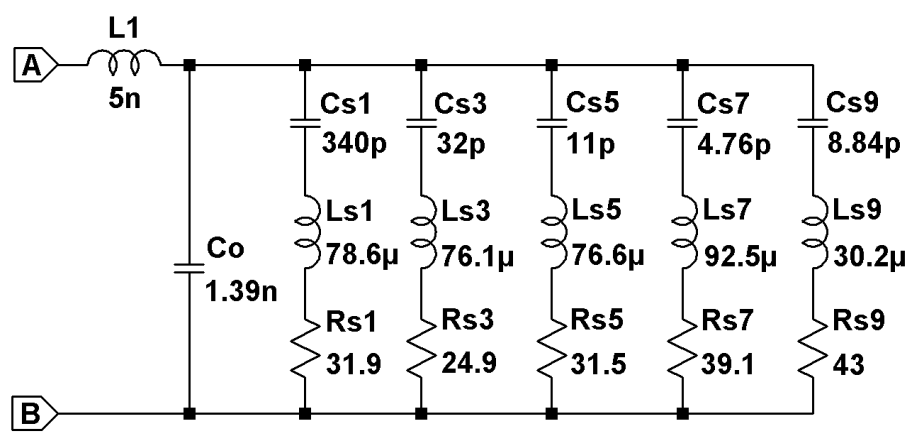

Figure 3.6: Equivalent circuit of the piezoelectric resonator up to the 9th harmonic.

\subsection{Results}

An experiment was preformed to measure the impedance of a piezoelectric resonator using a Vector Network Analyzer (VNA). A single element ultrasound transducer was made for this purpose. A calibration procedure was used to remove the parasitic effects induced by coax cables and Bayonet Neill- Concelman (BNC) connectors. Figure 3.5 shows the impedance characteristics of the piezoelectric resonator on the Smith chart. Each of the several resonance loops, up to the 9th harmonic observed in that figure, is modelled with an independent RLC branch in the equivalent circuit model. Figure 3.6 shows the equivalent circuit of the piezoelectric resonator. It behaves inductively at high frequency mainly due to the inductance of the three short conducting wires connected to it. Figure 3.7, transducer impedance as a function of frequency, compares the equivalent circuit with the measured data. 


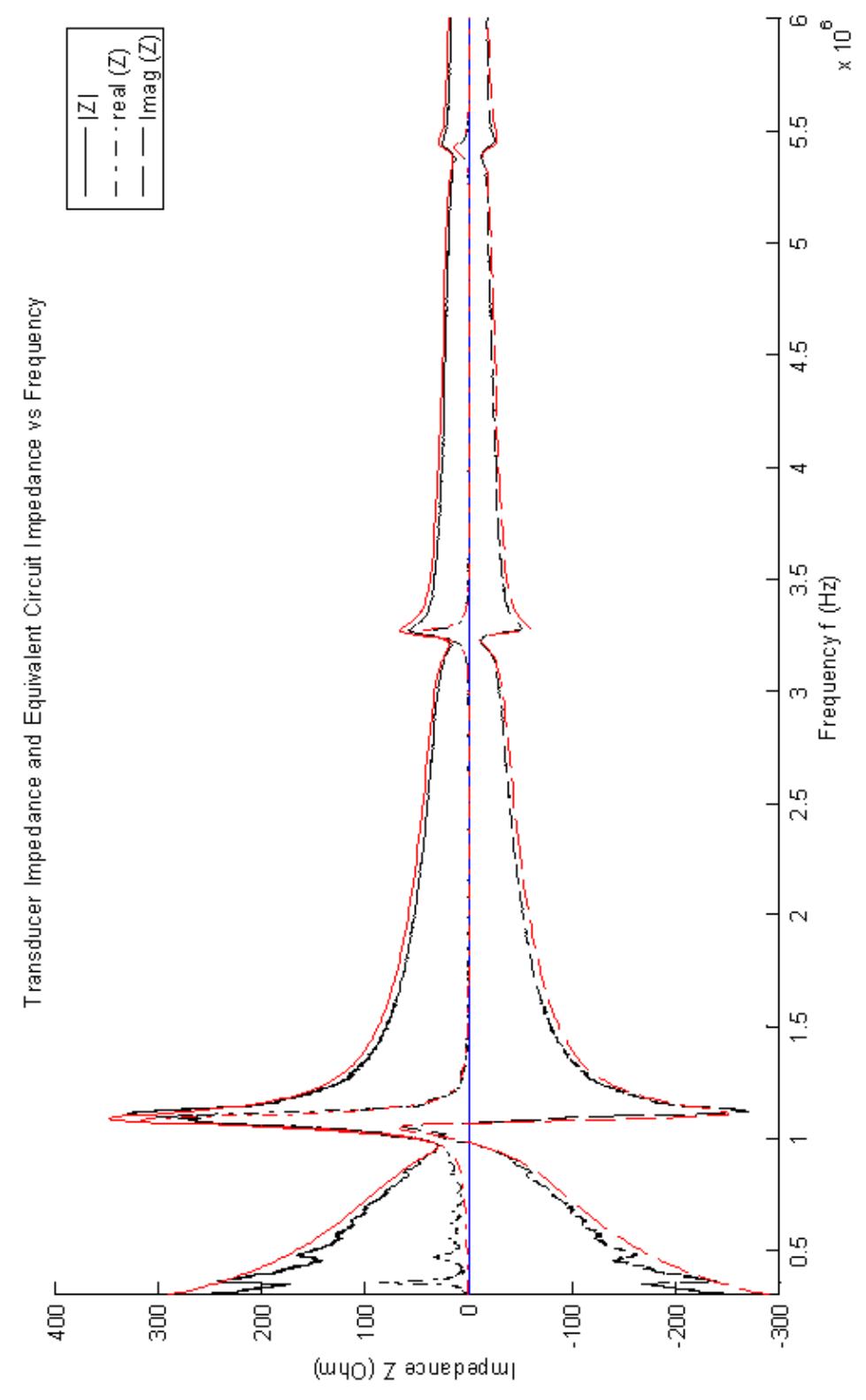

Figure 3.7: Comparison of the impedance of equivalent circuit and the measured results from VNA between $300 \mathrm{kHz}$ and $6 \mathrm{MHz}$, red - simulation of equivalent circuit, black - measured results. 


\subsection{Ultrasound Field Characterization}

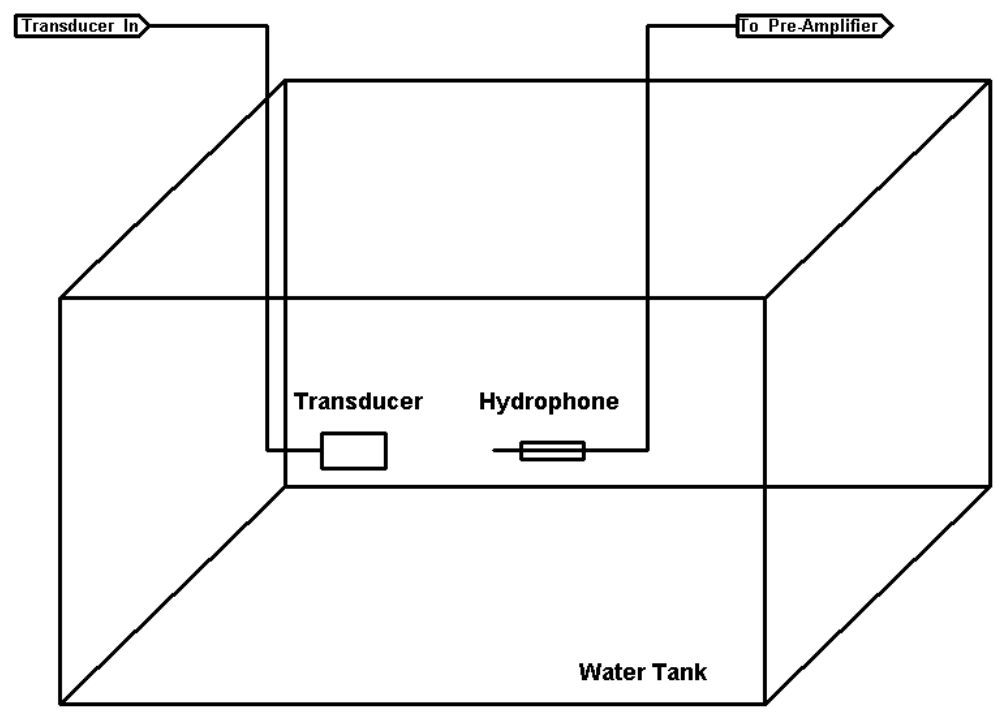

Figure 3.8: Schematic diagram of the ultrasound field characterization using three-dimensional positing system [14].

This section investigates the relation of the ratio of the electric fundamental to 3rd harmonic and mechanical fundamental to 3rd harmonic. The mechanical attenuation between fundamental and third harmonic are different. The goal is to predict the ratio of fundamental and 3rd harmonic in ultrasound field with given electrical signals, such as the output signals of an amplifier.

The intensity of the ultrasound field can be measured through a wave field scan with a three-dimensional positioning system. Figure 3.8 is a schematic of this system [14]. As shown in the Figure 3.8, a power amplifier sends an RF signal to the transducer. On the right, a small needle-like device called a hydrophone reads the acoustic pressure in front of the transducer. The received signal is amplified by a pre-amplifier. A personal computer uses with the signal generator and oscilloscope to record the position of the hydrophone, transmitted signals and received signals. The water tank is filled with degassed and de-ionized water. 


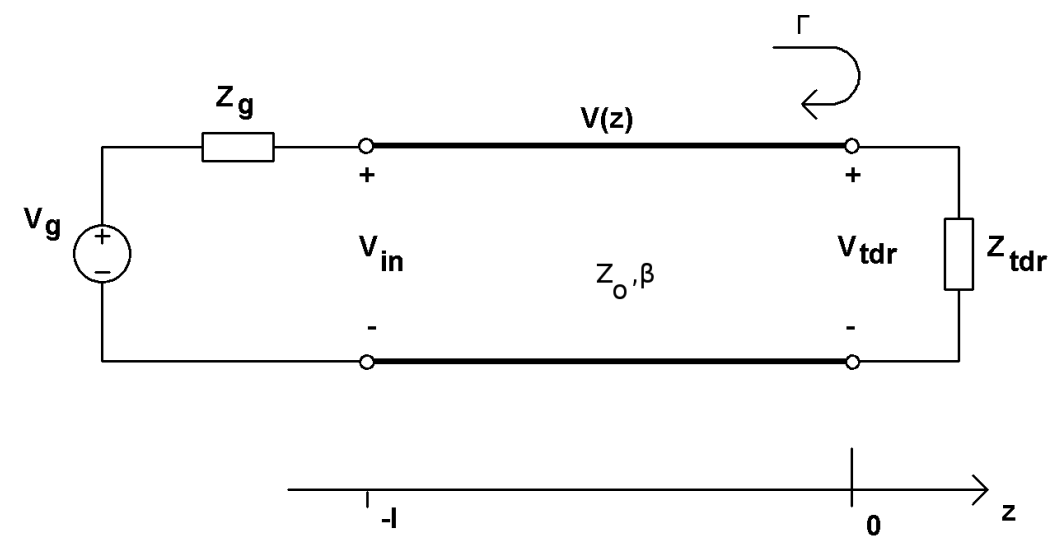

Figure 3.9: Schematic diagram of a voltage source driving a transducer through a coax [16].

\subsection{Calculations}

Our goal is to derive an expression for the the voltage $V_{t d r}$ that is applied to the piezoelectric resonator. As shown in Figure 3.9 [16], RF signals are transmitted from the function generator through a transmission line to the transducer. Variable $l$ denotes the distance from the load; voltage source $V_{g}$ stands for the function generator; $Z_{g}$ denotes output impedance of the generator; $\Gamma$ is the reflective coefficient of the transmission line; $Z_{t d r}$ represents the impedance of the transducer. $Z_{o}$ and $\beta$ stand for characteristic impedance and propagation constant respectively, of the transmission line [16]. Beginning with the expression of voltage $V(z)$ along a transmission line, we have [16],

$$
V(z)=V^{+}\left(e^{-j \beta z}+\Gamma e^{j \beta z}\right)
$$

where $V(z)$ is the instantaneous voltage at any point along $z$-axis. Variable $\beta$ is the propagation constant of a transmission line, which is defined as

$$
\beta=j \omega \sqrt{L_{T L} C_{T L}}
$$

where $L_{T L}$ is the series inductance per unit length and and $C_{T L}$ is the shunt capacitance per unit length of a transmission line. Angular frequency $\omega$ equals $2 \pi f$. The reflection coefficient $\Gamma$ of a transmission line is 


$$
\Gamma=\frac{Z_{t d r}-Z_{i n}}{Z_{t d r}+Z_{i n}}
$$

where $Z_{t d r}$ is the impedance of the piezoelectric resonator. Variable $Z_{i n}$ is the impedance reflected from the coax, which is defined [16] as

$$
Z_{\text {in }}=Z_{0} \frac{Z_{t d r}+j Z_{0} \tan (\beta l)}{Z_{0}+j Z_{t d r} \tan (\beta l)}
$$

where characteristic impedance $Z_{0}$ of a transmission line is equal to $\sqrt{\frac{L_{T L}}{C_{T L}}}$. Substituting $z=-l$ into equation (3.7), we have [16]

$$
V(-l)=V^{+}\left(e^{j \beta l}+\Gamma e^{-j \beta l}\right) .
$$

Rearranging it, we have an expression of the incident voltage [16]

$$
V^{+}=\frac{V(-l)}{\left(e^{j \beta l}+\Gamma e^{-j \beta l}\right)} .
$$

At the input of the transmission line, $z=-l$, voltage $V$ is expressed as [16]

$$
V(-l)=\frac{V_{g} Z_{i n}}{Z_{g}+Z_{i n}}
$$

Substituting $z=0$ into equation (3.7), it becomes

$$
\begin{aligned}
V(0) & =V^{+}(1+\Gamma) \\
& =V(-l)\left[\frac{1}{\left(e^{j \beta l}+\Gamma e^{-j \beta l}\right)}\right][1+\Gamma]
\end{aligned}
$$

Substituting $V(-l)$ into the previous equation, we have an expression for the voltage $V(0)$ at the piezoelectric resonator.

$$
V_{t d r}=V(0)=\left[\frac{V_{g} Z_{i n}}{Z_{g}+Z_{i n}}\right]\left[\frac{1}{\left(e^{j \beta l}+\Gamma e^{-j \beta l}\right)}\right][1+\Gamma]
$$

The attenuation between transmitted and received signals in $\mathrm{dBV}$ is given by

$$
\text { Attenuation }(d B V)=20 \log _{10}\left(\frac{V_{a d j}}{V_{t d r}}\right) \text {, }
$$

where voltage $V_{a d j}$ denotes the received signal, which was adjusted by the sensitivity of the hydrophone. We also have 


$$
V_{a d j}=V_{h} \frac{M(f=1 M H z)}{M(f)} .
$$

where $M(f)$ is the sensitivity of the hydrophone corresponding to a particular frequency, and $V_{h}$ represents the measured results.

\subsection{Results}

The transducer was excited with $1 \mathrm{MHz}, 3 \mathrm{MHz}$, and $5 \mathrm{MHz}$ sinusoidal signals in two different levels, $2 \mathrm{~V}$ and $20 \mathrm{~V}$. The results for $2 \mathrm{~V}$ case were summarized in Table 3.1. The difference of attenuation between $1 \mathrm{MHz}$ and $3 \mathrm{MHz}$ was $9.6 \mathrm{dBV}$, and the difference between $1 \mathrm{MHz}$ and $5 \mathrm{MHz}$ was $14.9 \mathrm{dBV}$. In other words, when a $3 \mathrm{MHz}$ sinusoidal signal is applied to a piezoelectric plate, compared with a $1 \mathrm{MHz}$ sinusoidal signal with the same electrical level, the $3 \mathrm{MHz}$ signal will be mechanically attenuated further by $9.6 \mathrm{dBV}$ in the acoustic pressure field. Similarly, a $5 \mathrm{MHz}$ signal will be mechanically attenuated further by $14.9 \mathrm{dBV}$ in the acoustic pressure field. The result of the $20 \mathrm{~V}$ case can be found in the Appendix B. Errors are mainly due to manually locating the focal spot, and reading errors.

Table 3.1: Attenuation of harmonics with $2 V_{p-p}$ sinusoidal signals.

\begin{tabular}{ccccc}
\hline $\mathrm{f}(\mathrm{MHz})$ & $V_{g}$ & $V_{\text {in }}$ & $V_{h}$ & Attenuation \\
\hline 1 & $2.075 \mathrm{~V}$ & $1.088 \mathrm{~V}$ & $21.94 \mathrm{mV}$ & $-33.9 \mathrm{dBV}$ \\
3 & $2.020 \mathrm{~V}$ & $0.503 \mathrm{~V}$ & $8.007 \mathrm{mV}$ & $-43.5 \mathrm{dBV}$ \\
5 & $2.020 \mathrm{~V}$ & $0.2145 \mathrm{~V}$ & $7.65 \mathrm{mV}$ & $-48.8 \mathrm{dBV}$ \\
\hline
\end{tabular}




\section{Chapter 4}

\section{Amplifier Design}

\subsection{Introduction}

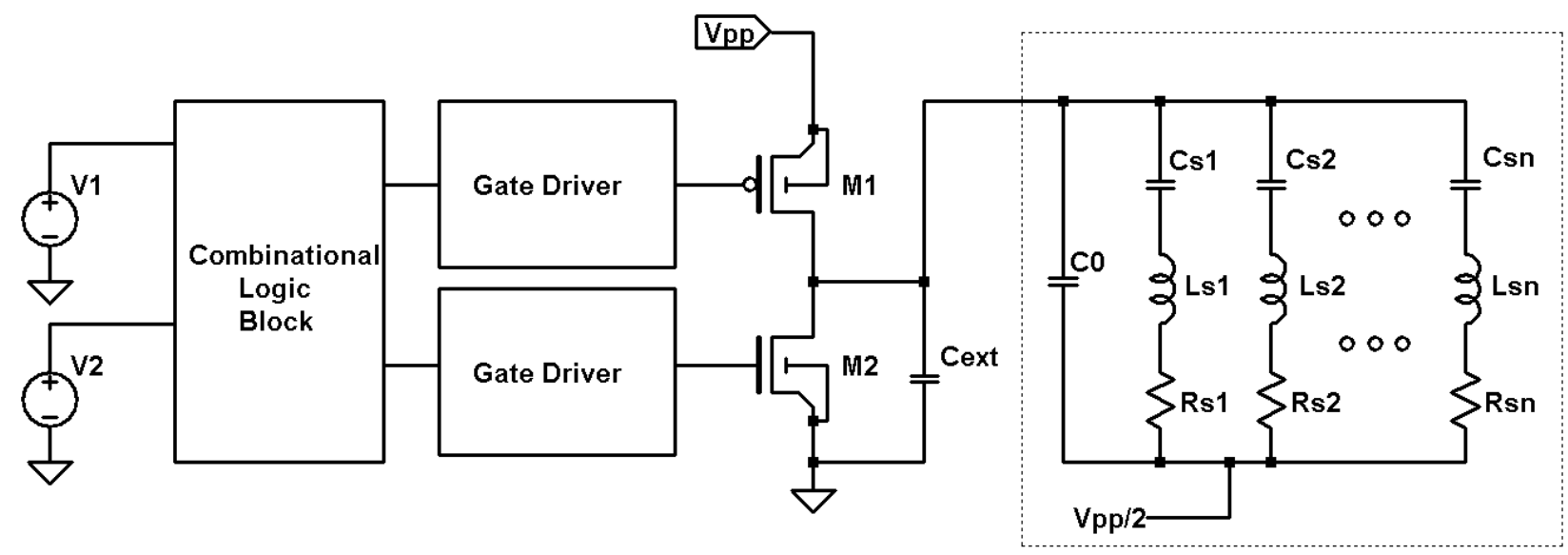

Amplifier

Transducer

Figure 4.1: Block diagram of the integrated amplifier for ultrasound transducer.

This chapter covers details of the circuit design, analysis of the class DE amplifier, methods of CMOS parameter extraction, and the design of transistors and gate drivers. The completed block diagram of the final design is illustrated in Figure 4.1. We combine the output stage, level shifters, and combinational logic blocks to synthesize the integrated amplifier. The output stage comprises two power transistors and is directly connected to an external capacitor $C_{\text {ext }}$ and an 
ultrasound transducer. The extra capacitance provided by $C_{e x t}$ stabilizes the behaviour of the transducer observed from the amplifier when the transducer is subjected to thermal expansion [13]. The gate driver comprises a level shifter and an output stage to produce a $20 \mathrm{~V}$ swing. A combinational logic circuit block is located prior to the gate drivers and is responsible for power stage on-off functioning and faulty signal filtering. Sources $v_{1}$ and $v_{2}$ are signal generators. This

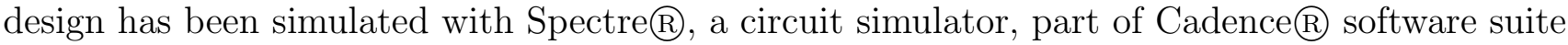
for professional custom IC design and validation.

A class DE amplifier was used for the integrated amplifier because it can be used in high frequency applications [17]. It is efficient and (in this case) does not require inductors. The performance of a class D amplifier at high frequencies can be improved with ZVS and ZDS. By reducing the turn-on ratio $\mathrm{D}$ of each transistor from 0.5 to 0.25 , it allows the network to precisely discharge the shunt capacitor before the next transistor conducts. This idea has been established for class DE amplifiers and will be described in Section 4.2.

\subsubsection{CMOS process kit}

The design is implemented with the $0.8 \mu \mathrm{m}$ CMOS process $5 / 20 \mathrm{~V}$ kit offered by TeledyneDALSA Semiconductor. It allows both standard $5 \mathrm{~V}$ and $20 \mathrm{~V}$ transistors to be fabricated in the same die. Complementary middle voltage transistors permit users to create a compact design, integrating a high power output driver and taking advantage of complementary circuit topologies with a wider voltage range [18]. Figures 4.2 (a) and (b) show the symbols of a standard $5 \mathrm{~V}$ PMOS and NMOS. Figures 4.2 (c) and (d) illustrate the symbols of $20 \mathrm{~V}$ middle MOSFETs.

\subsection{Class DE Amplifier}

A class DE amplifier with one shunt capacitor has been chosen for the output stage of the HIFU IC. Its circuit topology is shown in Figure 4.3 [5]. In order to simplify the analysis of a class DE amplifier, several assumptions have been made:

- All components, such as capacitor, inductor and resistor are ideal [17].

- Transistors are replaced by ideal switches . They have no parasitic capacitance, no channel resistance, no turn-on delays, no turn-off delays and infinity resistance while turned off [5]. 


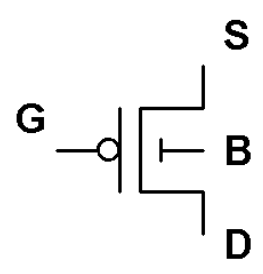

(a)

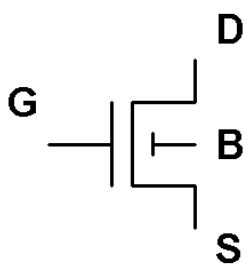

(b)

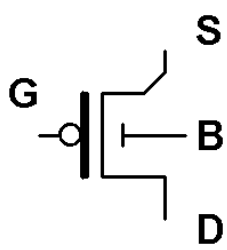

(c)

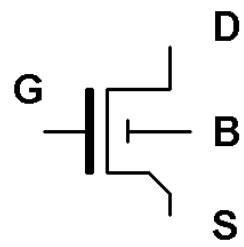

(d)

Figure 4.2: Symbol of high-voltage and low voltage MOSFETs. (a) LV PMOS (b) LV NMOS (c) HV PMOS (d) HV NMOS

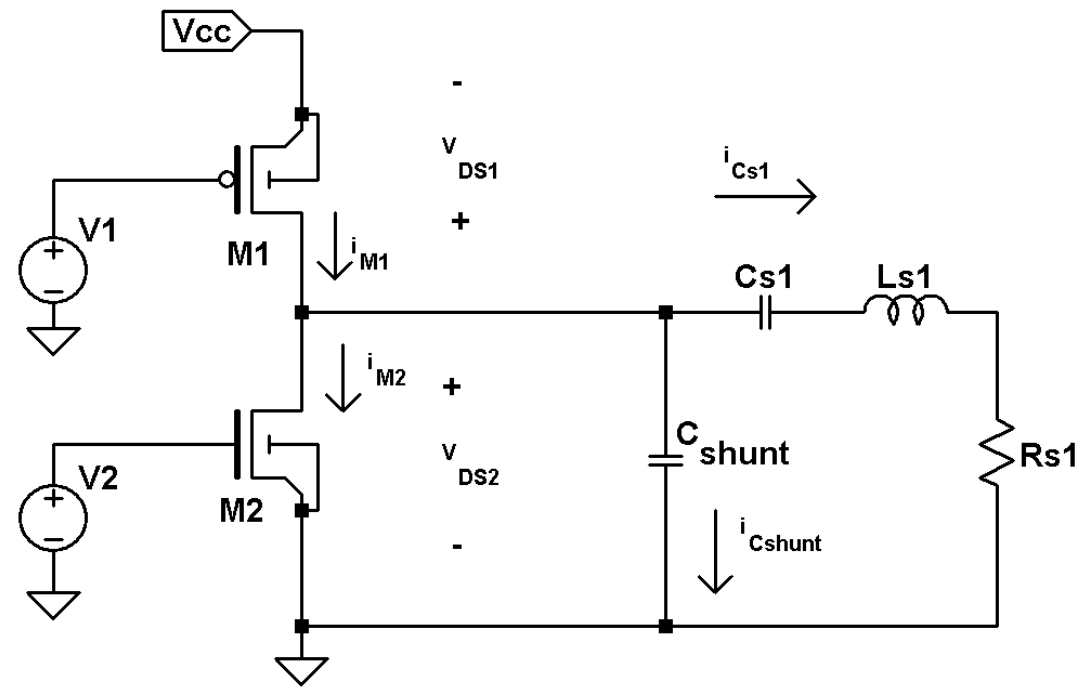

Figure 4.3: Topology of class DE amplifier with one shunt capacitor [5]. 


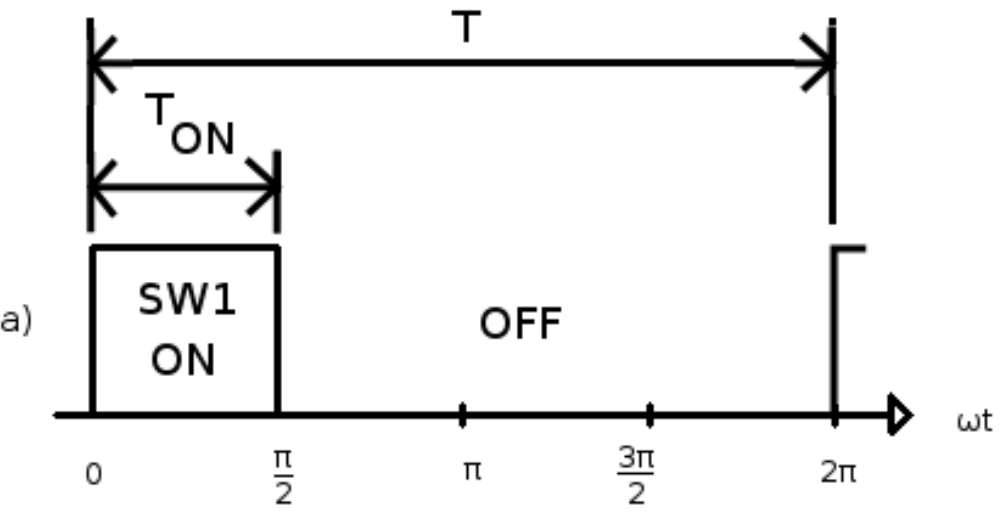

(b)

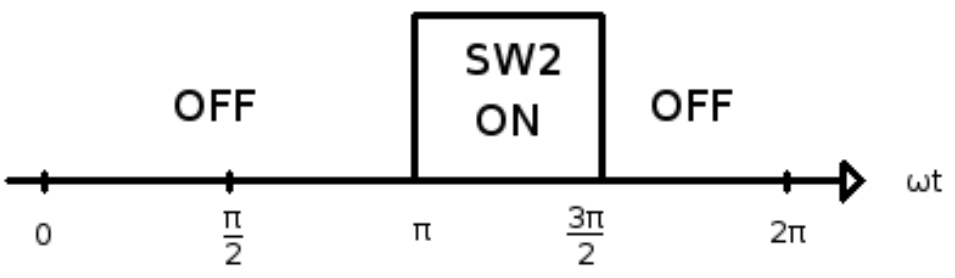

(c)
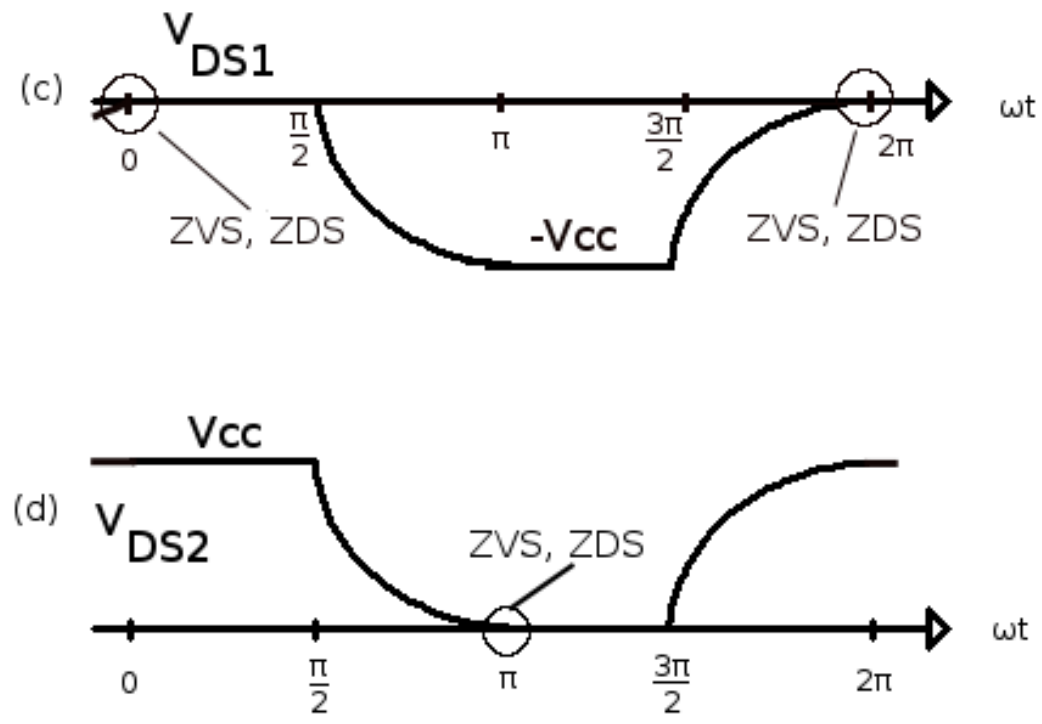

Figure 4.4: Voltage waveforms of class DE amplifier [5]. (a) and (b) The switch-on timing diagram of switches M1 and M2. (c) and (d) The voltage waveforms of switches M1 and M2. They are represented by symbols $v_{D S 1}$ and $v_{D S 2}$ respectively. 
(a)

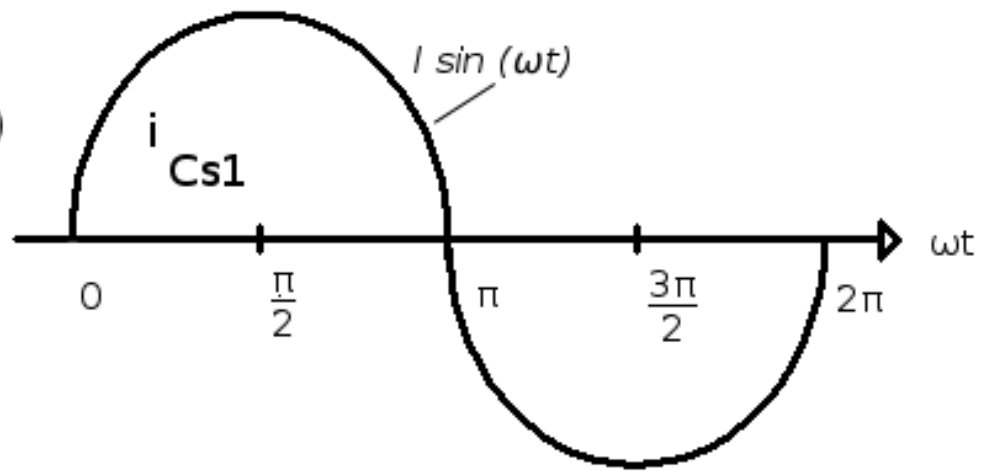

(b)

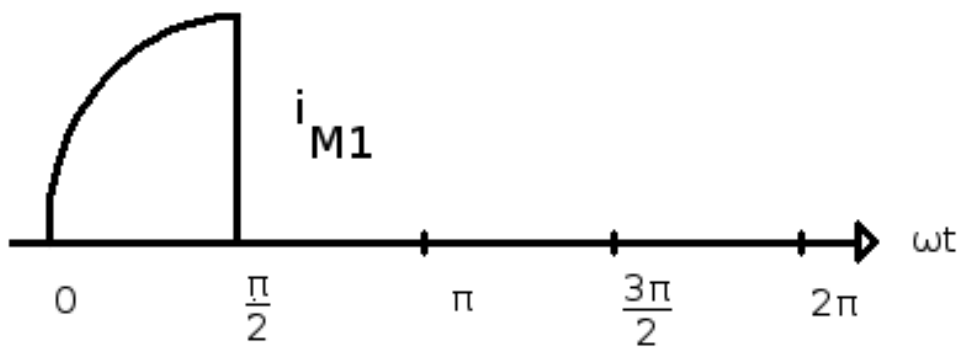

(c)

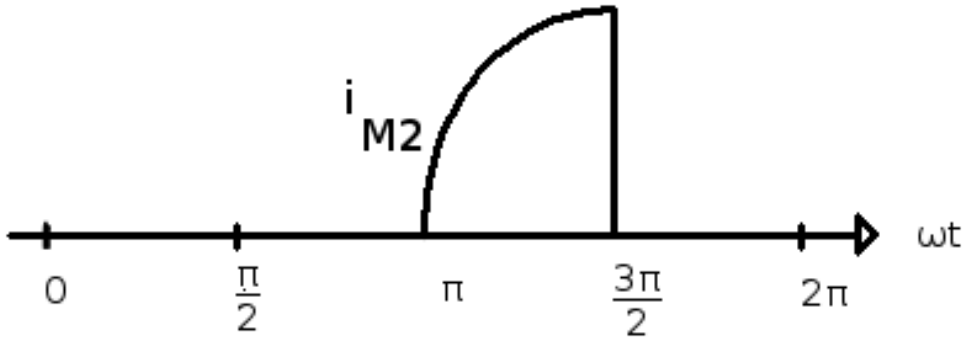

(d)

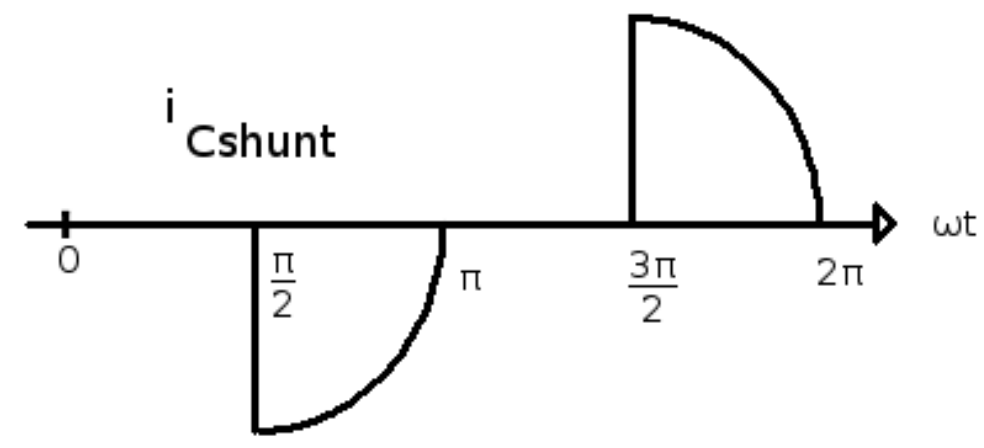

Figure 4.5: Current waveforms of class DE amplifier [5]. (a) The output current $i_{C_{s 1}}$. (b) and (c) Current waveforms across terminals of switches M1 $\left(i_{M 1}\right)$ and M2 $\left(i_{M 2}\right)$. (d) Current waveforms of shunt capacitor $i_{C_{\text {shunt }}}$. 
- The quality factor $Q=\frac{\omega L_{s 1}}{R_{s 1}}$ of the load network is more than $2.5[5]$, high enough to produce a sinusoidal output current $i_{C_{s 1}}$.

- The switch-on duty ratio (D) is exactly one quarter of a period of the operating frequency [5], [19], [20]. Variable D is the ratio of switch's conducting time to its period of working frequency: $D=\frac{T o n}{T}$. Period $\mathrm{T}$ and switch-on duration $T_{O N}$ are illustrated in Figure 4.4 (a). Each switch is manipulated by an ideal source.

Based on these assumptions, voltage waveforms, current waveforms and equivalent circuits are constructed and illustrated in Figure 4.4, Figure 4.5 and Figure 4.6 [5]. At any time, the output current $i_{C_{s 1}}$ can be expressed as

$$
i_{C_{s 1}}(\omega t)=I \sin (\omega t+\phi)
$$

where $I=\frac{V c c}{R_{S 1}}$ is the amplitude of current that flows through the series resonant network; $\phi$ is phase shift; $\omega$ is the angular frequency and variable $t$ denotes time. Since the $i_{C_{s 1}}$ curve goes through zero at $\omega t=\pi$, the phase angle $\phi$ is 0 . Thus, the expression for output current becomes

$$
i_{C_{s 1}}=I \sin (\omega t)
$$

The operation of a class DE amplifier in steady state can be divided into four intervals. Figure 4.6 illustrates the corresponding equivalent circuit in each period.

Interval 1, between 0 radians and $\frac{\pi}{2}$. Switch M1 conducts; Switch M2 is turned off. The equivalent circuit is shown in Figure 4.6(a). Current $i_{M 1}$ (Figure 4.5(a) ) slowly rises from zero at 0 radians and reaches its peak at $\frac{\pi}{2}$. Terminals of switch M2 are at the same potential $(V c c)$ during this period; therefore, no current will flow through capacitor $C_{\text {shunt }}$. Current $i_{M 1}$ becomes the only source that feeds the series resonant network. Before 0 radians, voltage across switch M1 $v_{D S_{1}}(\omega t)$ is increasing. It eventually reaches the ground potential at 0 radians. At the same instance, switch M1 is turned on. Since both of its terminals are at the ground potential, the switching losses is zero (ZVS). The derivative of $v_{D S_{1}}(\omega t)$ is also zero at this point (ZVD).

The advantage of achieving ZVC and ZDS is to eliminate switching losses while the switch is turned on. 


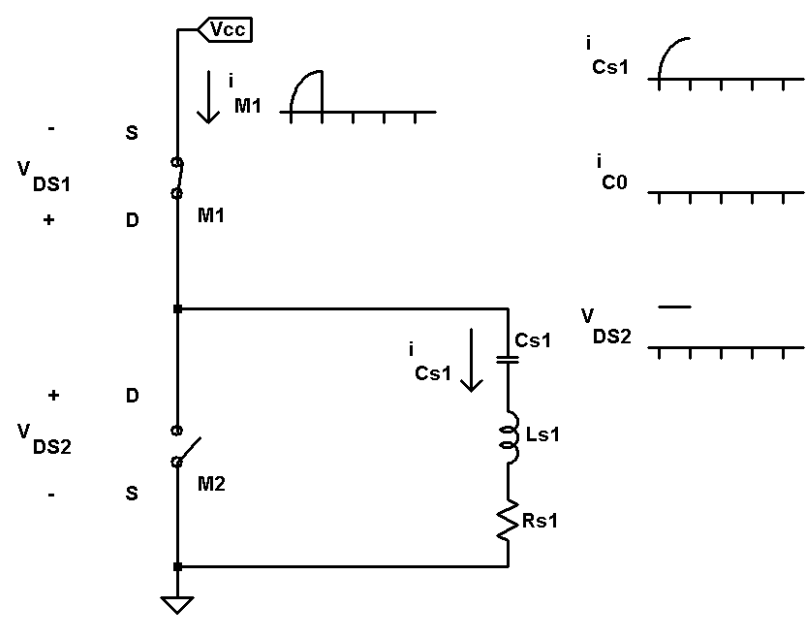

(a)

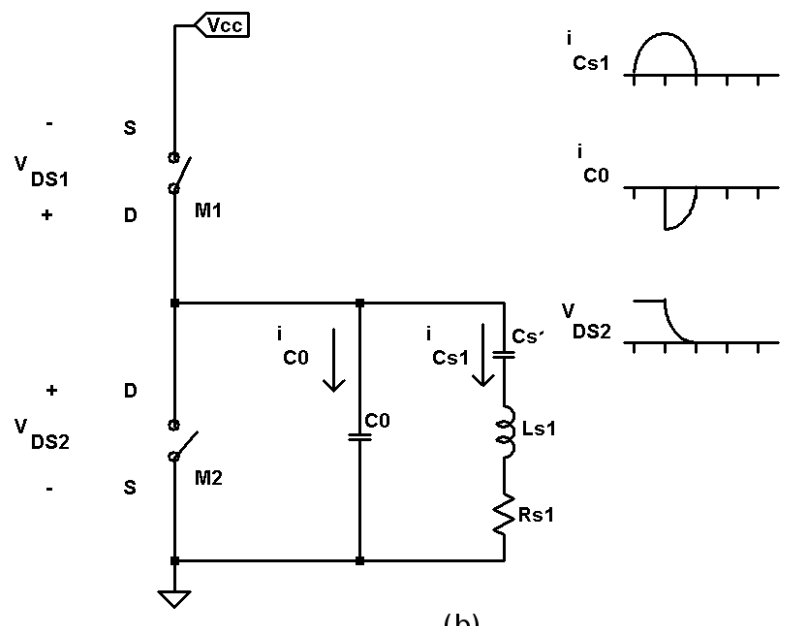

(b)

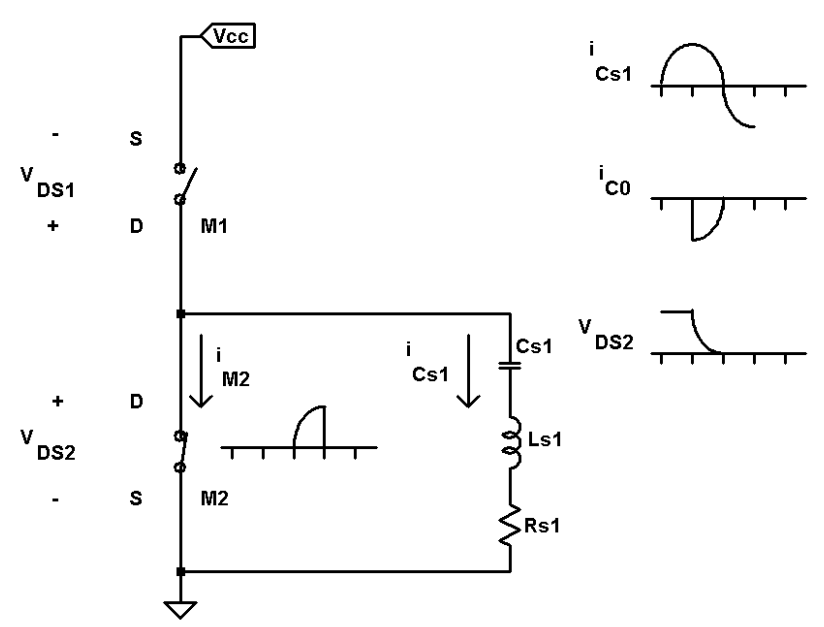

(c)

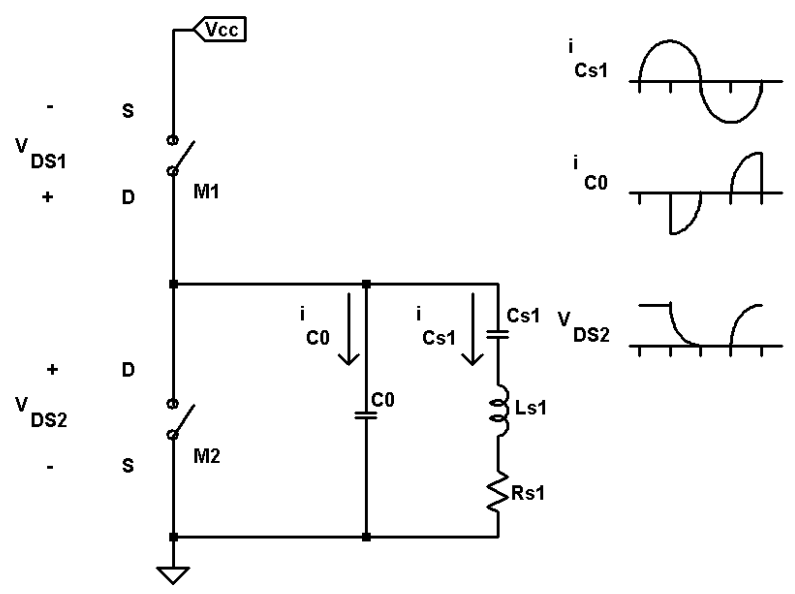

(d)

Figure 4.6: Equivalent circuit of class DE amplifier at each stage and waveforms [5]. (a) Between 0 radians and $\frac{\pi}{2}$, switch M1 turns on. (b) Between $\frac{\pi}{2}$ and $\pi$, both switches are turned off. (c) Between $\pi$ and $\frac{3 \pi}{2}$, switch M2 conducts. (d) Between $\frac{3 \pi}{2}$ and $2 \pi$, both switches are opened. 
Interval 2, between $\frac{\pi}{2}$ and $\pi$. Switch M1 and switch M2 are turned off. No current will flow through any switches during this period. The equivalent circuit is shown in Figure 4.6(b). At $\frac{\pi}{2}$, current $i_{M 1}$ drops to zero immediately. Shunt capacitor $C_{\text {shunt }}$ continues to provide the output current instead, because the series inductor $L_{s 1}$ and the series capacitor $C_{s 1}$ resonate to maintain the continuity of the output current. The magnitude of output current $i_{C_{s 1}}$ falls form its peak at $\frac{\pi}{2}$ and goes through zero at $\pi$. Voltage across switch M2 $v_{D S_{2}}$ declines slowly from Vcc to zero and $v_{D S_{1}}$ decreases from zero to $-V c c$. The expressions for $i_{C_{s h u n t}}, i_{C_{s 1}}, i_{C_{0}}, v_{D S_{1}}$, and $v_{D S_{2}}$ are derived as follows [5]:

$$
i_{C_{\text {shunt }}}=\omega C_{\text {shunt }} \frac{d\left(v_{D S_{2}}\right)}{d(\omega t)}
$$

Rearranging equation (4.3),

$$
d\left(v_{D S_{2}}\right)=\frac{i_{C_{\text {shunt }}}}{\omega C_{\text {shunt }}} d(\omega t)
$$

Integrating both sides,

$$
v_{D S_{2}}(\omega t)=\frac{1}{\omega C_{\text {shunt }}} \int_{\frac{\pi}{2}}^{\omega t} i_{C \text { shunt }} d(w t)
$$

The current $i_{C_{s 1}}$ and the current of shunt capacitor $i_{C_{\text {shunt }}}$ are heading in opposite directions. Therefore, we have

$$
i_{C_{s 1}}(\omega t)=-i_{C_{\text {shunt }}}(\omega t)
$$

Substituting Equation (4.1) into the previous expression, we have

$$
i_{C_{\text {shunt }}}(\omega t)=-I \sin (\omega t)
$$

Substituting Equation (4.5) into Equation (4.7), gives

$$
v_{D S_{2}}(\omega t)=\frac{-I}{\omega C_{\text {shunt }}} \int_{\frac{\pi}{2}}^{\omega t} \sin (\omega t) d(w t)+V c c
$$

where the first term $\frac{I}{\omega C_{\text {shunt }}}=V c c$ because $V=I Z$ and $Z=\frac{1}{\omega C_{\text {shunt }}}$; the second term $V c c$ is the initial condition $v_{D S 2}\left(\frac{\pi}{2}\right)=V c c$. Solving Equation (4.8),

$$
\left.v_{D S_{2}}(\omega t)\right|_{\omega t=\frac{\pi}{2}} \text { to } \pi=V c c(\cos (\omega t)+1) .
$$


The voltage across switch $\mathrm{M} 1, v_{D S_{1}}(\omega t)$ is

$$
v_{D S_{1}}(\omega t)=v_{D S_{2}}(\omega t)-V c c .
$$

Substituting Equation (4.9) into previous equation gives

$$
\left.v_{D S_{1}}(\omega t)\right|_{\omega t=\frac{\pi}{2}} \text { to } \pi=V c c(\cos (\omega t))
$$

Interval 3, between $\pi$ and $\frac{3 \pi}{2}$. Switch M1 remains opened. The equivalent circuit is shown in Figure 4.6(c). Voltage $v_{D S_{1}}(\omega t)$ is maintained at -Vcc. At $\pi$, Switch M2 conducts with no potential difference between its terminals; this satisfies the conditions of ZVS and ZDS. The output current $i_{C_{0}}$ goes through zero and keeps decreasing. At $\frac{3 \pi}{2}, i_{c_{0}}$ reaches $-I$. Since M2 is conducting, no current flows through the shunt capacitor. Table 4.1 summarize the conditions of ZVS and ZDS for M1 and M2 [5].

Table 4.1: Conditions of ZVS and ZDS.

\begin{tabular}{lll} 
& Switch M1 & Switch M2 \\
\hline ZVS & $v_{D S 1}(2 \pi)=0$ & $v_{D S 2}(\pi)=0$ \\
ZDS & $\frac{d\left(v_{D S 1}(2 \pi)\right)}{d(\omega t)}=0$ & $\frac{d\left(v_{D S 2}(\pi)\right)}{d(\omega t)}=0$ \\
\hline
\end{tabular}

Interval 4, between $\frac{3 \pi}{2}$ and $2 \pi$. Both switches are closed. The equivalent circuit is illustrated in Figure $4.6(\mathrm{~d})$. At $\frac{3 \pi}{2}$, the shunt capacitor $C_{0}$ sources the output current, because $C_{s 1}$ and $L_{s 1}$ are resonating. Current $i_{C s 1}$ declines to zero at $2 \pi$. During this period, voltage $v_{D S_{1}}(\omega t)$ increases slowly from $-V c c$ to zero and voltage $v_{D S_{2}}(\omega t)$ increases from zero to $V c c$. Following a similar derivation as for Interval 2, expressions for $v_{D S_{1}}(\omega t)$ and $v_{D S_{2}}(\omega t)$ are:

$$
\left.v_{D S_{2}}(\omega t)\right|_{\omega t=\frac{2 \pi}{2}} \text { to } 2 \pi=V c c(\cos (\omega t))
$$

and

$$
\left.v_{D S_{1}}(\omega t)\right|_{\omega t=\frac{2 \pi}{2}} \text { to } 2 \pi=V c c(\cos (\omega t)-1) .
$$


Table 4.2: The expressions of class DE amplifier output voltage and current waveforms.

Symbols 0 to $\frac{\pi}{2} \quad \frac{\pi}{2}$ to $\pi \quad \pi$ to $\frac{3 \pi}{2} \quad \frac{3 \pi}{2}$ to $2 \pi$

Voltage across

$v_{D S_{1}}$

0

$V c c \cos (\omega t)$

- Vcc

$V c c(\cos (\omega t)-1)$

M1

Voltage across

$v_{D S_{2}}$

Vcc

$V c c(\cos (\omega t)+1)$

0

$V c c(\cos (\omega t)-1)$

M2

Output current

$i_{C_{s 1}} \quad I \sin (\omega t)$

$I \sin (\omega t)$

$I \sin (\omega t)$

$I \sin (\omega t)$

Current flows

$i_{D 1} \quad I \sin (\omega t)$

0

0

0

through M1.

Current flows

$i_{D 2}$

0

0

$I \sin (\omega t)$

0

through M2.

Current flows through

$i_{c_{0}}$

0

$-I \sin (\omega t)$

0

$-I \sin (\omega t)$

shunt capacitor. 
It is important to point out that this analysis only considers an ideal scenario that excludes real parameters such as parasitic capacitance, channel resistance, and switching delays. In the circuit design, drain capacitance should be estimated because it directly affects the switching conditions. It will add up to the shunt capacitance to form a total capacitance in calculations. Moreover, capacitance is considered to be a constant while voltage is varying. Other factors such as channel resistance, and turn-on and turn-off delays are inevitable in reality. The measured efficiency of class DE amplifiers in references $[17,19]$ is above $90 \%$ operating at $1 \mathrm{MHz}$ with an output power between $1 \mathbf{W}$ and $7 \mathbf{W}$. Losses are mainly due to switching losses and parasitic resistance [20]. Table 4.2 summarizes all expressions valid in each interval.

\subsubsection{Tuning of a practical class DE amplifier}

Since the class DE amplifier attaches to an ultrasound transducer instead of a designated load network, it will not function in ideal mode. In such a case, tuning will help designers to discover the best configuration, a combination of $\mathrm{D}$ and $C_{\text {shunt }}$, without jeopardizing too many

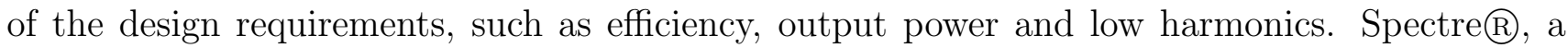
circuit simulator in the Cadence $\mathbb{R}$ suite, carried this out with the parametric analysis.

Ablulet [19] recommended a tuning procedure for the class DE amplifier. It involves varying circuit parameters including switch-on duty ratio $\mathrm{D}$, inductance $L_{s 1}$, and capacitance $C_{s 1}$. The author claimed that a practical class DE amplifier will not operate in optimum condition once built. Instead, by varying $\mathrm{D}$ and phase angle $\psi$ of the series resonant network, the best possible working condition can be obtained through analysis. The equation of phase angle $\psi=\arctan \left(\frac{1}{R_{s 1}}\left(2 \pi f L_{s 1}-\frac{1}{2 \pi f C_{s 1}}\right)\right)$ implied that $\phi$ can be adjusted by series capacitance $C_{s 1}$ and inductance $L_{s 1}[19]$ in Figure 4.3. The range of D can be anywhere from 0.25 to 0.5. Figure 4.7 (a) summarizes Ablulet's ideas. Figure 4.7(b) is what happen if the switching condition is optimum.

In Figure 4.7(a), the half U-shape is the valley of a sinusoidal waveform where its amplitude reached minimum. For optimum working conditions, the valley must be placed exactly at the 


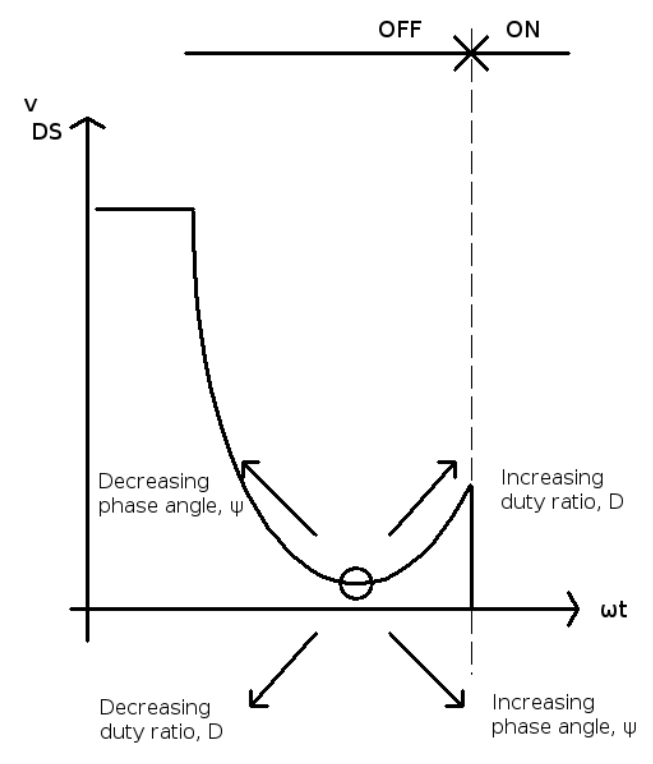

(a)

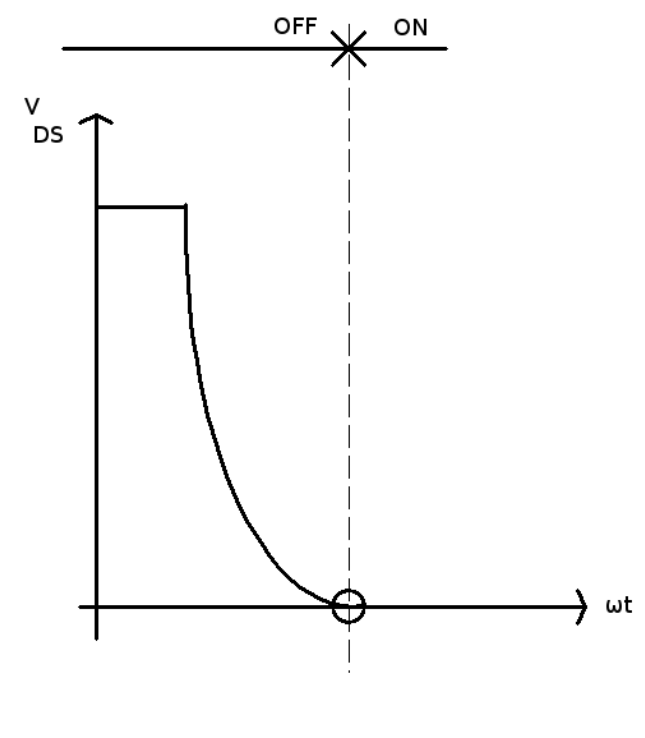

(b)

Figure 4.7: Tuning procedure for class DE amplifier [19]. (a) Tuning procedure (b) Optimum condition. intersection of the boundary of on-off region (the vertical dotted line in Figure 4.7) and the horizontal axis $\omega t$. To move the circle along the left diagonal, the phase angle should be varied. The phase angle is equal to the arctan of the residual reactance of capacitance $C_{s}$ and inductance $L_{s}$ divided by resistance $R_{s}$. On the other hand, for the trough travelling along the right diagonal, D should be varied. Decreasing D moves the trough to the lower left hand corner, and vice versa.

The author concluded that, unlike a class E amplifier, it is nearly impossible for a class DE amplifier to achieve very precise switching operations with real components. The practical range of $\mathrm{D}$ is between 0.25 and 0.33 [19]. In addition, the optimum point of class DE power amplifier does not occur at maximum output or input [19]. The above analysis is limited to a constant output capacitance scenario. 


\subsubsection{Calculations}

\section{External Capacitance for class DE amplifier}

As shown in Figure 4.1, the output network of the class DE amplifier is composed of an ultrasound transducer connecting in parallel with an external capacitor $\left(C_{\text {ext }}\right)$. The magnitude of shunt capacitance should be determined because it determines the reasonable external capacitance that should be used in tuning. The expression of the shunt capacitance is derived as follows [5]:

$$
\begin{aligned}
I_{D C} & =\frac{1}{2 \pi} \int_{0}^{\frac{\pi}{2}} i_{R_{s 1}} d(\omega t) \\
& =\frac{1}{2 \pi} \int_{0}^{\frac{\pi}{2}} I_{m} \sin (\omega t) d(\omega t) \\
& =\frac{I_{m}}{2 \pi} \\
& =\frac{V c c \omega C_{\text {shunt }}}{2 \pi}
\end{aligned}
$$

where $I_{D C}$ stands for DC input current and $I_{m}$ denotes the peak magnitude of sinusoidal current. The peak voltage across $R_{s 1}$ is derived using Fourier analysis [5]:

$$
\begin{aligned}
V_{m}= & \frac{1}{\pi} \int_{0}^{2 \pi} v_{D S 2} \sin (\omega t) d(\omega t) \\
= & \frac{1}{\pi}\left[\int_{0}^{\frac{\pi}{2}} V c c \sin (\omega t) d(w t)+\int_{\frac{\pi}{2}}^{\pi} V c c(\cos (\omega t)+1) \sin (\omega t) d(w t)+\cdots\right. \\
& \left.\quad+\int_{\frac{3 \pi}{2}}^{2 \pi} V c c \cos (\omega t) \sin (\omega t) d(w t)\right] \\
= & \frac{1}{\pi}\left[V c c+\frac{V c c}{2}-\frac{V c c}{2}\right] \\
= & \frac{V c c}{\pi}
\end{aligned}
$$

Assuming 100\% efficiency, the power of DC supply is completely transferred to the load resistor $[5]:$

$$
P=\frac{V_{m}{ }^{2}}{2 R_{s 1}}=\left(\frac{V c c}{\pi}\right)^{2} \frac{1}{2 R_{s 1}}=V c c I_{D C}=V c c\left(\frac{V c c \omega C_{\text {shunt }}}{2 \pi}\right),
$$

where P stands for power. Simplify the above expression; we have,

$$
C_{\text {shunt }}=\frac{1}{\pi \omega R_{s 1}}
$$


where $\omega=2 \pi f_{s}$ is the series resonant frequency of the fundamental branch; $R_{s 1}$ is the resistor of the fundamental branch [5]. In this case, the external capacitor $C_{\text {ext }}$ is given by:

$$
\begin{gathered}
C_{\text {ext }}=C_{\text {shunt }}-C_{0}-C_{\text {drain }_{M 1}}-C_{\text {drain }_{M 2}} \\
C_{\text {ext }}=\frac{1}{\pi\left(2 \pi f_{s}\right) R_{s 1}}-C_{0}-C_{\text {drain }_{M 1}}-C_{\text {drain }_{M 2}},
\end{gathered}
$$

where variable $C_{0}$ is the transducer's static capacitor. Variables $C_{d r a i n_{M 1}}$ and $C_{d r a i n_{M 2}}$ denote the drain capacitance of the power MOSFETs M1 and M2.

\section{Drain Capacitance of a MOSFET}

The drain capacitance of a MOSFET can be estimated as follows.

$$
C_{\text {drain }}=\left(L_{\text {drain }} \cdot W\right) \cdot c j+2\left(W+L_{\text {drain }}\right) \cdot c j s w
$$

where variable $W$ denotes the width of a MOSFET gate, and $L_{\text {drain }}$ is the length of drain; process parameters $c j$ and $c j s w$ denote junction capacitance and junction sidewall capacitance respectively. Since all process parameters use in simulation model are confidential, we can only

provide the results here. The estimated drain capacitance of M1 and M2 were $18.43 p F$ and $24.31 p F$ respectively. Substituting them into (4.17) gives

$$
\begin{aligned}
C_{\text {ext }} & =\frac{1}{\pi(2 \pi)(0.982 M H z)(31.88 \Omega)}-1.388 n F-18.43 p F-24.31 p F \\
& =273 p F
\end{aligned}
$$

\section{Gate Capacitance of a MOSFET}

The gate capacitance of a MOSFET can be estimated as follows.

$$
C_{\text {gate }}=W \cdot L \cdot \operatorname{cox}+W(\text { cgdo }+ \text { cgso })
$$

where $C_{\text {gate }}$ is the gate capacitance; process parameters cgdo denotes gate-drain parasitic capacitance and cgso represents gate-source parasitic capacitance. The variable Cox is defined as follows.

$$
\operatorname{Cox}=\frac{\varepsilon_{0} \varepsilon_{r}}{t o x}
$$


where $\varepsilon_{0}=\frac{10^{-9}}{36 \pi} F / m$ and $\varepsilon_{r}=3.97$ represent the permittivity of free space and the relative permittivity of silicon oxide; process parameter tox denotes the gate oxide thickness. The estimated gate capacitance of M1 and M2 were $28.7 p F$ and $44.1 p F$ respectively.

\section{$K^{\prime}$ and threshold voltage}

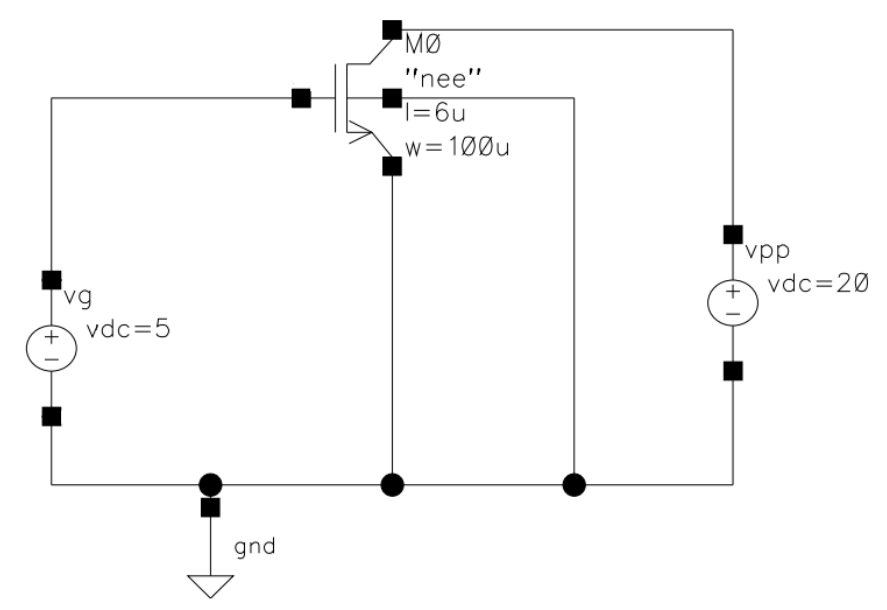

Figure 4.8: Schematic of technological parameters extraction.

Electrical properties such as K' parameter and threshold voltage of a MOSFET are gathered using curve fitting method. This can be done by simulating a test circuit in Spectreß and comparing a theoretical model with simulation results. Figure 4.8 illustrates a test circuit for eliciting threshold voltage and K' parameters from a MOSFET, respectively. The drain current of a MOSFET is defined as follows.

$$
I_{D_{m}}=\frac{K^{\prime}}{2} \frac{W}{L_{e f f}}\left(V_{G S}-V_{t}\right)^{2} \frac{1}{1+\theta\left(V_{G S}-V_{t}\right)}
$$

where $I_{D_{m}}$ is the measured drain current; W is the width of gate; $V_{G S}$ stands for the potential between gate and source terminals. Variable $V_{t}$ denotes threshold voltage of MOSFET; $L_{e f f}$ is the effective length of gate and it is defined as follows:

$$
L_{e f f}=L-2 \cdot \operatorname{lint}
$$




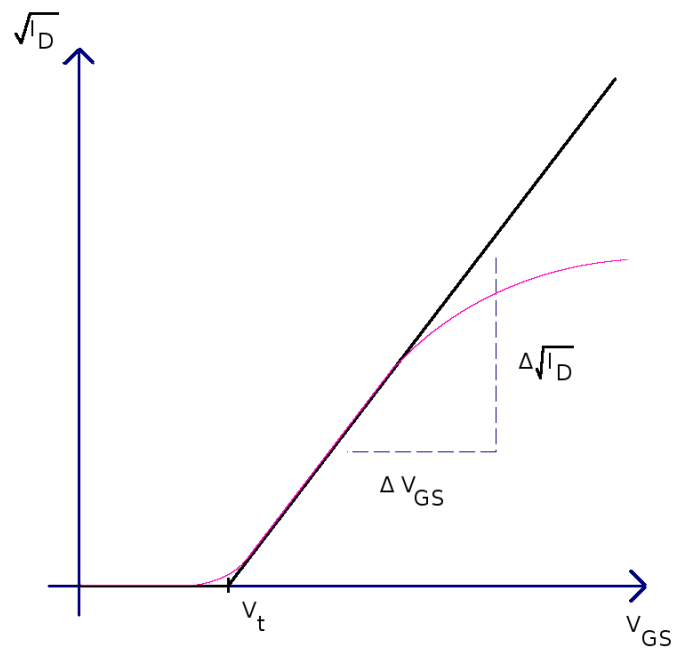

Figure 4.9: Square root of MOSFET drain current $\sqrt{I_{D}}$ as a function of gate-to-source voltage $V_{G S}$. Top (black): Drain current based on Equation (4.23). Bottom (Magenta): A more realistic $\sqrt{I_{D}}$ versus $V_{G S}$ curve rolls off as $V_{G S}$ increases due to mobility degradation.

where lint is the length offset parameter, a process parameters in BSIM model. The factor $\frac{1}{1+\theta\left(V_{G S}-V_{t}\right)}$ is introduced to compromise the linear behaviour near the end of the $\sqrt{I_{D}}$ versus $V_{G S}$ curve due to mobility degradation. It is indicated by the magenta colour line in Figure 4.9. Taking the square root on both sides of Equation (4.21), it becomes

$$
\sqrt{I_{D}}=\sqrt{\frac{K^{\prime}}{2} \frac{W}{L_{e f f}}}\left(V_{G S}-V_{t}\right) \sqrt{\frac{1}{1+\theta\left(V_{G S}-V_{t}\right)}}
$$

When $V_{G S}$ is slightly higher than $V_{t}$, the correction factor $\sqrt{\frac{1}{1+\theta\left(V_{G S}-V_{t}\right)}} \approx 1$. Without the correction factor, rewriting Equation (4.22), we have

$$
\sqrt{I_{D_{t}}}=\sqrt{\frac{K^{\prime}}{2} \frac{W}{L_{e f f}}}\left(V_{G S}-V_{t}\right) .
$$

where $I_{D_{t}}$ is the straight line that represents the theoretical drain current in Figure 4.9. The factor $\sqrt{\frac{K^{\prime}}{2} \frac{W}{L_{e f f}}}$ denotes the slope of the line. Such that,

$$
\frac{\Delta \sqrt{I_{D_{m}}}}{\Delta V_{G S}}=\text { slope }=\sqrt{\frac{K^{\prime}}{2} \frac{W}{L_{e f f}}}
$$

Solving for $K^{\prime}$, we have

$$
K^{\prime}=\frac{2 \cdot L(\text { slope })^{2}}{W}
$$


Dividing Equation (4.23) by Equation (4.22) gives,

$$
\frac{\sqrt{I_{D} t}}{\sqrt{I_{D} m}}=\sqrt{1+\theta\left(V_{G S}-V_{t}\right)}
$$

Rearranging the equation, we have an expression for $\theta$ which is true for $V_{G S}>>V_{t}$,

$$
\theta=\frac{I_{D_{t}}-I_{D_{m}}}{I_{D_{m}} \cdot V_{G S}-I_{D_{m}} \cdot V_{t}}=\left(\frac{I_{D_{t}}}{I_{D_{m}}}-1\right)\left(\frac{1}{V_{G S}-V_{t}}\right)
$$

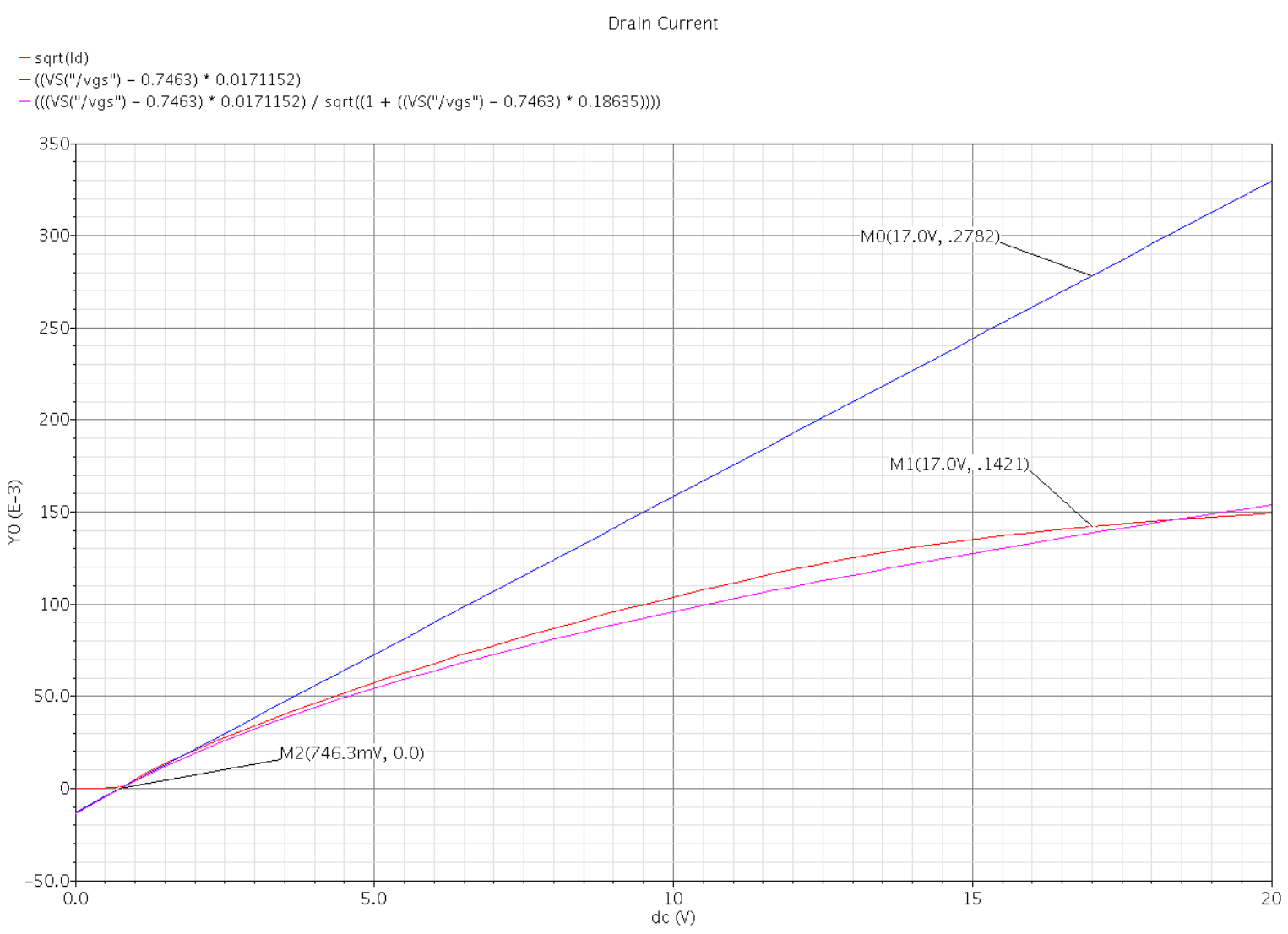

Figure 4.10: Simulation results of $\sqrt{I_{D}}$ as a function of $V_{G S}$ for HV NMOS, W $=100 \mu m, \mathrm{~L}=6 \mu \mathrm{m}$. Top (blue): Square root of theoretical drain current $\sqrt{I_{D_{t}}}$ based on Equation (4.23)

Middle (Red): Actual simulation results $\sqrt{I_{D_{m}}}$.

Bottom (Magenta): A recreated curve based on extracted parameters.

Figure 4.8 illustrates a HV NMOS with $\mathrm{W}=100 \mu \mathrm{m}$ and $L=6 \mu \mathrm{m}$. The simulation results and theoretical plots are shown in the Figure 4.10. Table 4.3 is a summary of extracted parameters, $K^{\prime}$, threshold voltage, and $\theta$, of the transistors that are used in this project. The 
last column of the table indicates an HV NMOS having a source-to-bulk voltage equal to $1 \mathrm{~V}$. Table 4.4 summarizes the gate capacitance and drain capacitance of transistors that were used in an earlier design.

Table 4.3: A summary of the extracted technological parameters.

\begin{tabular}{cc||ccccc}
\hline & units & LV PMOS & LV NMOS & HV PMOS & HV NMOS & HV NMOS \\
$L_{\text {eff }}$ & $\mu m$ & 0.8 & 0.8 & 4 & 6 & 6 \\
& & & & & & $\left(V_{S B}=1 \mathrm{~V}\right)$ \\
\hline$K^{\prime}$ & $\mu A / V^{2}$ & 32.01 & 95.71 & 16.60 & 33.98 & 40.20 \\
$V_{t}$ & $V$ & -0.6297 & 0.7044 & -0.967 & 0.7463 & 1.15 \\
$\theta$ & $V^{-1}$ & -0.2781 & 0.4328 & -0.1949 & 0.1864 & 0.238 \\
\hline
\end{tabular}

Table 4.4: Estimated size, gate capacitance, drain capacitance and channel resistance of transistors used in an earlier design.

\begin{tabular}{lcccccc}
\hline & M1 & M2 & M3 & M4 & M9 and M10 & M11 - M14 \\
\hline & HVPMOS & HVNMOS & HVPMOS & HVNMOS & HVNMOS & LV PMOS \\
Width & $10 m m$ & $10 ~ m m$ & $200 \mu m$ & $200 \mu m$ & $75 \mu m$ & $7 \mu m$ \\
Length & $4 \mu m$ & $6 \mu m$ & $4 \mu m$ & $6 \mu m$ & $6 \mu m$ & $0.8 \mu m$ \\
$C_{\text {gate }}$ & $28.7 p F$ & $44.12 p F$ & $0.5740 p F$ & $0.8825 p F$ & $0.3309 p F$ & $0.01765 p F$ \\
$C_{\text {drain }}$ & $18.43 p F$ & $24.31 p F$ & $0.3654 p F$ & $0.4853 p F$ & $0.1825 p F$ & $0.02222 p F$ \\
\hline
\end{tabular}




\section{Channel Resistance of MOSFET}

The channel resistance of both power transistors should be confirmed to be the same under identical biasing conditions. The expression of channel resistance can be derived from the expression of drain current of a MOSFET operated in triode mode.

$$
I_{D}=\frac{K^{\prime}}{2} \frac{W}{L_{e f f}} \frac{\left[2\left(V_{G S}-V_{t}\right) V_{D S}-V_{D S}{ }^{2}\right]}{1+\theta\left(V_{G S}-V_{t}\right)}
$$

While the channel is fully opened, $V_{D S}$ is much less than $2\left(V_{G S}-V_{t}\right)$. Therefore, the expression (4.28) becomes

$$
I_{D} \approx \frac{K^{\prime}}{2} \frac{W}{L_{e f f}} \frac{\left[2\left(V_{G S}-V_{t}\right) V_{D S}\right]}{1+\theta\left(V_{G S}-V_{t}\right)} .
$$

Rearranging it, we have the expression of the channel resistance.

$$
R_{c h}=\frac{V_{D S}}{I_{D}}=\frac{1+\theta\left(V_{G S}-V_{t}\right)}{\frac{K^{\prime}}{2} \frac{W}{L_{e f f}}\left(V_{G S}-V_{t}\right)} .
$$

The estimated channel resistance of M1 and M2 were respectively $4.17 \Omega$ and $4.07 \Omega$. The simulated channel resistance of both M1 and M2 were $3.41 \Omega$ and $3.39 \Omega$ correspondingly.

\subsection{Gate Driver}

The gate driver, converting $5 \mathrm{~V}$ digital signals to $20 \mathrm{~V}$ gate signals, comprises a level shifter and an output stage. Its schematic is illustrated in Figure 4.11 [21]. It requires two supply voltages of $5 \mathrm{~V}(V d d)$ and $20 \mathrm{~V}(V p p)$ respectively. The output stage consists of two high-voltage transistors M3 and M4, with the gate of the M3 routed to the level shifter. A basic level shifter offers high speed switching without the need for multiple driving signals. However, its major drawback is static power consumption which is approximately $2 \%$ of maximum output. The output (Vout) of the driver connects to the gate terminal of a power transistor; therefore, two gate drivers are needed. The functionality of each component explained below.

The purpose of using a level shifter is to raise the reference potential of $5 \mathrm{~V}$ input from ground to Vpp. It comprises four LV PMOS M11 - M13 as well as two HV NMOS M9 and M10. 


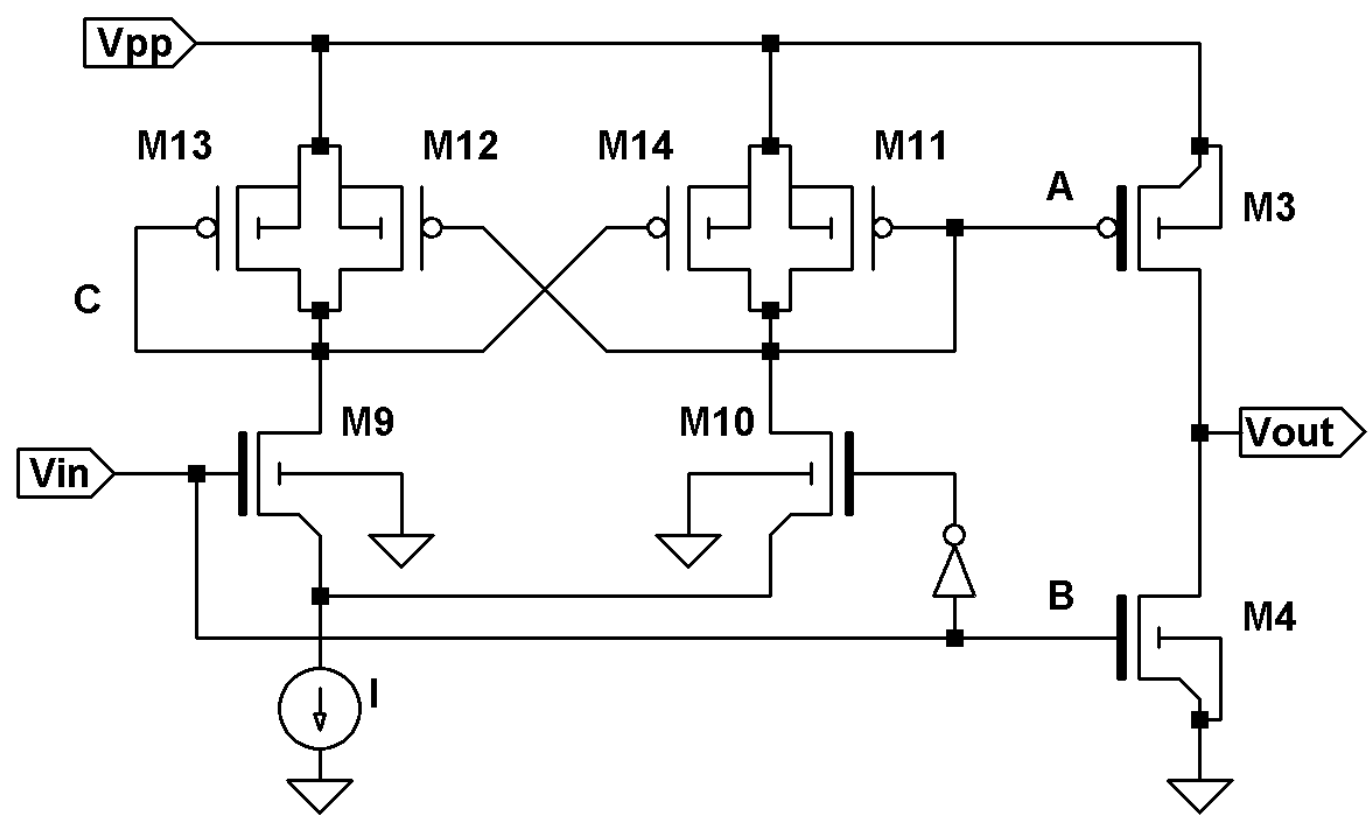

Figure 4.11: Schematic diagram of the gate driver for each power MOSFET [21].

Transistors M9, M12 and M13 as well as M10, M11, and M14 make two voltage mirrors. Their relationships in term of aspect ratio are listed as follows:

$$
\begin{aligned}
\left(\frac{W}{L}\right)_{11}= & \left(\frac{W}{L}\right)_{12}=\left(\frac{W}{L}\right)_{13}=\left(\frac{W}{L}\right)_{14} \\
& \left(\frac{W}{L}\right)_{9}=\left(\frac{W}{L}\right)_{10}
\end{aligned}
$$

Transistors M13 and M11 are connected as diodes, so they are always working in saturation mode.

When input Vin is logic high, transistors M13 and M9 conduct. The voltage $V_{C}=V p p-V d d$ turns on M14; then, voltage $V_{A}$ becomes $V p p$. Drain current of another voltage mirror is negligible because M14 and M10 are biased in cutoff mode. When input Vin is logic low, transistor M9 changes to cutoff mode. A negligible amount of current flows through the respective voltage mirror. Transistors M11 and M14 are biased in saturation mode. Voltage $V_{A}$ is now $V p p-V d d$. Voltage $V_{C}$ rises to Vpp. Current source (I) ensure that equal amount of current flows through the voltage mirrors and limits power consumption. 


\subsubsection{Calculations}

\section{Estimation of M3 and M4}

Transistors M3 and M4 control the rise time $t_{\text {rise }}$ and fall time $t_{\text {fall }}$ of the gate signals of the power transistors. Rise time is the duration that a voltage requires to increase from $10 \%$ to $90 \%$ of its maximum level. Fall time is defined as the duration that a voltage needs to decrease from $90 \%$ to $10 \%$ of its maximum level. Based on given $t_{\text {rise }}$ or $t_{\text {fall }}$, the size of M3 and M4 can be approximately estimated by differential equations [22] or a linear model $t=2.2 R_{c h} C_{\text {gate }}$. The details of using the differential equation method for estimating the width of M4 can be found in Appendix C. The estimated width of M3 and M4 were $215.3 \mu \mathrm{m}$ and $197.2 \mu \mathrm{m}$ correspondingly. After analysis of the simulation results, the width of $W_{M 3}$ and $W_{M 4}$ were aligned to $200 \mu m$ and $250 \mu m$ respectively.

\section{Voltage Mirror}

To determine the aspect ratio of M11 and M10, voltage $V_{A}$, slew rate and equivalent capacitance at node A are required. Transistors M11 and M10 are biased in saturation mode while conducting. Since $I_{D_{M 10}}=I_{D_{M 11}}$, their aspect ratio can be determined by the expression below [21]:

$$
\frac{K_{H V N}^{\prime}}{2} \frac{W_{10}}{L_{10}}\left(V_{G S}-V_{t_{H V N}}\right)^{2}=\frac{K_{L V P}^{\prime}}{2} \frac{W_{11}}{L_{11}}\left[\left(V p p-V_{A}\right)-V_{t_{L V P}}\right]^{2}
$$

where $K_{H V N}^{\prime}$ denotes the technological parameter for HV NMOS; $W_{n}$ and $L_{n}$ represent the width and length of the respective transistors. Voltage $V_{i n}$ denotes the peak-to-peak amplitude of the input signal, which equals $V d d ; V_{t_{L V}}$ is the threshold voltage of LV PMOS, and $V p p$ denotes the potential of the high voltage supply. However, Equation (4.30) cannot apply to our situation directly, because the mobility degradation correction is missing and a current mirror is attached at the end of the voltage mirror. That alters the threshold voltage of M9 and M10. Expression (4.30) was modified to fit our situation. We have,

$$
\frac{K_{H V N}^{\prime}}{2} \frac{W_{10}}{L_{10}} \frac{\left(V_{G S}-V_{t_{H V N} V_{S S}}\right)^{2}}{\left(1+\theta_{H V N}\left(V_{G S}-V_{t_{H V N} V B S}\right)\right)}=\frac{K_{L V P}^{\prime}}{2} \frac{W_{11}}{L_{11}} \frac{\left.\left(V p p-V_{A}\right)-V_{t_{L V P}}\right]^{2}}{\left(1+\theta_{L V P}\left(V_{G S}-V_{t_{L V P}}\right)\right)}
$$

where threshold voltage $V_{t_{H V N}{ }_{V B S}}$ is obtained through simulations. The subscripts $H V N$ and $L V P$ represent high-voltage and low-voltage respectively. At node A, the relationship of current, slew rate 
$S R$ and equivalent capacitance $C_{\text {load }_{A}}$ is defined as below:

$$
I_{D_{10}}=I_{D_{11}}=S R \cdot C_{\text {load }_{A}} .
$$

Capacitance $C_{\text {load }_{A}}$ at node A is the sum of $C_{\text {in }}$ of M3, M12 and M11 as well as $C_{\text {drain }}$ of M11, M14, and M10. In Figure 4.11, the equivalent capacitance at node $\mathrm{A}$ is

$$
\begin{aligned}
C_{A} & =C_{\text {gate }_{M 3}}+C_{\text {gate }_{M 11}}+C_{\text {gate }_{M 12}}+C_{\text {gate }_{M 10}}+C_{\text {drain }_{M 11}}+C_{\text {drain }_{M 14}} \cdot \\
& =0.6102 p F+0.01765 p F+0.01765 p F+0.3309 p F+0.01765 p F+0.01765 p F \\
& =1.01 p F
\end{aligned}
$$

The estimated equivalent capacitance at node B was $1.2 \mathrm{pF}$. The value of $C_{A}$ was rounded up to $1.25 \mathrm{pF}$ for the following estimation. For $1 \mathrm{MHz}, 25 \%$ duty ratio, $5 \mathrm{~V}$ peak-to-peak pulses with a rise time $\Delta t_{A}$ is set to $2 \%$ of the signal period, that gives us

$$
\begin{aligned}
\Delta t_{A} & =\frac{0.02 \cdot 0.25}{(1 \quad M H z)} . \\
\Delta t_{A} & =5 \mathrm{~ns}
\end{aligned}
$$

At node A, the slew rate $S R$ is obtained as follows:

$$
\begin{aligned}
S R & =\frac{V}{\Delta t_{A}} \\
S R & =\frac{5 V}{5 n s} \\
S R & =1000 \mathrm{~V} / \mu \mathrm{s}
\end{aligned}
$$

The drain current of M13 $i_{D_{M 13}}$ is calculated as below.

$$
\begin{aligned}
i_{D_{M 13}} & =-S R \cdot C_{A} \\
& =-1000 \mathrm{~V} / \mu \mathrm{s} \cdot 1.25 \mathrm{pF} \\
& =-1.25 \mathrm{~mA}
\end{aligned}
$$

Recall that the drain current of a MOSFET is defined by Equation (4.21).

$$
I_{D_{t}}=\frac{K^{\prime}}{2} \frac{W}{L}\left(V_{G S}-V_{t}\right)^{2} \frac{1}{1+\theta\left(V_{G S}-V_{t}\right)}
$$


Rearranging this, we have

$$
\frac{W}{L_{e f f}}=\frac{2 I_{D_{t}}}{K^{\prime}\left(V_{G S}-V_{t}\right)^{2}}\left(1+\theta \cdot\left(V_{G S}-V_{t}\right)\right) .
$$

The width of M13 is $7 \mu \mathrm{m}$ if the minimum channel length was chosen. The overdrive voltage of M13 is $-4.37 \mathrm{~V}$. Next, we can calculate M9 with Equation (4.31).

$$
\frac{K_{H V N}^{\prime}}{2} \frac{W_{9}}{L_{9}} \frac{\left(V_{G S}-V_{t_{H V N} V B S}\right)^{2}}{\left(1+\theta_{H V N}\left(V_{G S}-V_{t_{H V N} V B S}\right)\right)}=\frac{K_{L V P}^{\prime}}{2} \frac{W_{13}}{L_{13}} \frac{\left.\left(V p p-V_{A}\right)-V_{t_{L V P}}\right]^{2}}{\left(1+\theta_{L V P}\left(V_{G S}-V_{t_{L V P}}\right)\right)}
$$

Rearranging gives us

$$
W_{M 9}=\frac{W_{M 13}}{\frac{1+\theta_{M 13}\left(v_{A}-V p p-V_{t h_{M 13}}\right)}{1+\theta_{M 9(v b s)}\left(V_{G S}-V_{t h_{M 9(v b s)}}\right)} \frac{\frac{K_{M 9(v b s)}^{\prime}\left(v_{G S}-v_{t h_{M 9(v b s)}}\right)^{2}}{L_{e f f_{M 9}}}}{\frac{K_{M 13}^{\prime}}{L_{e f f_{M 13}}}\left(v_{A}-V p p-v_{t h_{M 13}}\right)^{2}}} .
$$

The estimated widths of M9 and M10 was $76.63 \mu \mathrm{m}$. The widths of M9 and M10 were adjusted to $70 \mu m$ in the final design.

\subsection{Current Mirror}

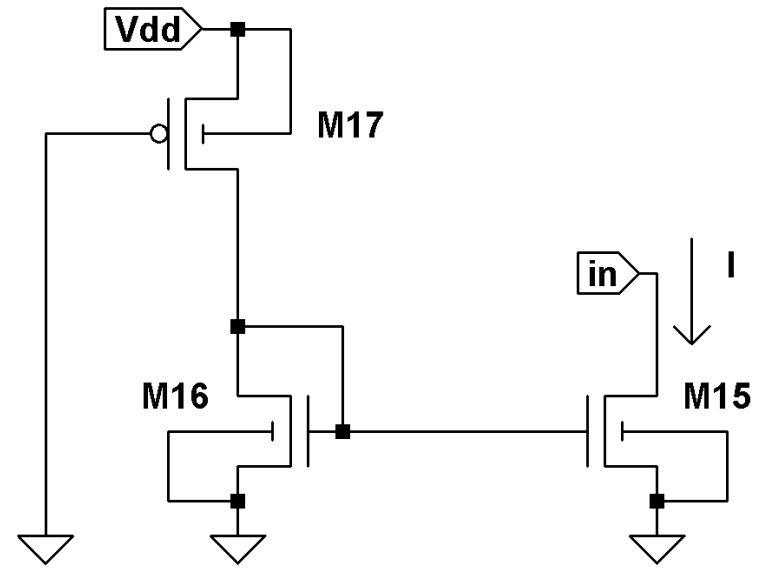

Figure 4.12: Schematic diagram of current mirror and current source [21]. $\left(\frac{W}{L}\right)_{15}=\left(\frac{W}{L}\right)_{16}$, $\left(\frac{W}{L}\right)_{17}=\left(\frac{W}{L}\right)_{11}=\left(\frac{W}{L}\right)_{13}$.

A simple current mirror and a simple current source have been chosen as a current control for the level shifter. The schematic is shown in Figure 4.12 [21]. Transistor M17 is a simple current source that supply current to the current mirror. Transistors M15 and M16 comprise a simple current mirror. 
Transistor M16 is always in active mode. It governs the current rate of the level shifter. However, the current mirror has a low rejection to power supply variations.

Recall that

$$
\begin{aligned}
& \left(\frac{W}{L}\right)_{15}=\left(\frac{W}{L}\right)_{16} . \\
& \therefore v_{G S_{15}}=v_{G S_{16}}
\end{aligned}
$$

The drain current of M15 and M16 is

$$
I_{D_{16}}=I_{D_{15}}=\frac{K^{\prime}{ }_{L V P}}{2} \frac{W_{15}}{L_{15}} \operatorname{Vov}_{15}^{2}
$$

where overdrive voltage $\operatorname{Vov}_{15}=V_{G S_{15}}-V_{t_{L V P}}$. We rearrange the previous equation to find the width of M15:

$$
W_{15}=\frac{2 I_{D} L_{15}}{K_{L V P}^{\prime} \operatorname{Vov}_{15}{ }^{2}}
$$

An overdrive voltage of $0.45 \mathrm{~V}$ maintains M15 in saturation mode during operation. The estimated widths of M15 and M16 were $235.7 \mu m$. The widths of M15 and M16 were adjusted to $240 \mu m$ in the final design.

Transistor M17 is a simple current source for the current mirror. The aspect ratio of M17 is calculated with the equation below.

$$
\frac{W_{M 17}}{L_{e f f_{M 17}}}=\frac{-I_{D} 2\left(1+\theta\left(V_{G S}-V_{t}\right)\right)}{K^{\prime}\left(V_{G S}-V_{t}\right)}
$$

The estimated width of $W_{M 17}$ was $6.09 \mu \mathrm{m}$. It was adjusted to $7 \mu \mathrm{m}$ after simulation.

In the long term, a more sophisticated current source that does not depend on supply voltage and technological parameters should be used. For a simple current mirror, increasing the width of M15 reduces the minimum output voltage. Table 4.5 summarizes the final length and width of all transistors used in power stage and gate drivers. 
Table 4.5: Transistor dimensions in the final design.

\begin{tabular}{lcccc}
\hline Tr. no. & Instance no. & Transistor types & W $[\mu m]$ & L $[\mu m]$ \\
\hline M1 & M701 & HV PMOS & $100 \times 100$ & 100 \\
M2 & M702 & HV NMOS & $100 \times 100$ & 100 \\
M3 & M203 & HV PMOS & 250 & 4 \\
M4 & M204 & HV NMOS & 200 & 6 \\
\hline M9 & M209, M309 & HV NMOS & 70 & 6 \\
M10 & M210, M309 & HV NMOS & 70 & 6 \\
M11 & M211, M311 & LV PMOS & 7 & 0.8 \\
M12 & M212, M312 & LV PMOS & 7 & 0.8 \\
M13 & M213, M313 & LV PMOS & 7 & 0.8 \\
M14 & M214, M314 & LV PMOS & 7 & 0.8 \\
M15 & M215, M315 & LV NMOS & 240 & 0.8 \\
M16 & M216, M316 & LV NMOS & 240 & 0.8 \\
M17 & M217, M317 & LV PMOS & 7 & 0.8 \\
& M303 & HV PMOS & 381 & 4 \\
& M304 & HV NMOS & 280 & 6 \\
\hline
\end{tabular}

Notes:

Instance numbers of the form M7xx represent power transistors. Instance numbers of the form M2xx and M3xx are transistors used in the PMOS driver and NMOS driver respectively. In the table, the first column comprises the transistor numbers used in this chapter for design purposes. The second column contains the instance numbers that are employed in the schematic diagrams for simulation. All schematic diagrams for simulation can be found in Appendix D. 


\subsection{Digital Block}

A digital block is implemented with combinational logic to provide the functions of power stage enabling, faulty signal filtering and output buffering. It is located prior to the gate drivers of the amplifier. The schematic of the digital block is shown in Figure 4.13. It accepts a standard $5 \mathrm{~V}$ power supply, consisting of three inputs and two outputs. Three inputs are enable, PMOS signal and NMOS signal; denoted by the variables EN, $i n_{P}$ and $i n_{N}$. Two outputs are NMOS signal output and PMOS signal output; designated by $Y_{N}$ and $Y_{P}$. Outputs of the digital block are routed to the input of the level shifters. Each level shifter requires these signals and their complements for driving its transistors. Therefore, multiple stages of inverter are required to handle large capacitor loads. Dalsa's CMOS cell library manual [23] provides parameters and formula for calculating the propagation delay time of each stage.

$$
T_{p}[n s]=T_{\text {intrinsic }}[n s]+T_{\text {drive }}[n s / p F] \times C_{\text {load }}[p F]
$$

where $T_{p}$ denotes the propagation delay time of the respective stage; $T_{\text {intrinsic }}$ and $T_{\text {drive }}$ are parameters provided in Dalsa's CMOS cell library manual. The total propagation delay time of multiple stages is acquired by summing the delay time of each stage.

Enable pin is responsible for the on-off function of the power stage. When EN input is low, the circuitry locks up the power stage regardless of the inputs. This is done by biasing both power transistors in the cut off mode. When EN is high, outputs will follow the respective inputs, except when a faulty state $\left\{i n_{P}, i n_{N}\right\}=\{0,1\}$ is identified. This state will turns both power transistors on together and cause permanent damage to them. The combinational logic was implemented with the digital library offered in the design kit. Table 4.6 is the truth table of the digital block.

On the left hand side of the truth table, under the input variables, are eight rows of all possible combinations of 1's and 0's that these variables may have. The first four rows (enable EN $=0$ ) indicate that the amplifier is working in standby mode. Therefore, output variable $Y_{P}$ and $Y_{N}$ assign logic high and logic low respectively for biasing power transistors in the cut off mode. For rows five, seven and eight, outputs follow the respective inputs. The input of the sixth row overrides, because it eventually creates a short by turning on both power transistors.

The simplified Boolean function for the two outputs $Y_{P}$ and $Y_{N}$ can be derived by solving the Kar- 
Table 4.6: Truth Table.

\begin{tabular}{|c|c|c|c|c|c|c|c|}
\hline & $\mathrm{EN}$ & $i n_{P}$ & $i n_{N}$ & $Y_{P}(1)$ & $Y_{N}(1)$ & PMOS & NMOS \\
\hline & 0 & 0 & 0 & 1 & 0 & off & off \\
\hline & 0 & 0 & 1 & 1 & 0 & off & off \\
\hline & 0 & 1 & 0 & 1 & 0 & off & off \\
\hline & 0 & 1 & 1 & 1 & 0 & off & off \\
\hline & 1 & 0 & 0 & 0 & 0 & on & off \\
\hline \multirow[t]{3}{*}{ (2) } & 1 & 0 & 1 & 1 & 0 & off & off \\
\hline & 1 & 1 & 0 & 1 & 0 & off & off \\
\hline & 1 & 1 & 1 & 1 & 1 & off & on \\
\hline
\end{tabular}

Note 1:

$Y_{P}$ is active low and $Y_{N}$ is active high.

Note 2:

The state $i n_{P}=0$ and $i n_{N}=1$ will turn on both power transistors; therefore, combinational circuit switches them off for protection.

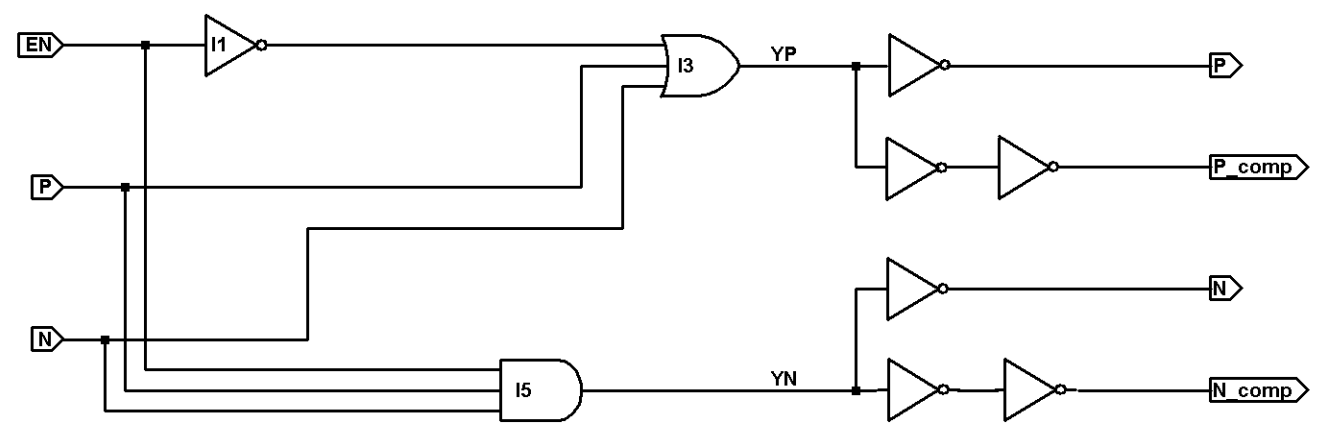

Figure 4.13: Schematic diagram of the digital block. 
naugh Maps shown in Figure 4.14(a) and (b). The corresponding simplified sum of product expressions are listed directly below each map.

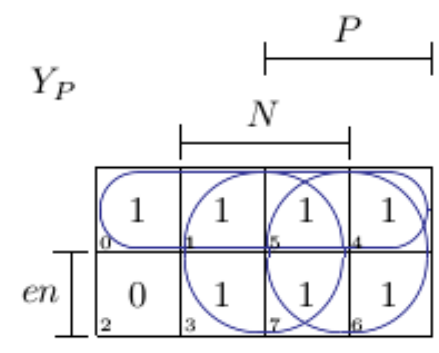

$$
Y_{P}=\overline{E N}+P+N
$$

(a)

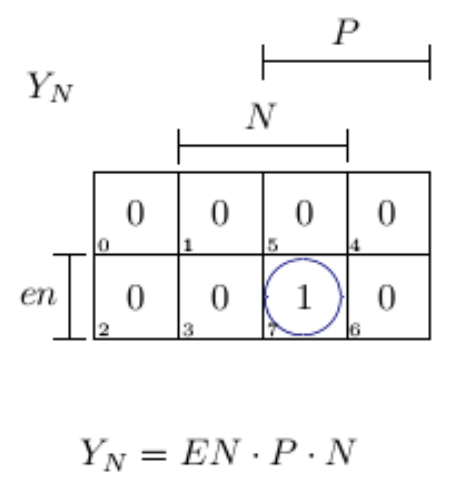

(b)

Figure 4.14: Map for input functions and output of the digital block. (a) Functions of PMOS (b) Functions of NMOS. 


\section{Chapter 5}

\section{Simulation Results}

\subsection{Pre-layout simulations}

The performance of the proposed integrated amplifier has been appraised with various configurations in order to establish a comprehensive solution that demonstrates an acceptable level of third harmonic and efficiency. Figure 5.1 and Figure 5.2 show the output voltage $v_{D S_{M 702}}$ and the power transistors'

drain current $I_{D_{M 701}}$ and $I_{D_{M 702}}$ waveforms in time domain at steady state. Since the output stage is directly connected to an ultrasound transducer instead of through a tuned network, the class DE amplifier does not operate at its optimum working condition. This can be identified when a small trough appears at the rising or falling edge of the $v_{D S_{M 702}}$ waveforms in Figure 5.1. Figure 5.3 illustrates the Discrete Fourier Transform DFT of the output voltage waveform at steady state. Spectrum was obtained using the DFT function of Calculator in the Cadence's Analog Design Environment. The simulation was run for $100 \mu s$; one hundred periods were generated. The time period for DFT was taken from $50 \mu s$ to $100 \mu s$.

Figure 5.4 plots the ratio of third harmonic to fundamental for the voltage waveform, as a function of duty ratio with a varying values of external capacitance at steady state. The vertical axis of the Figure is the percentage of magnitude of 3rd harmonic to the magnitude of the fundamental. The horizontal axis represents the duty ratio D of the control signals. In other words, duty ratio D excludes delays imposed by the combinational circuit, gate drivers and power transistors. Therefore, the turn-on duration measured at the output is longer than the turn-on duration measured at the input. An input pulse with 0.19 duty ratio eventually creates an output pulse with a duty ratio of 0.25 . From the figure, the third harmonic becomes lowest when the duty ratio D was between 0.22 and 0.23 at steady state. 


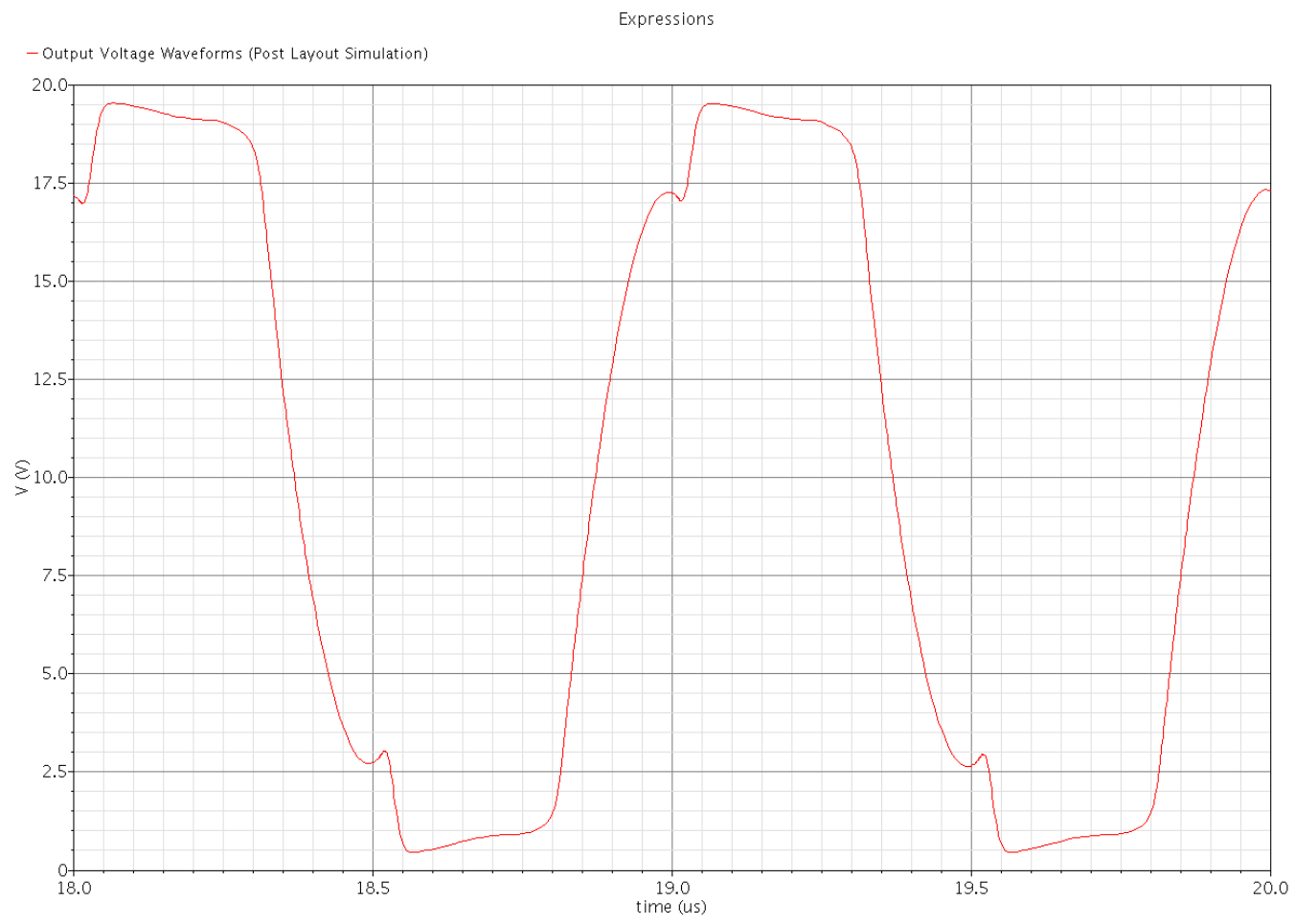

Figure 5.1: Amplifier output voltage waveforms versus time at steady state. $\mathrm{f}=1 \mathrm{MHz}, \mathrm{D}=0.22$ and $C_{\text {ext }}=0 p F$.

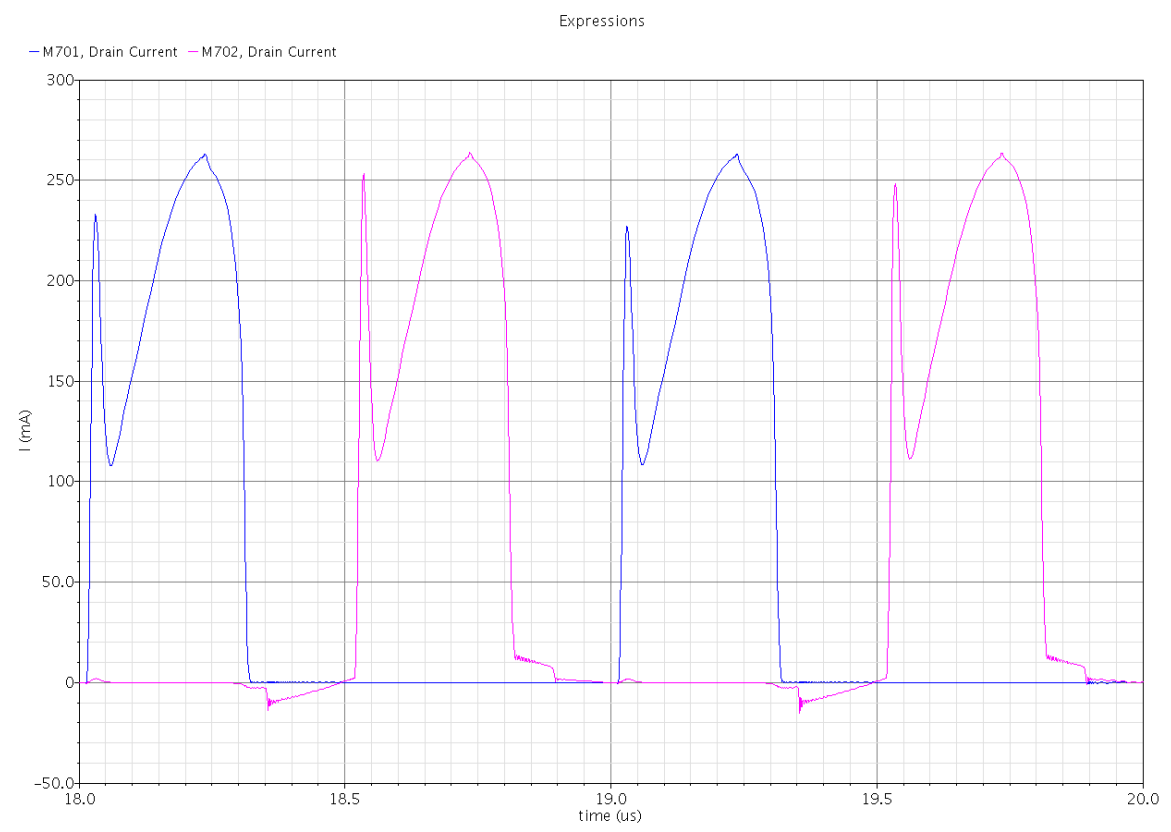

Figure 5.2: Drain current waveforms of M701 and M702 against time at steady state. 


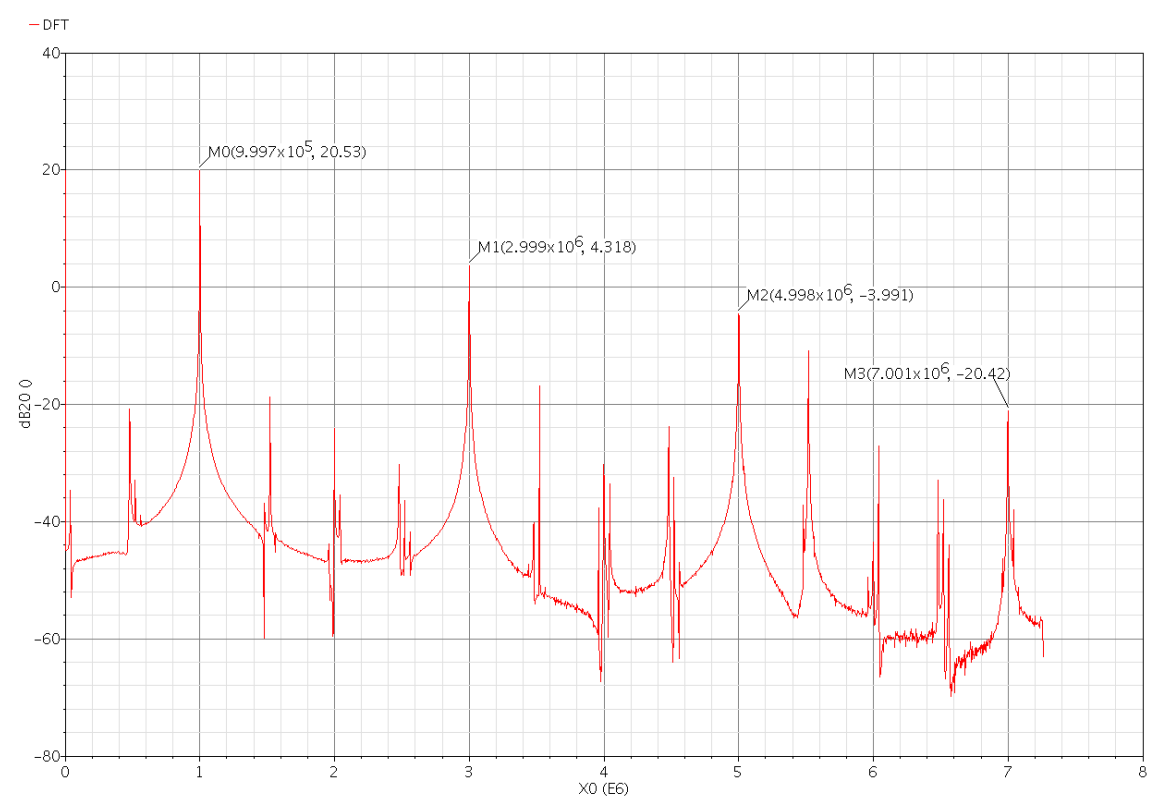

Figure 5.3: Discrete Fourier Transform of the amplifier's output voltage waveforms at steady state.

The third harmonic is approximately $18 \%$ of the fundamental without any external capacitance and it is below $5 \%$ with an external capacitance of $1 \mathrm{nF}$. High current spikes are generated when the amplifier starts. Increasing the external capacitance will increase the magnitude of current spikes and the width of the power supply lines; therefore, the maximum capacitance of the external capacitor is limited to $1 \mathrm{nF}$. In addition, an external capacitance of more than $1 \mathrm{nF}$ will bring the efficiency down below $80 \%$.

Figure 5.5 plots the steady state efficiency versus switch-on duty ratio D for a varying external capacitance. As the external capacitance increases, the driver efficiency decreases and the gaps between curves become wider. Depending on the external capacitance, the point of maximum efficiency occurs in between $D=0.24$ and $D=0.27$. In general, the efficiency of the proposed design is above $80 \%$ within the configurations that have been tested. The amplifier efficiency is higher without the external capacitance because the switching loss is lower.

Figure 5.6 illustrates an overview of amplifier's output power to $R_{\mathrm{s} 1}$ against switch-on duty ratio D for a varying value of external capacitance. Output power is defined as the power consumption of the resistor $R_{s 1}$, the series resistor in the fundamental branch in the ultrasound transducer model, which represents the energy delivery to the acoustic power field, plus mechanical losses at fundamental 


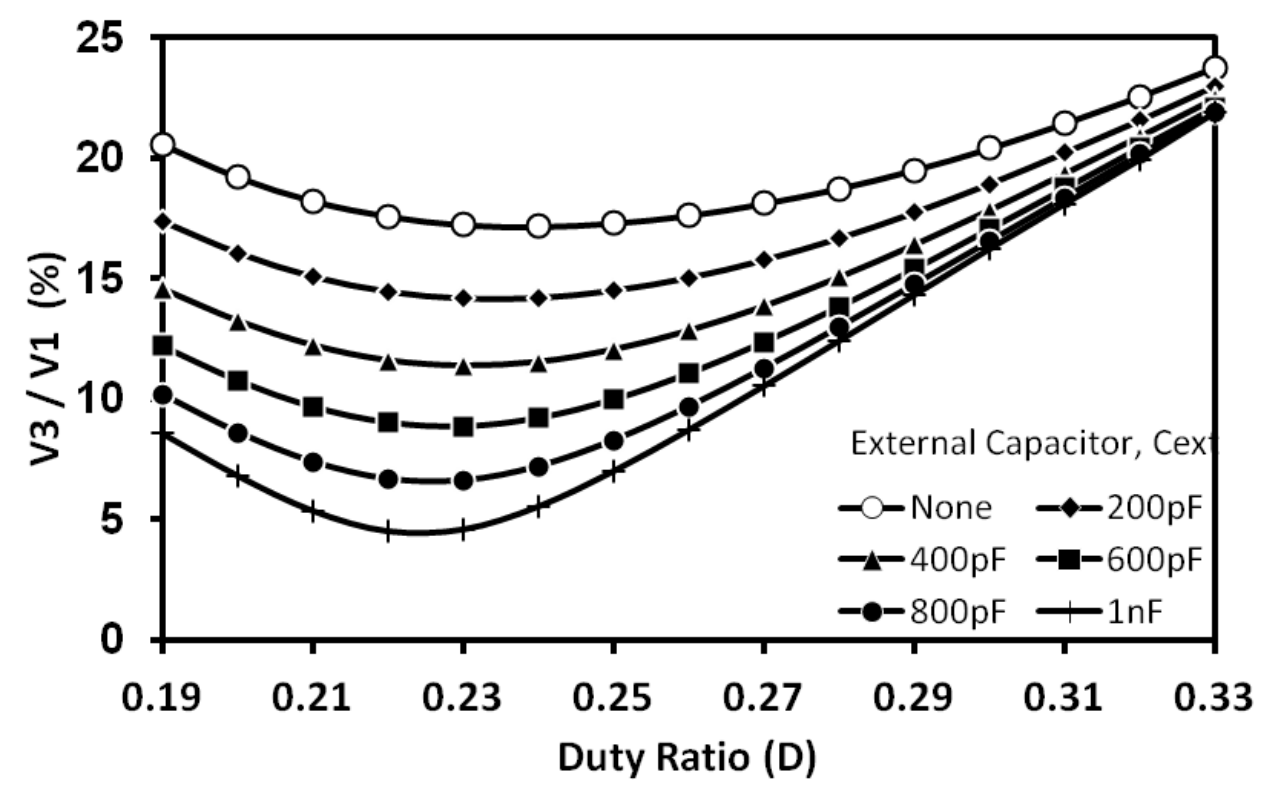

Figure 5.4: Percentage of third harmonic to fundamental of the amplifier output waveform as a function of switch-on duty ratio D with varying external capacitance at steady state.

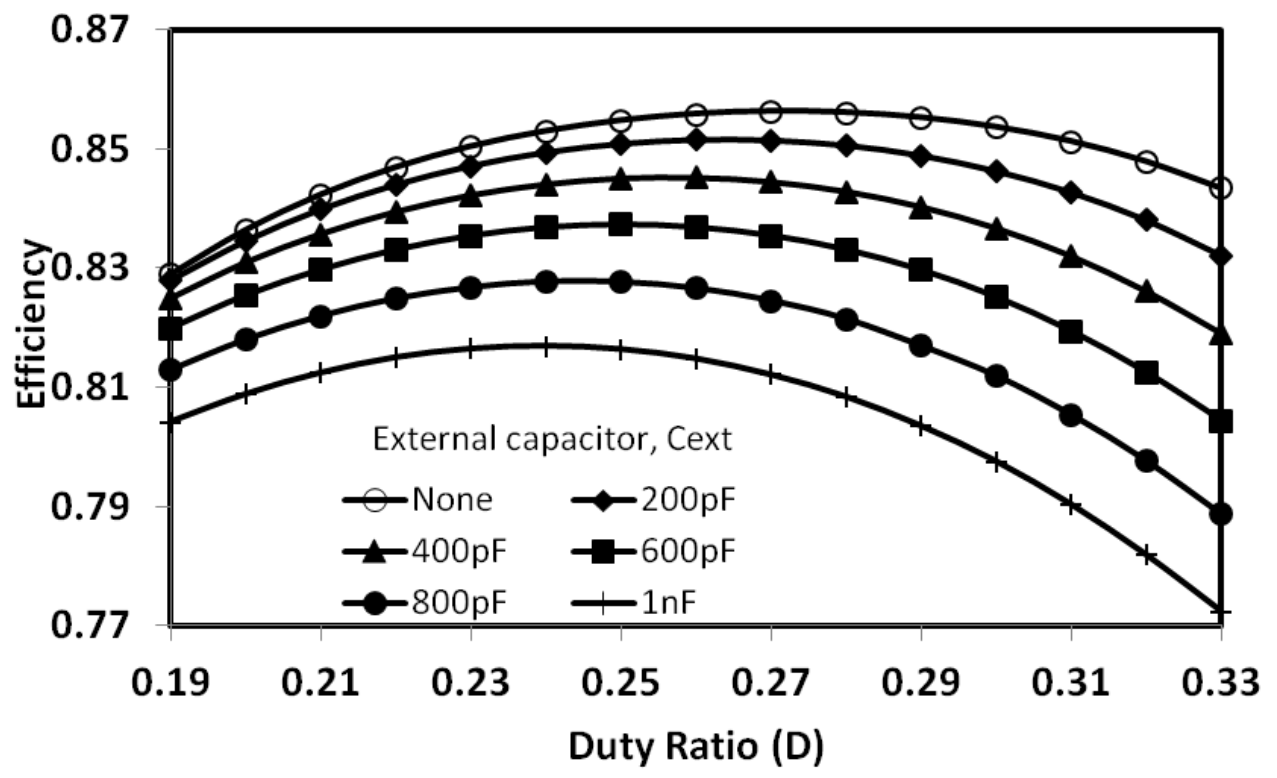

Figure 5.5: Efficiency versus switch-on duty ratio D with a varying external capacitance at steady state. 
frequency. Increasing the external capacitance reduces the output power of the amplifier. This effect becomes more noticeable at a lower switch-on duty ratio due to the increasing of switching loss. The amplifier produces higher power as duty ratio increases.

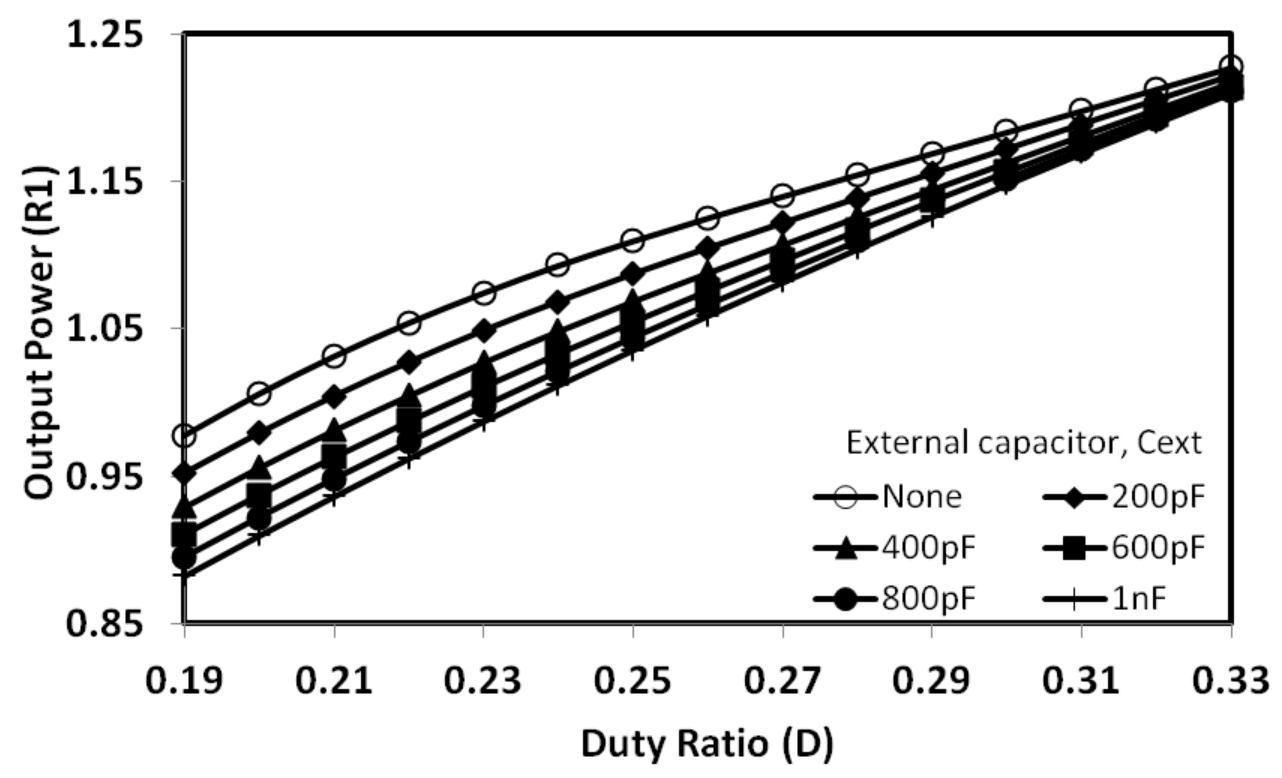

Figure 5.6: Output power against switch-on duty ratio with a varying external capacitance at steady state.

\subsection{Post-layout simulations}

Post layout simulation extracts parasitic capacitance that might affect the performance of the original design in a layout. A layout for the integrated amplifier was designed and its performance was verified using Virtuoso $(\mathbb{B}$ ) and Spectre $($. Figure 5.7 depicts the completed layout view of the die, which occupies an area of $2.5 \mathrm{~mm} \times 1.6 \mathrm{~mm}$. The function of each pad is also identified in the same figure. The middle-right area is occupied by power transistors. The outer frame that surrounds the die consists of the input-output pads. Gate drivers and the digital block are located on the left. The layout design follows the best practices recommended in [24]. A floorplan has been made before the actual layout design commenced. Individual cell layout was verified with the corresponding cell schematic diagram. Each cell occupies the minimum possible area. Transistors are surrounded by metal shields that facilitate latch-up protection. Cell blocks and digital gates are arranged discreetly for shortest critical path connections. Sufficient number of contacts and vias are used to ensure current 
requirements are met. Extra contacts and vias are used in all metal-to-polysilicon and metal-to-metal connections to ameliorate electromigration. Figures 5.8 and 5.9 compare both the pre- and post-layout simulation results for output voltage waveform and output current waveform to the transducer with time domain.

Individual layout view of the integrated amplifier can be found in Appendix E. 


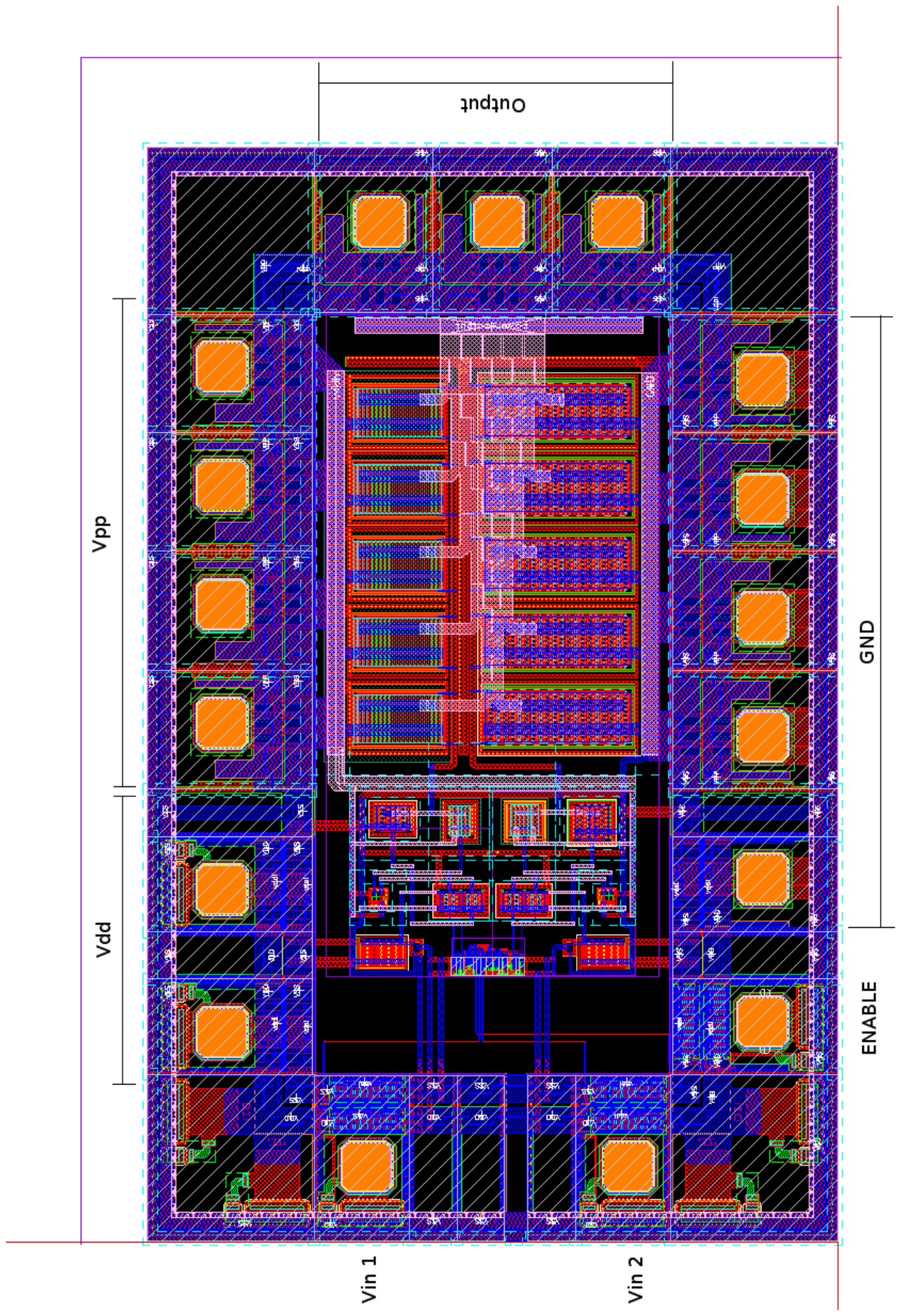

Figure 5.7: Layout of the integrated amplifier. 


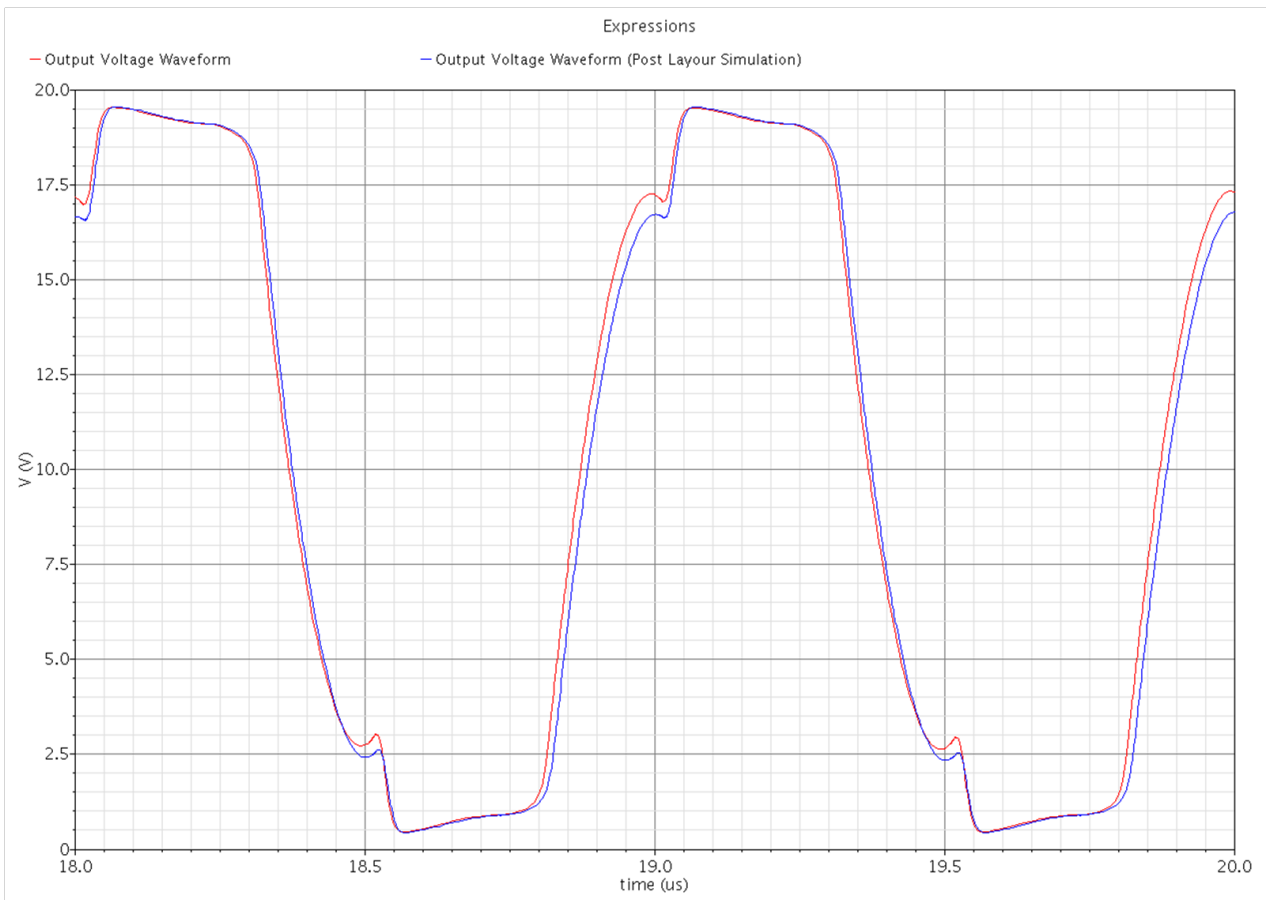

Figure 5.8: Output voltage waveforms of pre- and post-layout simulations.

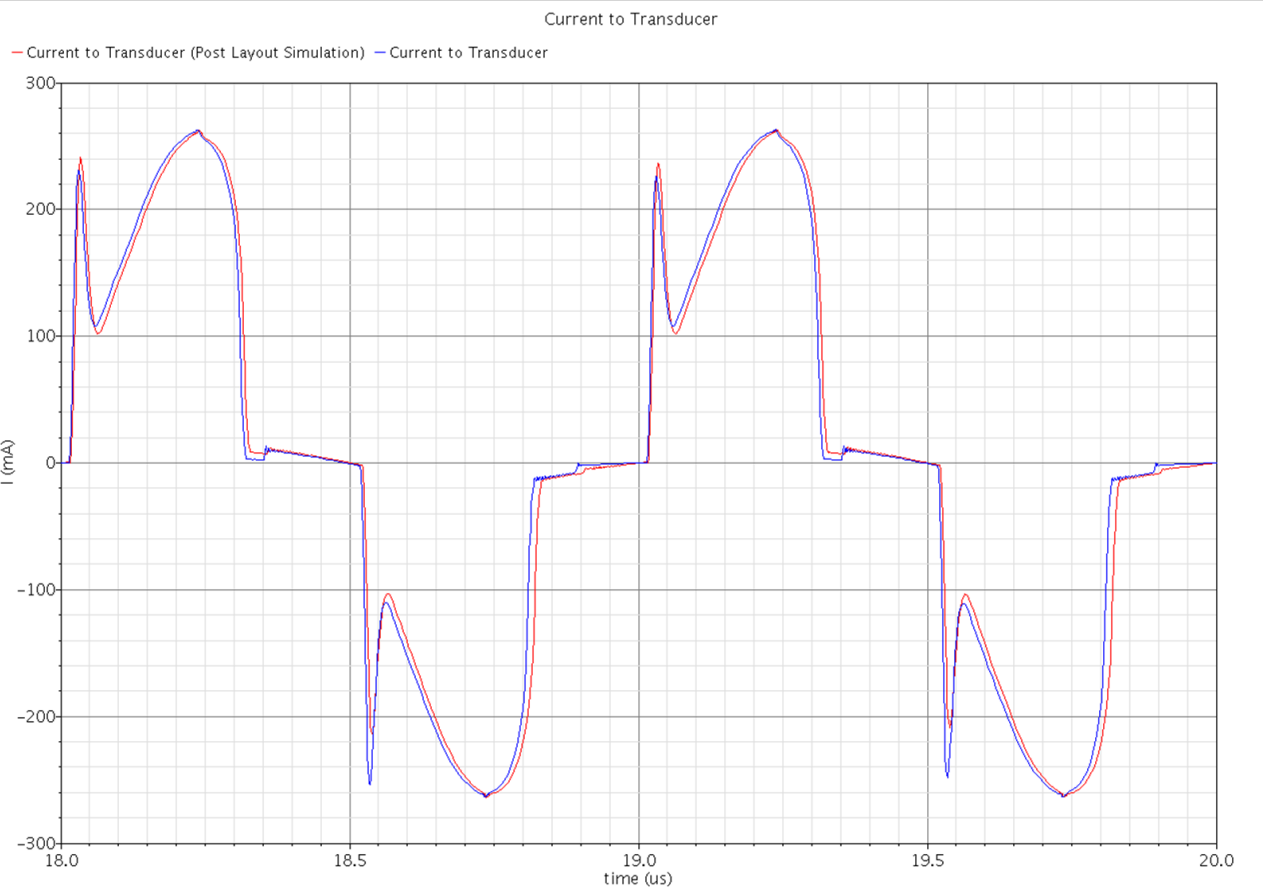

Figure 5.9: Output current waveforms of pre- and post-layout simulations. 


\section{Chapter 6}

\section{Conclusions}

The objective of this thesis was to design a CMOS power amplifier for a piezoelectric transducer to be installed near the piezoelectric resonator inside an MRI environment. Several of the challenges of designing the integrated amplifier have been addressed:

1. Each amplifier should occupy a minimum possible area: an effort was made to reduce the chip area by avoiding the use of on-chip resistors, inductors and capacitors. Also, transistors were designed to be as small as possible. The overall area of the amplifier is $2.5 \mathrm{~mm}$ by $1.6 \mathrm{~mm}$, which is smaller than any other design reported in the literature, such as [13]. Without the pads, the amplifier occupies an area of $0.9 \mathrm{~mm}^{2}$.

2. The use of magnetic components, such as inductors, has been avoided because they can interfere with the MRIs reception and distort the image [3].

3. The amplifier is efficient: overall efficiency is $80 \%$. This, combined with the low chip area, will enable the integration of several amplifiers in a single chip.

4. The proposed amplifier is capable of delivering $1 \mathbf{W}$ of power to the load at $1 \mathrm{MHz}$. The third harmonic level is below $20 \%$ on the electrical side in the worst case. The third harmonic can be reduced at the cost of some efficiency by increasing the value of the external capacitor. The expected third harmonic level in the acoustic pressure field should be approximately $9.5 \mathrm{~dB}$ below the electrical level according to the measurements reported in Chapter 3. Figure 6.1 illustrates the projected percentage of the acoustic third harmonic to fundamental as a function of switchon duty ratio $\mathrm{D}$, with a varying external capacitance based on the results of circuit simulations, 
combined with the characterization of the transducer provided in Chapter 3. It shows that the maximum ratio of third harmonic to fundamental of the proposed integrated amplifier is below $9 \%$ in the acoustic pressure field.

The proposed integrated amplifier will enable the implementation of more compact and less expensive multi-element HIFU equipment with MRI guidance. Unlike other published designs [2], [7] - [11], [12], the amplifier is MRI compatible because it does not require any external inductor.

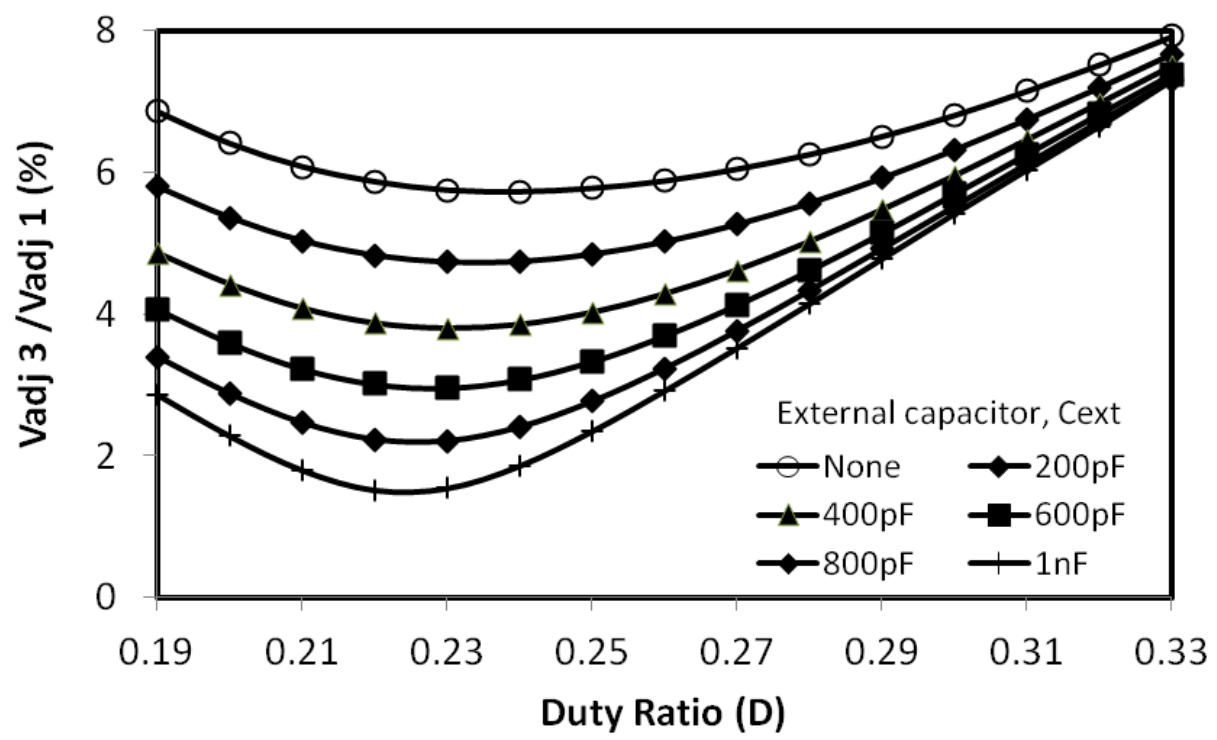

Figure 6.1: Projected percentage of third harmonic to fundamental in the acoustic pressure waveform as a function of switch-on duty ratio D with varying external capacitance.

\subsection{Future Work}

The fabrication cost of the Teledyne-DALSA $0.8 \mu \mathrm{m}$ 5/20V CMOS process has recently increased because of low demand. Thus, the design has to be re-implemented in another CMOS process from Austria Microsystems, AMS $0.35 \mu \mathrm{m}$. The fabricated chip will be tested with the piezoelectric resonator to verify the harmonics in the acoustic field and to confirm its usability in the MRI.

It is recommended that a current sensing circuit be included to ensure that the power transistors are working at minimum power dissipation, because the amplifier is very sensitive to the input duty ratio. This can be done by detecting current spikes generated by the power transistors during an off-to-on transition. 


\section{Appendix A}

\section{Transducer Housing}

The housing is made of PVC. A piezoelectric resonator is placed at the opening of the housing. Epoxy is used to fill up the $1.25 \mathrm{~mm}$ gap between the circumference of piezoelectric resonator and inner circumference of the housing. A $3 \mathrm{~mm}$ hole is located on the wall of the housing for the coax to come through and three $1 \mathrm{~mm}$ holes are located on the top of the housing to grounding the connection for the piezoelectric resonator.

\section{Assembly instructions}

First, three ground wires are soldered on the top, near the edge of the piezoelectric resonator. Then, the centre conductor of the coax is connected to another three short wires that are soldered to bottom of the piezoelectric resonator. Next, the piezoelectric resonator and the housing are glued together with epoxy. Finally, a BNC connector is installed to the other end of the coax cable. 
Figure A.1: Transducer Housing

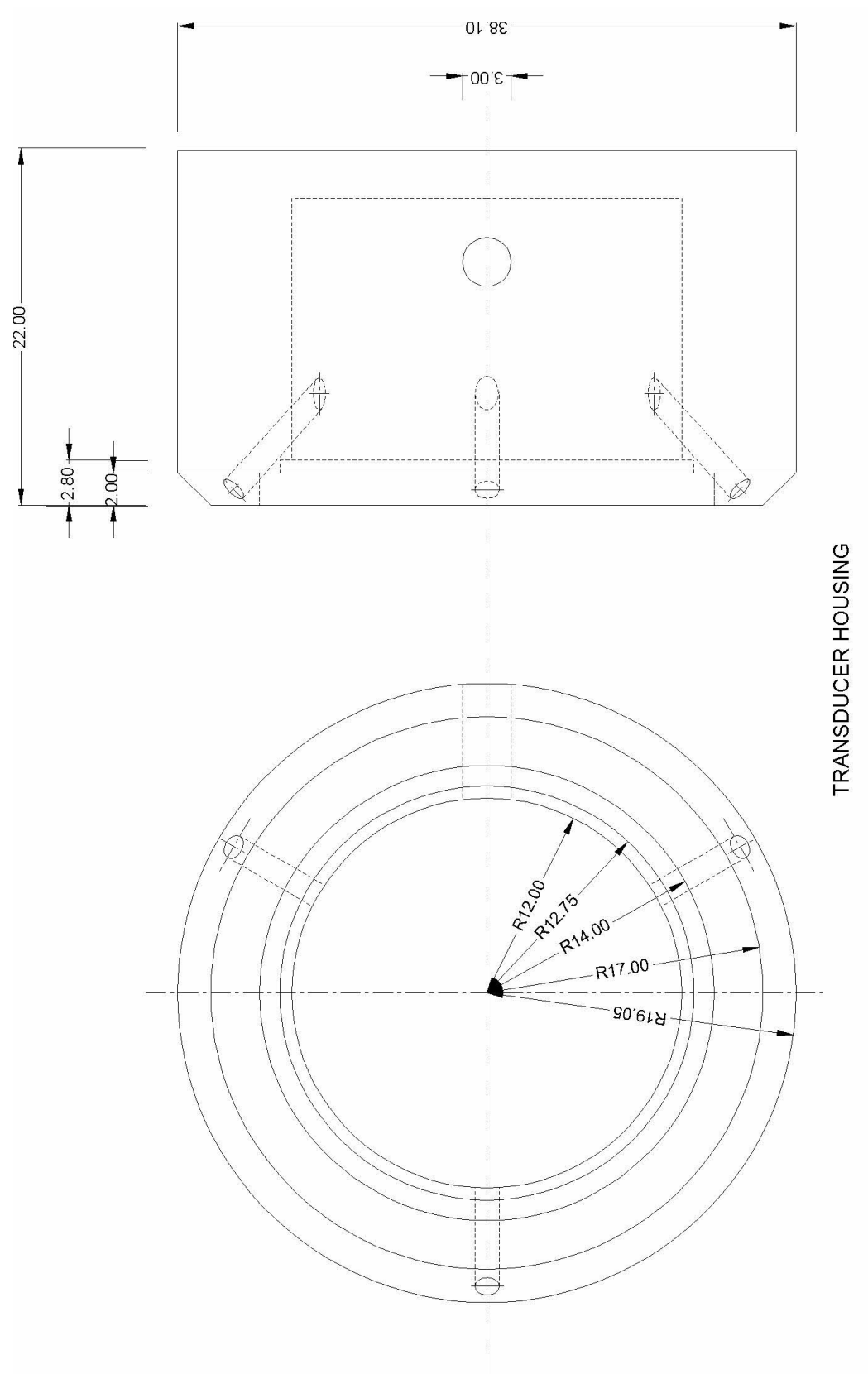




\section{Appendix B}

\section{Results of the Attenuation of}

\section{Harmonics with $20 V_{p-p}$ Sinusoidal Signals}

This appendix contains the results of the attenuation of harmonics when the transducer was excited with $20 V_{p-p}$ sinusoidal signals. The values in the last column in Table B.1 denote the attenuation between the voltage applied on the piezoelectric plate and the measurement by hydrophone, with adjustment. The difference of attenuation between $1 \mathrm{MHz}$ and $5 \mathrm{MHz}$ was approximately $18.62 \mathrm{dBV}$, which generally agrees with the $2 \mathrm{~V}$ test. However, the difference of attenuation between $1 \mathrm{MHz}$ and $3 \mathrm{MHz}$ was $2.76 \mathrm{dBV}$, which is lower than the result of the $2 \mathrm{~V}$ test given in Chapter 3, Table 3.1. This inconsistency may indicate that a mistake was made while the experiment was conducted; another experiment should be performed to clarify the confusion.

Table B.1: Attenuation of harmonics with $20 V_{p-p}$ sinusoidal signals.

\begin{tabular}{ccccc}
\hline $\mathrm{f}(\mathrm{MHz})$ & $V_{g}$ & $V_{\text {in }}$ & $V_{\text {measured }}$ & Attenuation \\
\hline 1 & $20.01 \mathrm{~V}$ & $11.62 \mathrm{~V}$ & $250 \mathrm{mV}$ & $-32.48 \mathrm{dBV}$ \\
3 & $20.01 \mathrm{~V}$ & $5.110 \mathrm{~V}$ & $250 \mathrm{mV}$ & $-35.25 \mathrm{dBV}$ \\
5 & $20.01 \mathrm{~V}$ & $2.050 \mathrm{~V}$ & $58.0 \mathrm{mV}$ & $-51.10 \mathrm{dBV}$ \\
\hline
\end{tabular}




\section{Appendix C}

\section{An Estimation of M4's width using the Differential Equation Method}

Suppose that the output of the gate driver is connected to a capacitive load $C$ that is equivalent to the gate capacitor of M1. The gate width of the transistor can be estimated by the differential equation method [22]. When $C$ has been fully charged to supply voltage $V p p$ for a while, M4 is turned on. It soon enters into saturation mode. The rate of change of the capacitor voltage is defined as follows:

$$
C \frac{d V}{d t}=-i_{D_{M 4}}
$$

Substituting the saturation current expression for $i_{D_{M 4}}[22]$, we get

$$
-C \frac{d V}{d t}=\frac{K^{\prime}}{2} \frac{W}{L}\left(V_{G S}-V_{t}\right)^{2} \frac{1}{1+\theta\left(V_{G S}-V_{t}\right)},
$$

Integrating both sides,

$$
-C \int_{\mathrm{V} 90 \%}^{\mathrm{Vov}} d V=\int_{0}^{\tau} d t \frac{K^{\prime}}{2} \frac{W}{L}\left(V_{G S}-V_{t}\right)^{2} \frac{1}{1+\theta\left(V_{G S}-V_{t}\right)},
$$

where $\tau$ represents the moment that M4 transfers from saturation mode to triode mode; Vov stands for overdrive voltage or $\left(V_{G S}-V_{t}\right)$ for $\mathrm{M} 4$, and $V 90 \%$ is $90 \%$ of $V p p$. Evaluating the the first integral gives us

$$
\int_{\mathrm{V} 90 \%}^{\mathrm{Vov}} d V=V o v-0.9 V p p=V_{s a t}
$$

The second integral is given by:

$$
\int_{0}^{\tau} d t=\tau
$$


Substituting $V_{\text {sat }}$ and $\tau$ into Equation (C.1), we have,

$$
\frac{-C \cdot V_{s a t}}{\tau}=\frac{K^{\prime}}{2} \frac{W}{L}\left(V_{G S}-V_{t}\right)^{2} \frac{1}{1+\theta\left(V_{G S}-V_{t}\right)} .
$$

At time $\tau$, M4 changes its mode from saturation to triode. The rate of change of the capacitor voltage is expressed as follows:

$$
-C \frac{d V}{d t}=\frac{K^{\prime}}{2} \frac{W}{L}\left(2\left(V_{G S}-V_{t}\right) V_{D S}-V_{D S}^{2}\right) \frac{1}{1+\theta\left(V_{G S}-V_{t}\right)} .
$$

Rearranging the above expression, we have

$$
\frac{d V}{\left(2\left(V_{G S}-V_{t}\right) V_{D S}-V_{D S}{ }^{2}\right)}=d t \frac{K^{\prime}}{-C 2} \frac{W}{L} \frac{1}{1+\theta\left(V_{G S}-V_{t}\right)} .
$$

Integrating both sides, it becomes

$$
\int_{\mathrm{Vov}}^{\mathrm{V} 10 \%} \frac{d V}{\left(2\left(V_{G S}-V_{t}\right) V_{D S}-V_{D S}{ }^{2}\right)}=\left(\int_{\tau}^{t_{f a l l}} d t\right)\left(\frac{K^{\prime}}{-C 2} \frac{W}{L} \frac{1}{1+\theta\left(V_{G S}-V_{t}\right)}\right),
$$

where $\operatorname{Vov}_{10 \%}$ is $10 \%$ of the supply voltage Vpp. Let $V_{t r i}$ be a solution to the integral on the left hand side of Equation (C.3), which can be simplified as follows:

$$
\begin{aligned}
V_{t r i} & =\int_{\mathrm{Vov}}^{\mathrm{V} 10 \%} \frac{d V}{\left(2\left(V_{G S}-V_{t}\right) V_{D S}-V_{D S}{ }^{2}\right)} \\
& =\left[-\frac{\ln \left(V_{D S}\right)}{2\left(V_{G S}-V_{t}\right)}+\frac{\ln \left[-2 V_{G S}+2 V_{t}+V_{D S}\right]}{2\left(V_{G S}-V_{t}\right)}\right]_{\mathrm{Vov}}^{\mathrm{V} 10 \%}
\end{aligned}
$$

The second integral of Equation (C.3) is

$$
\int_{\tau}^{t_{f a l l}} d t=t_{\text {fall }}-\tau
$$

Substituting $V_{t r i}$ and $\tau$ into Equation (C.3), it becomes

$$
V_{t r i}=-\frac{t_{f a l l}-\tau}{C}\left(\frac{K^{\prime}}{2} \frac{W}{L} \frac{1}{1+\theta\left(V_{G S}-V_{t}\right)}\right) .
$$

Combining Equation (C.2) and (C.4), we solve for the expression for the width:

$W=\frac{2 C L\left(-V_{s a t}-V_{s a t} \theta V_{G S}+V_{s a t} \theta V_{t}-V_{t r i} V_{G S}^{2}+2 V_{t r i} V_{G S} V_{t}-V_{t r i} V_{t}^{2}-V_{t r i} \theta V_{G S}{ }^{3}+3 V_{t r i} \theta V_{t}-3 V_{t r i} \theta V_{G S} V_{t}^{2}+V_{t r i} \theta V_{t}^{3}\right)}{t_{f a l l} K^{\prime}\left(V_{G S}{ }^{2}-2 V_{G S} V_{t}+V_{t}^{2}\right)}$

where overdrive voltage $\operatorname{Vov}=V_{G S}-V_{t}$. The first integral in Equation (C.1) is

$$
V_{\text {sat }}=\int_{\mathrm{V} 90 \%}^{\mathrm{Vov}} d V V .
$$


The first integral in Equation (C.3) is evaluated as follows.

$$
V_{t r i}=\int_{\text {Vov }}^{\mathrm{V} 10 \%} \frac{d V}{\left(2\left(V_{G S}-V_{t}\right) V_{D S}-V_{D S}^{2}\right)} V^{-2}
$$

Applying Equation (C.5), the width of M4 is calculated as follows:

$$
W_{M 4}=\frac{2 C L\left(-V_{s a t}-V_{s a t} \theta V_{G S}+V_{s a t} \theta V_{t}-V_{t r i}\left(V_{G S}\right)^{2}+2 V_{t r i} V_{G S} V_{t}-V_{t r i} V_{t}^{2}-V_{t r i} \theta\left(V_{G S}\right)^{3}+\cdots\right.}{t_{f a l l} K^{\prime}\left(V_{G S}{ }^{2}-2 V_{G S} V_{t}+V_{t}^{2}\right)} .
$$

The estimated width of M4 was $197.2 \mu \mathrm{m}$. 
Appendix D

\section{Schematic Diagram}

Schematic diagrams used in Spectre® for simulations. 
Figure D.1: Schematic diagram for simulating the integrated amplifier.

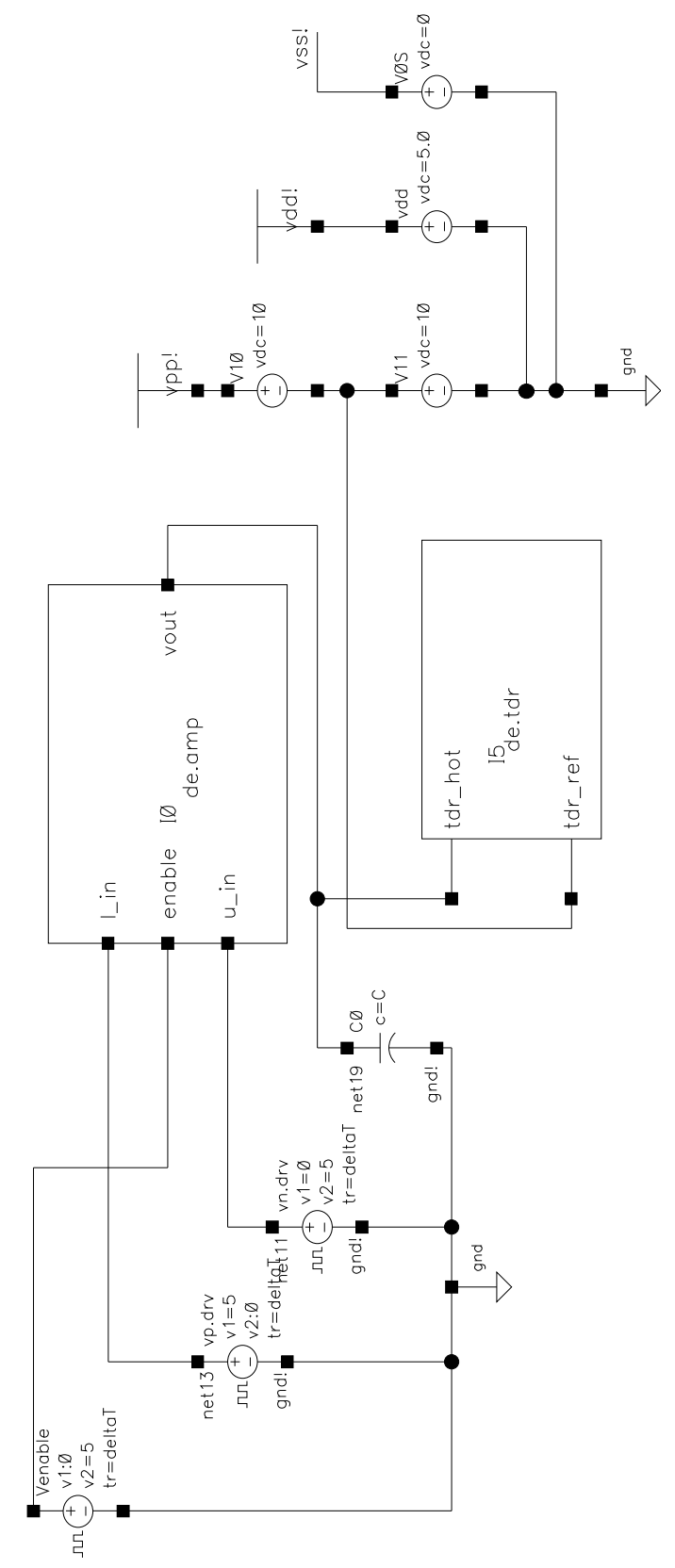


Figure D.2: Top level schematic of the integrated amplifier.

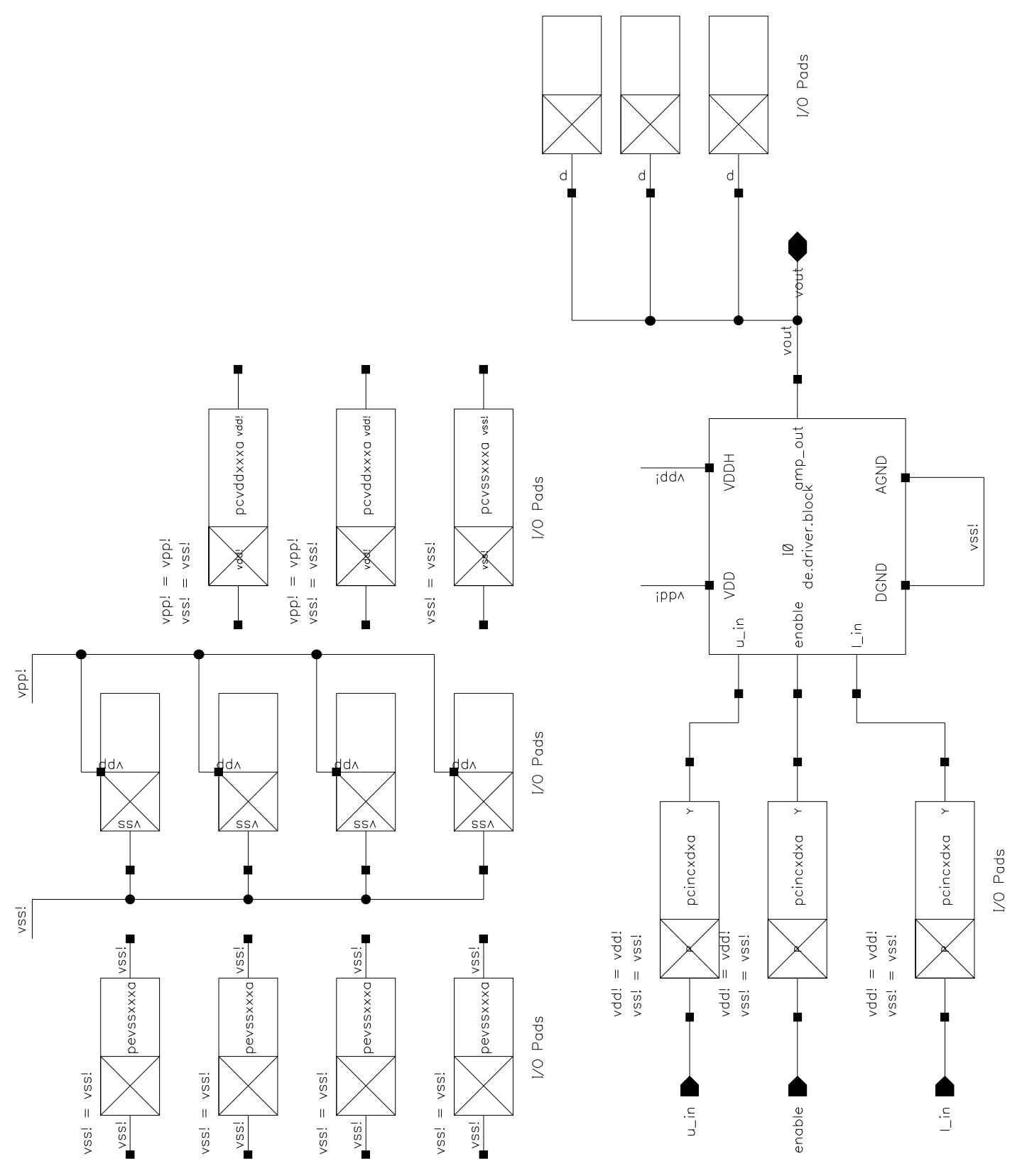


Figure D.3: Block diagram of the integrated amplifier.

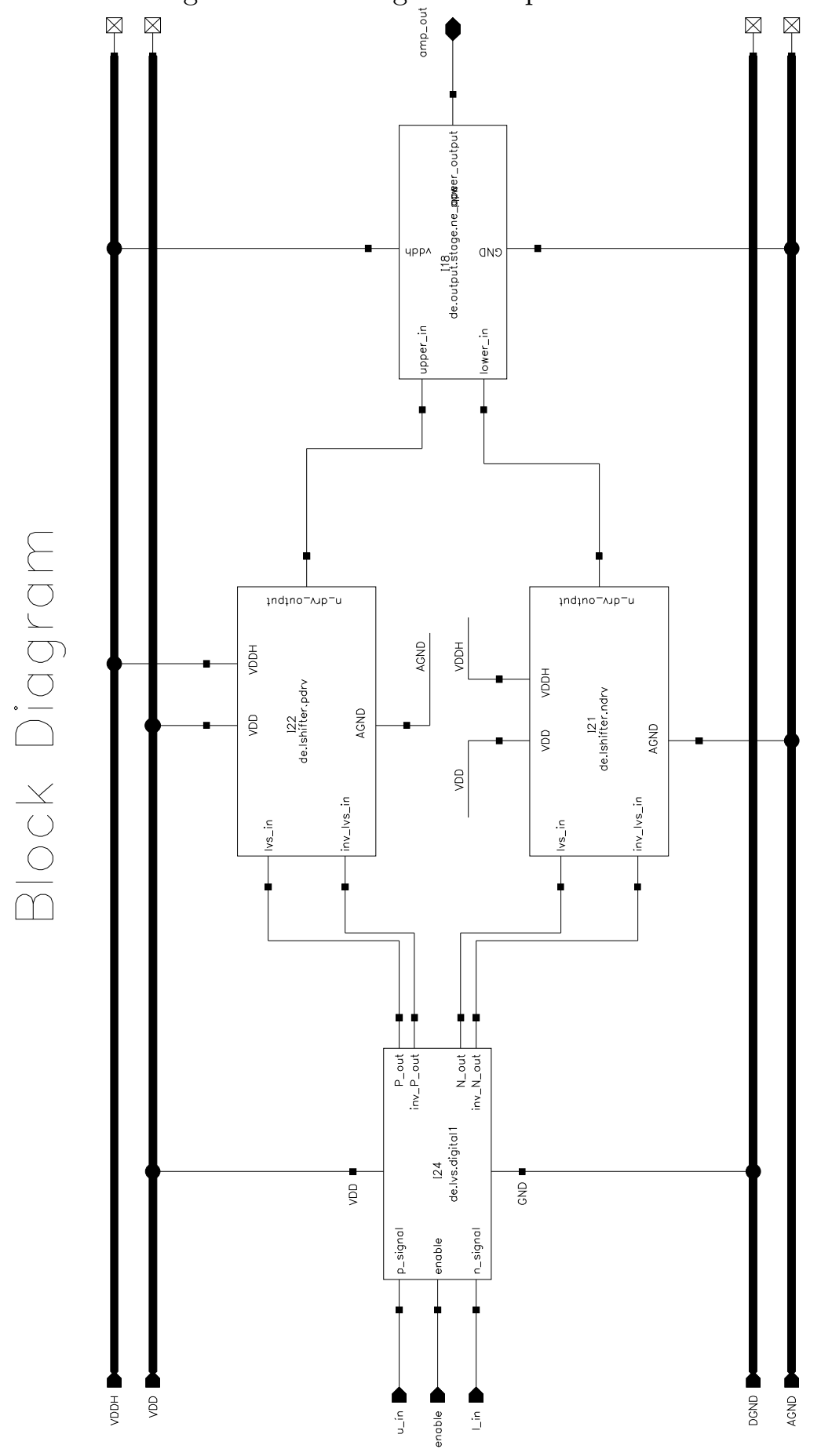


Figure D.4: Block diagram of M701 gate driver.

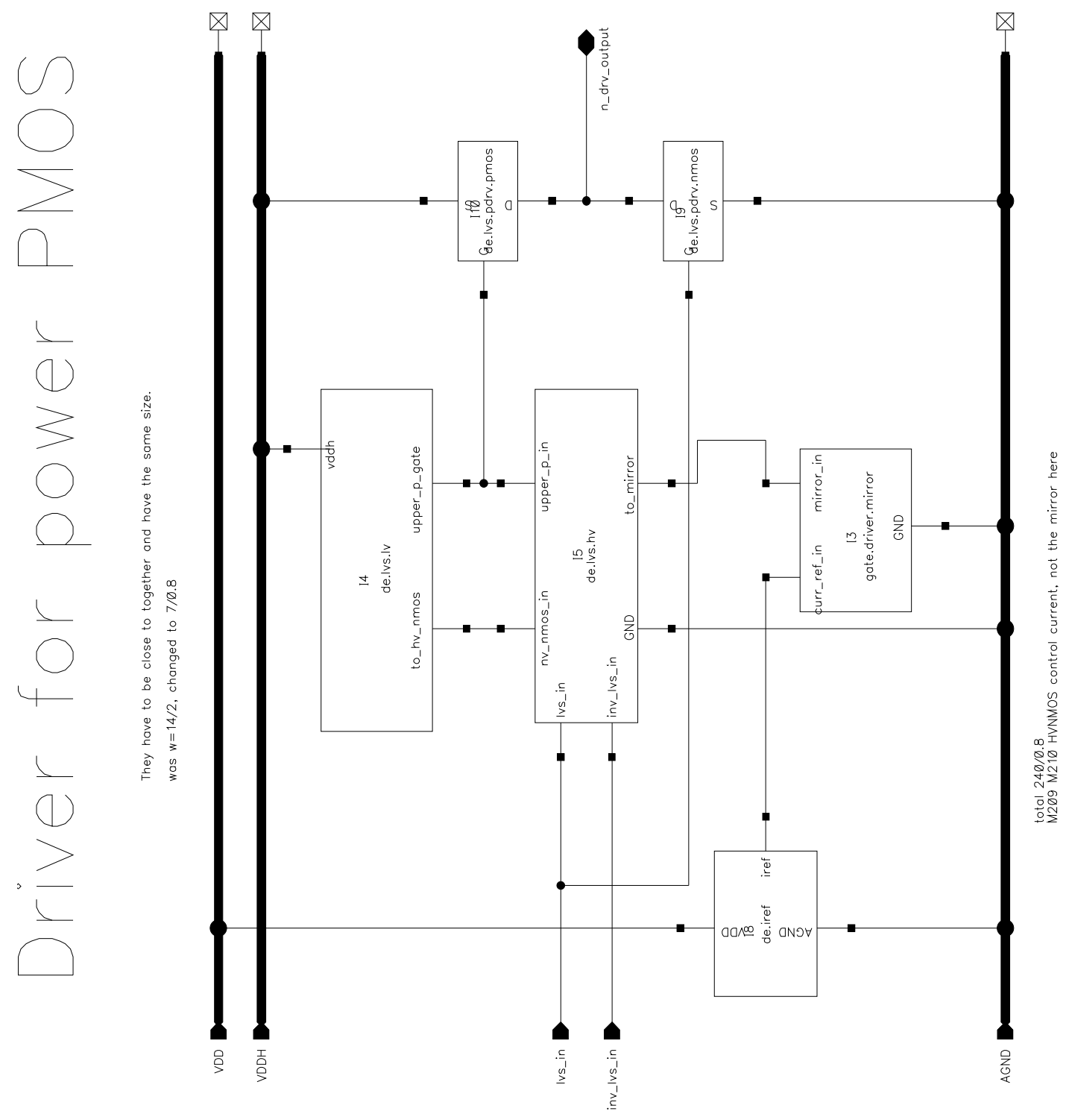


Figure D.5: Schematic diagram of M701 gate driver.

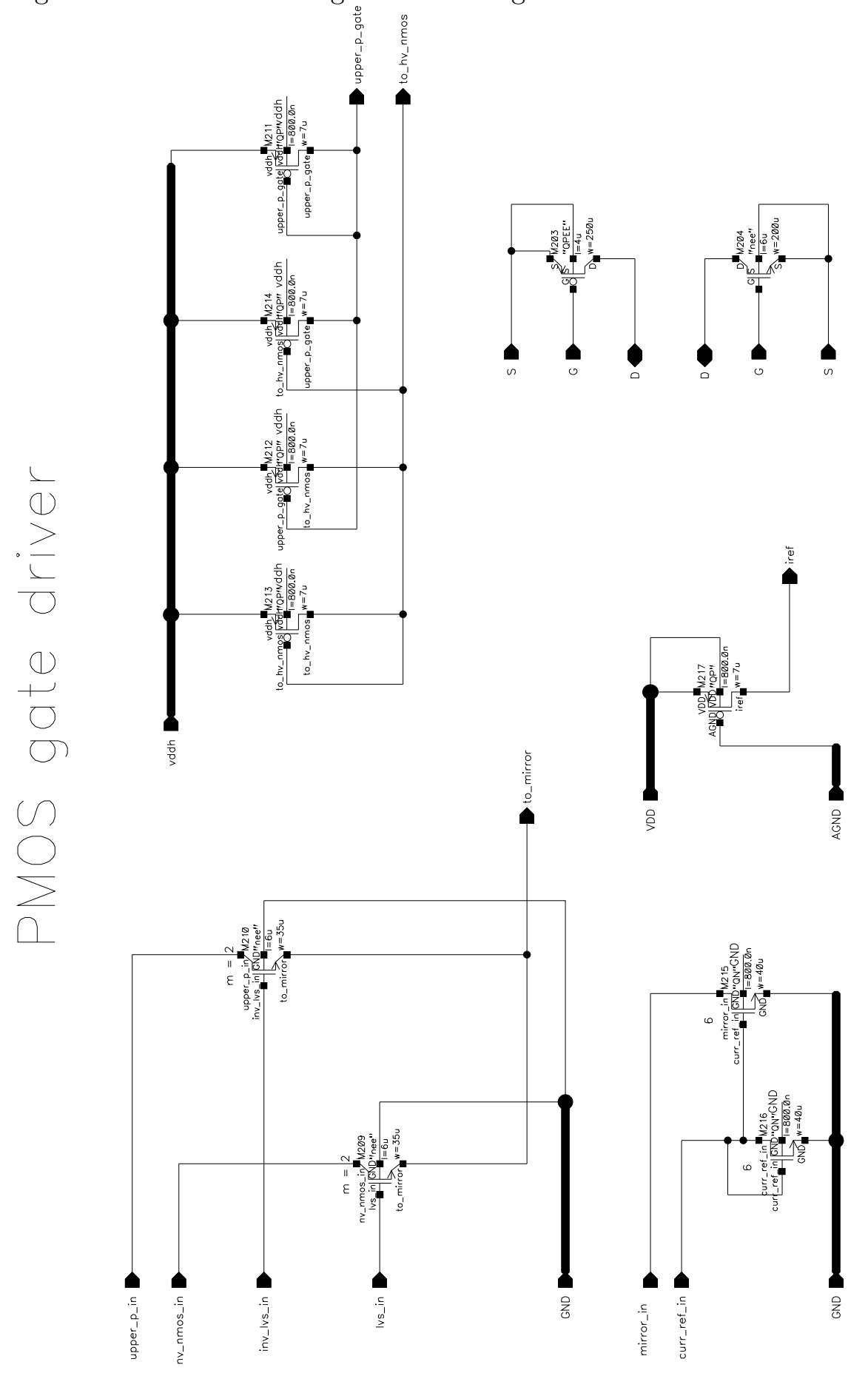


Figure D.6: Block diagram of M702 gate driver.

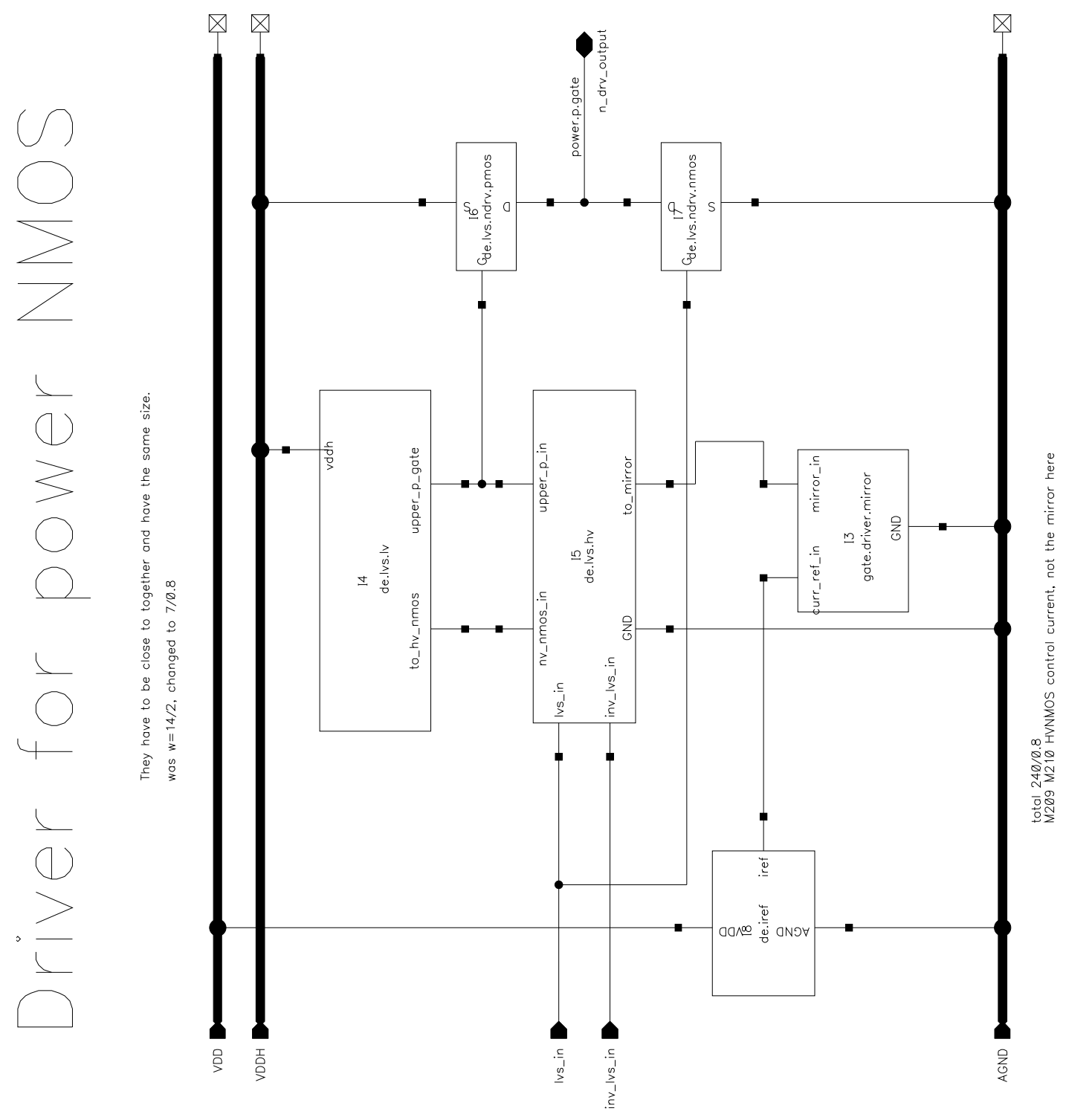


Figure D.7: Schematic diagram of M702 gate driver.

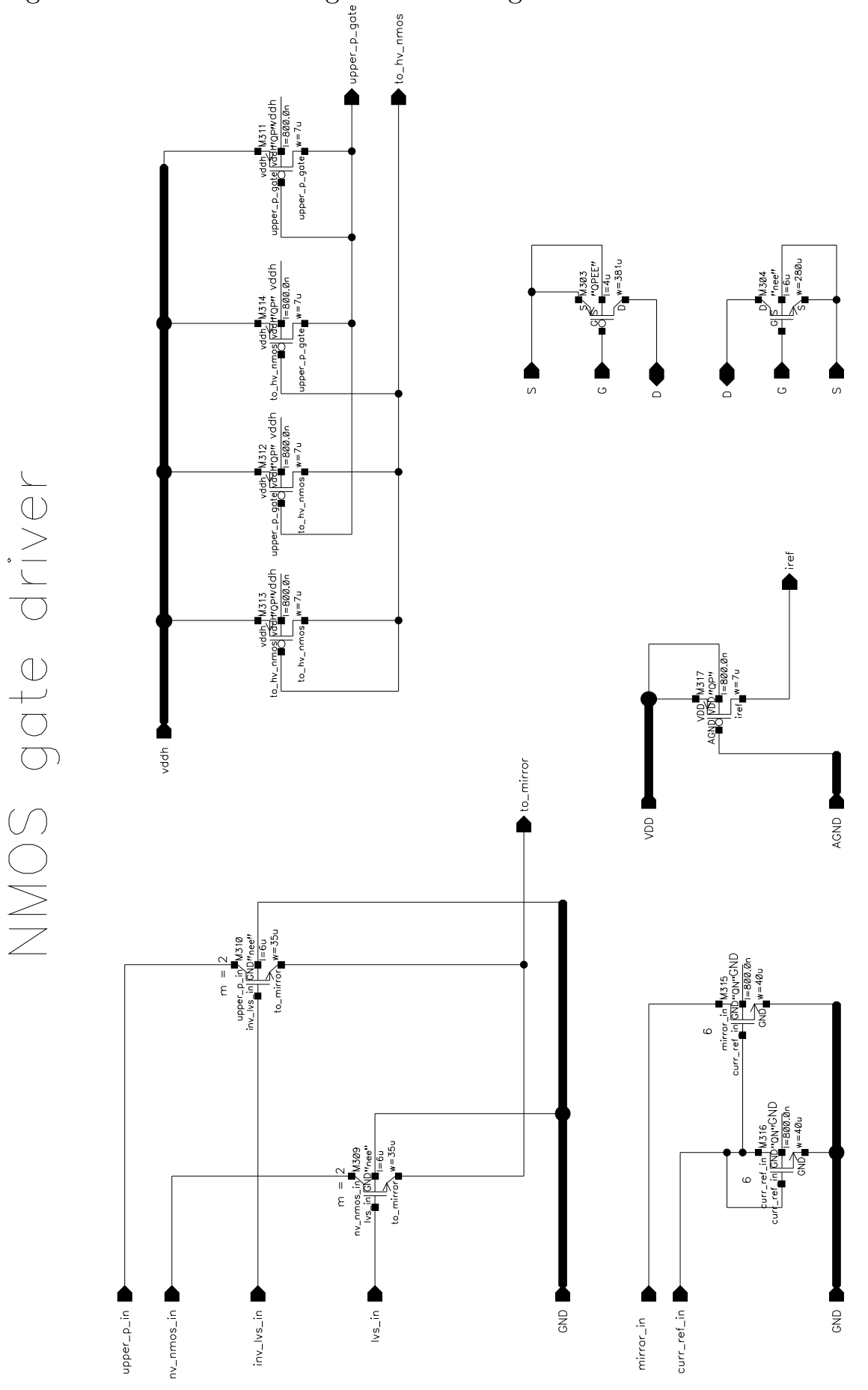


Figure D.8: Schematic diagram of the digital block.

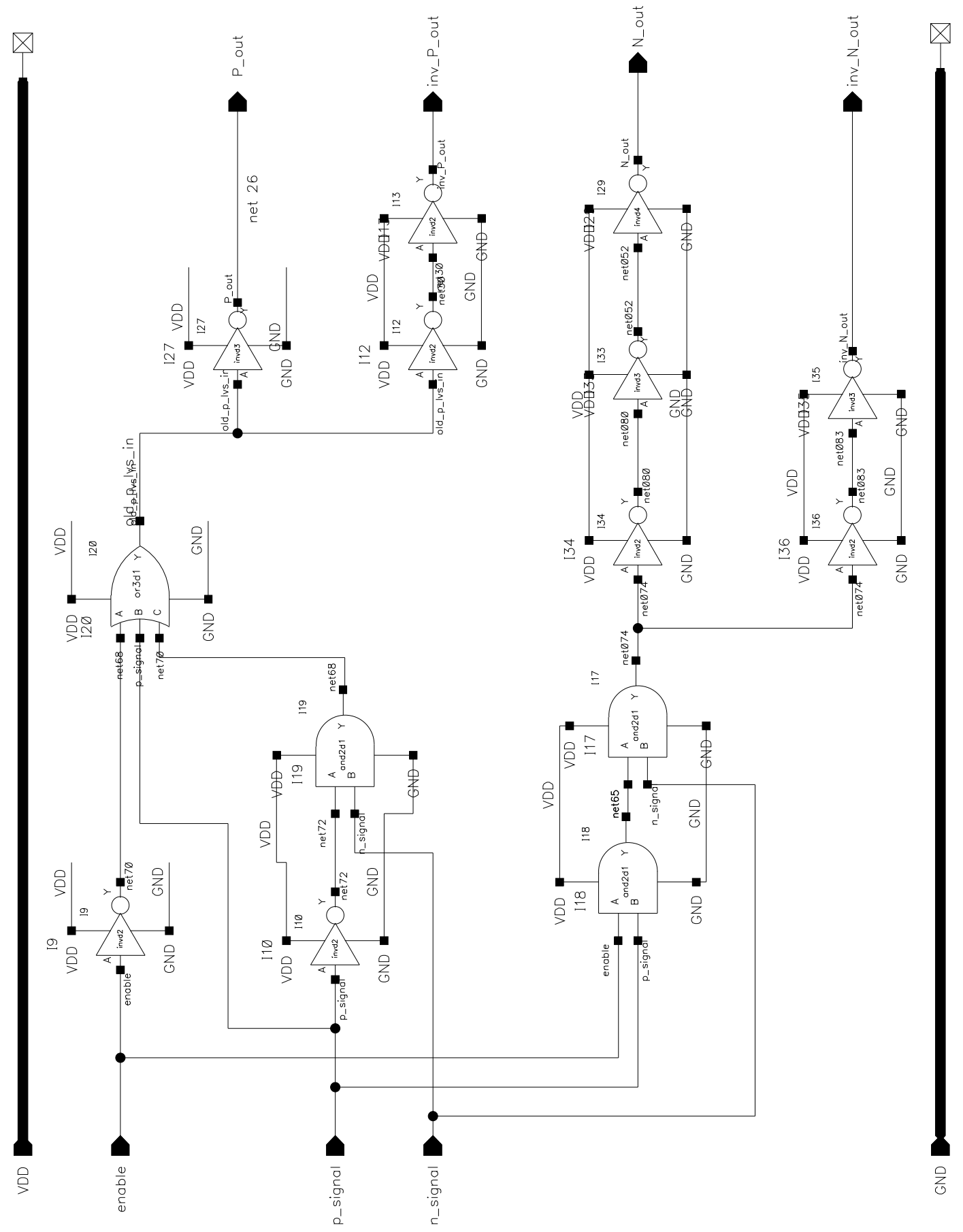


Figure D.9: Schematic diagram of the ultrasound transducer.

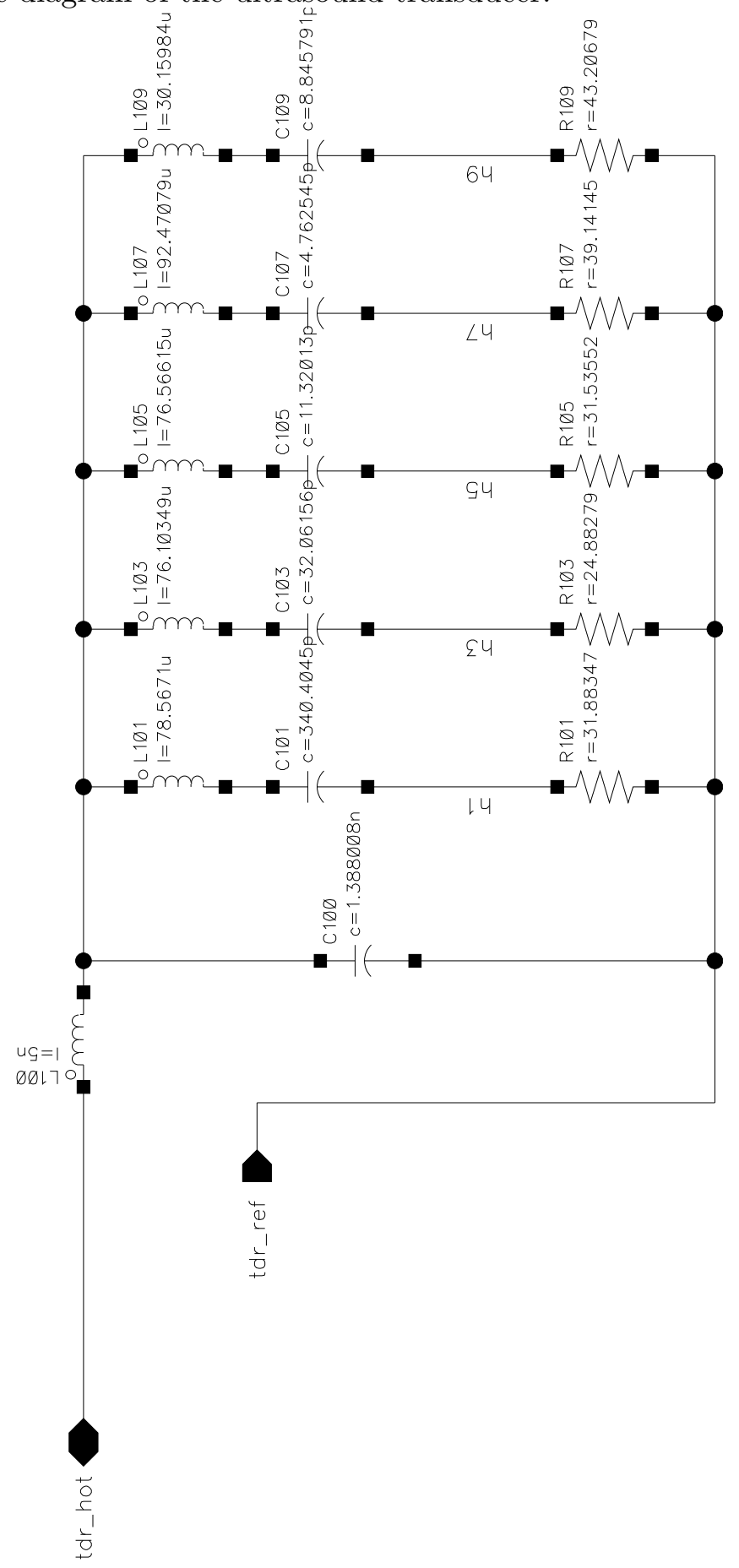


Appendix E

\section{Layout View}

The layout views used in Virtuoso for simulations. 
Figure E.1: Final integrated amplifier layout.

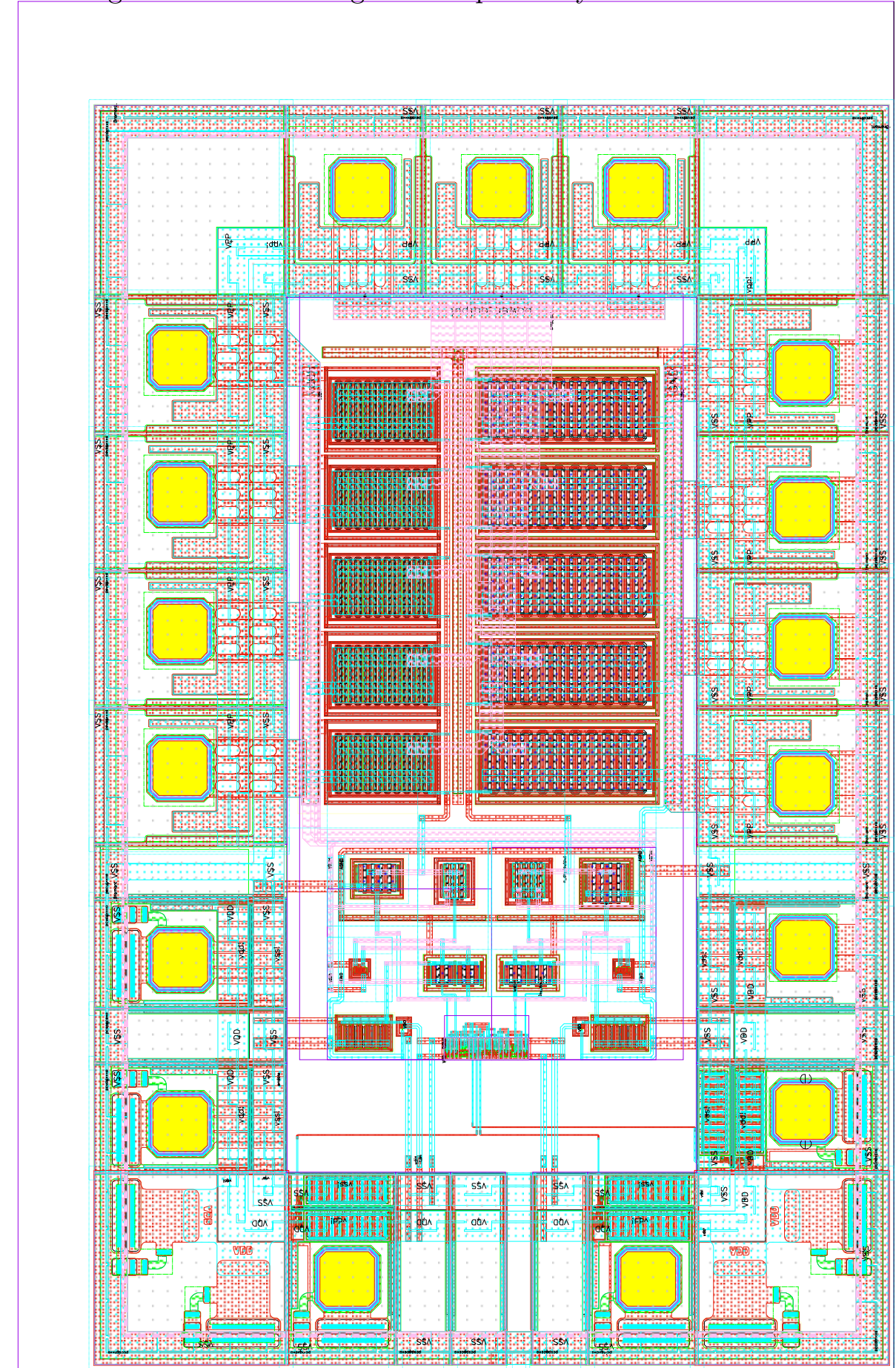


Figure E.2: Layout with M701 Gate driver.

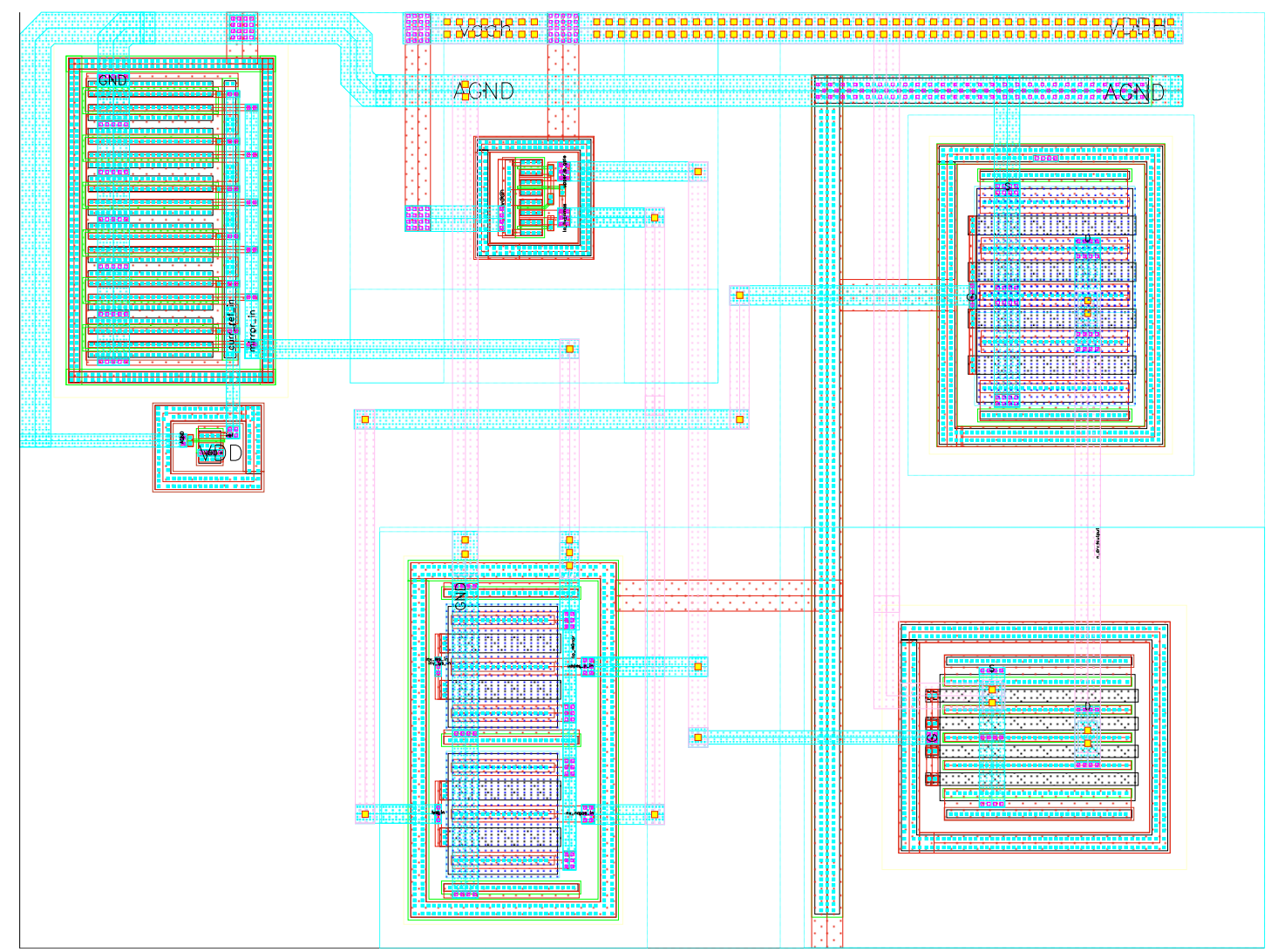


Figure E.3: Layout with M702 Gate driver.

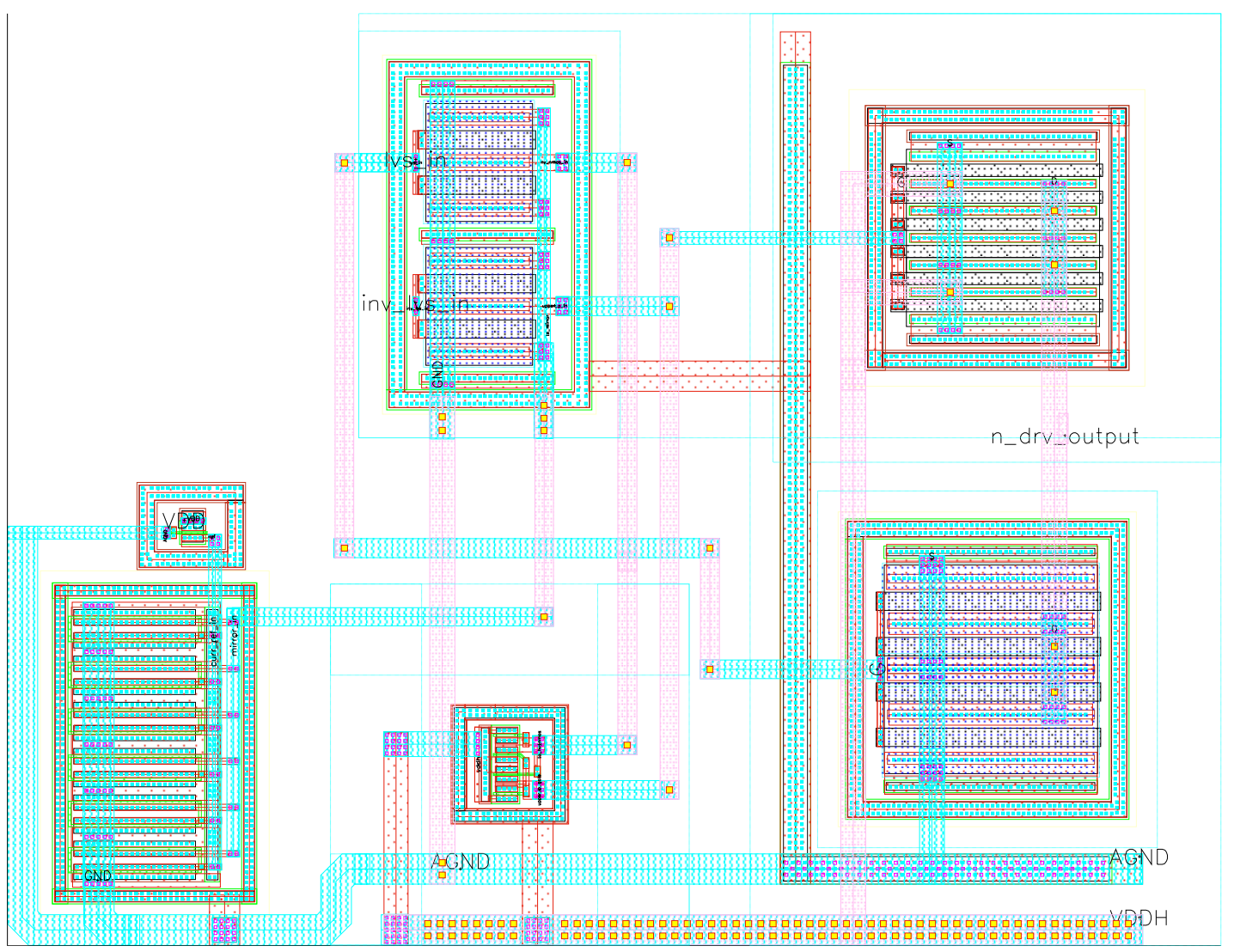


Figure E.4: Layout with M217 / M317.

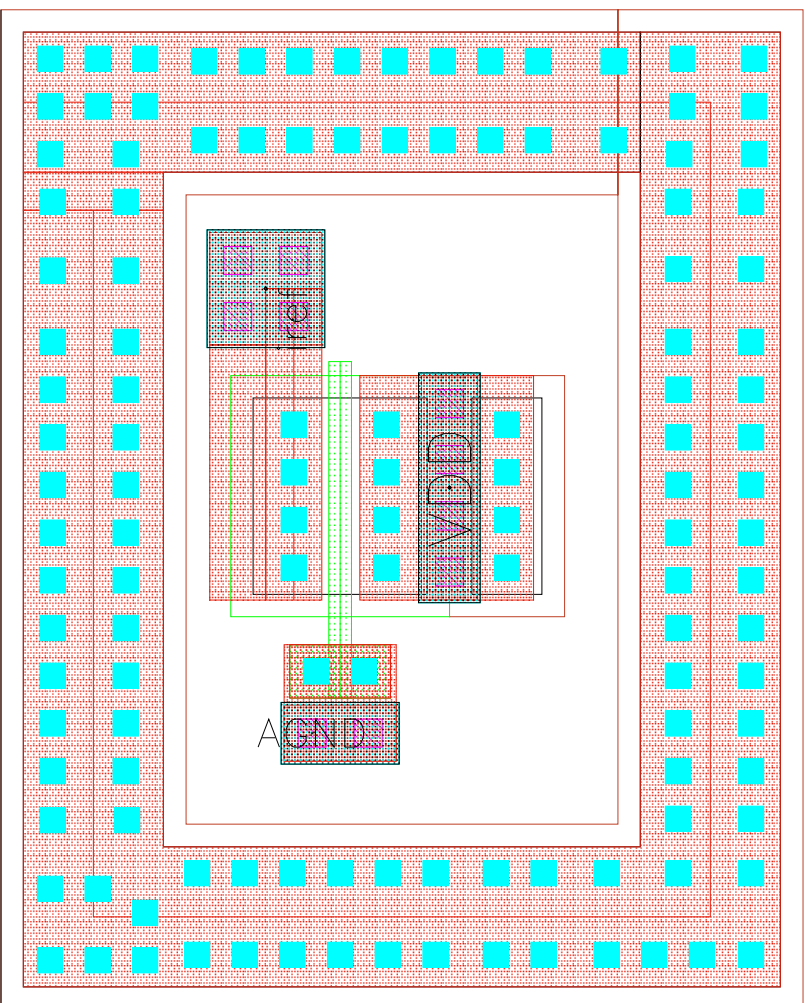


Figure E.5: Power transistors layout. Top: HV PMOS M701, bottom: HV NMOS M702
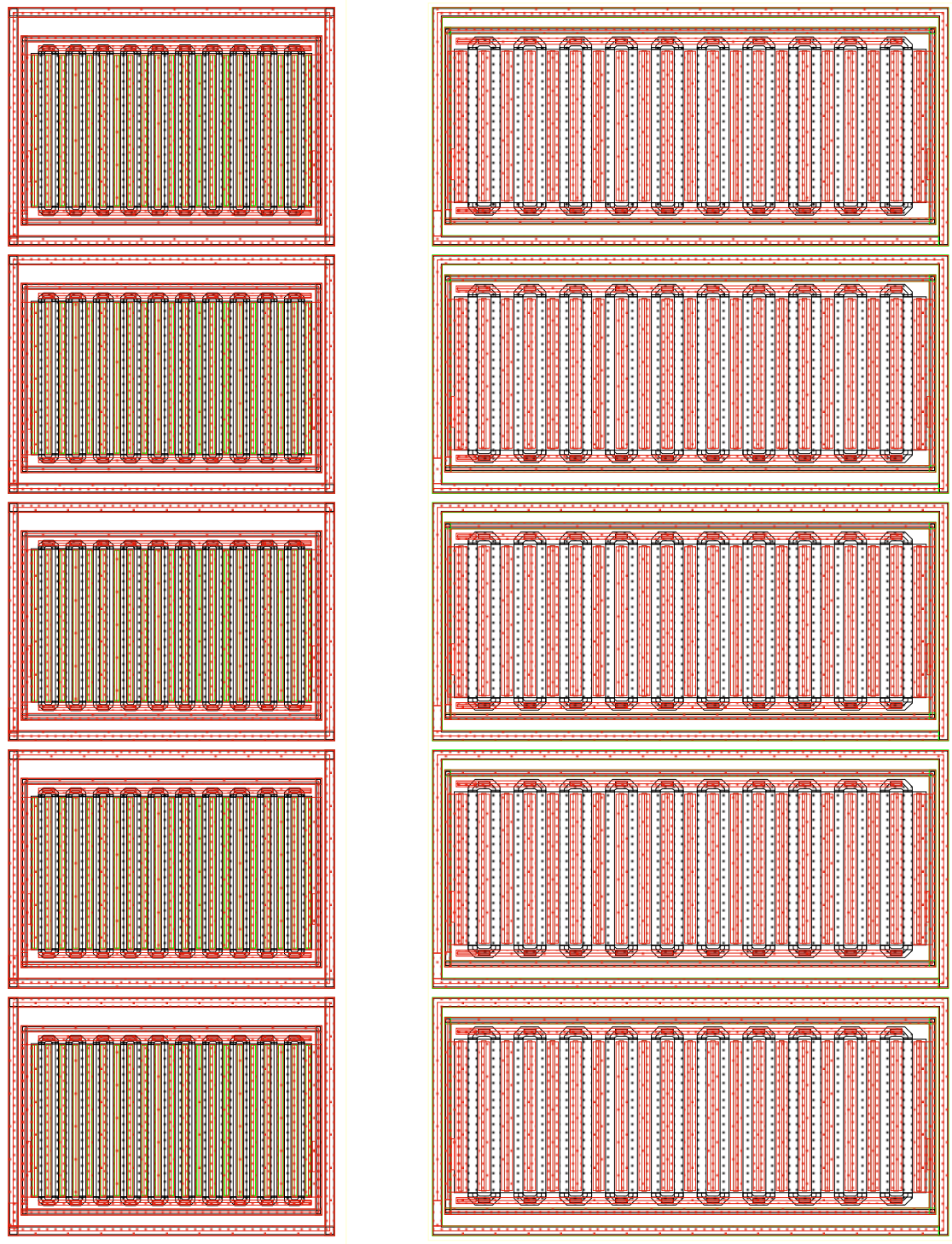
Figure E.6: Layout with transistor M203.

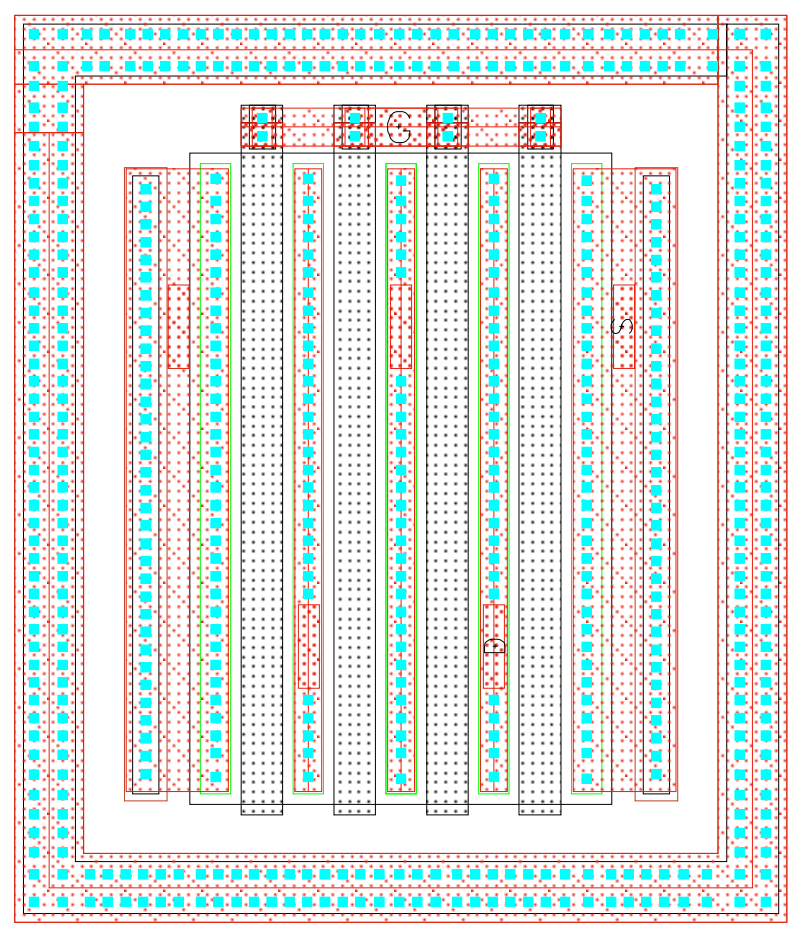


Figure E.7: Layout with transistor M204

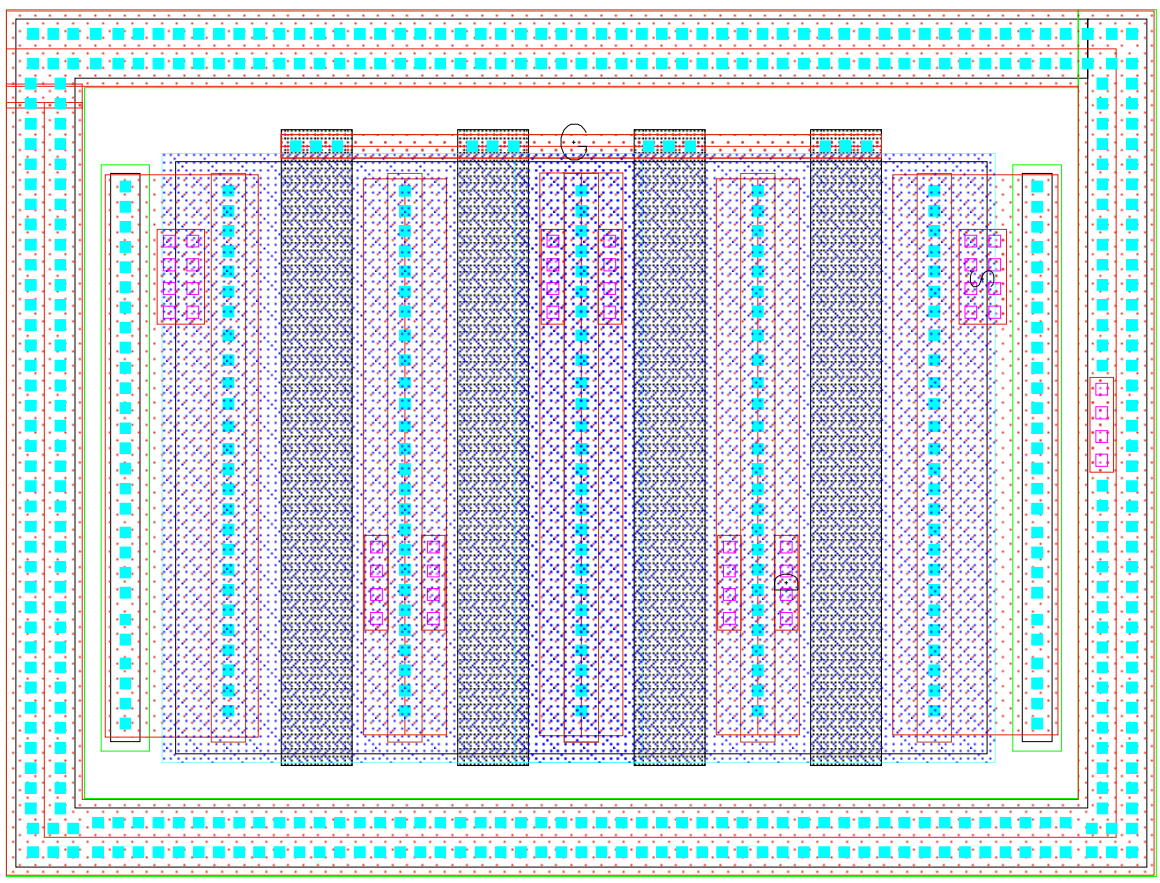


Figure E.8: Layout with transistor M303.

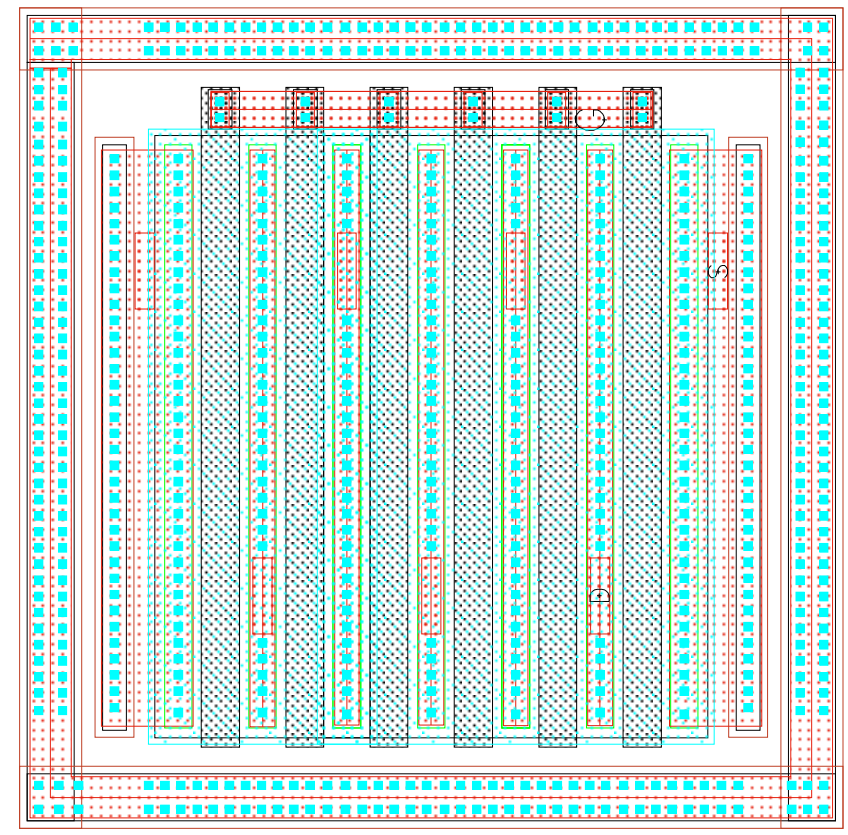


Figure E.9: Layout with transistor M304.

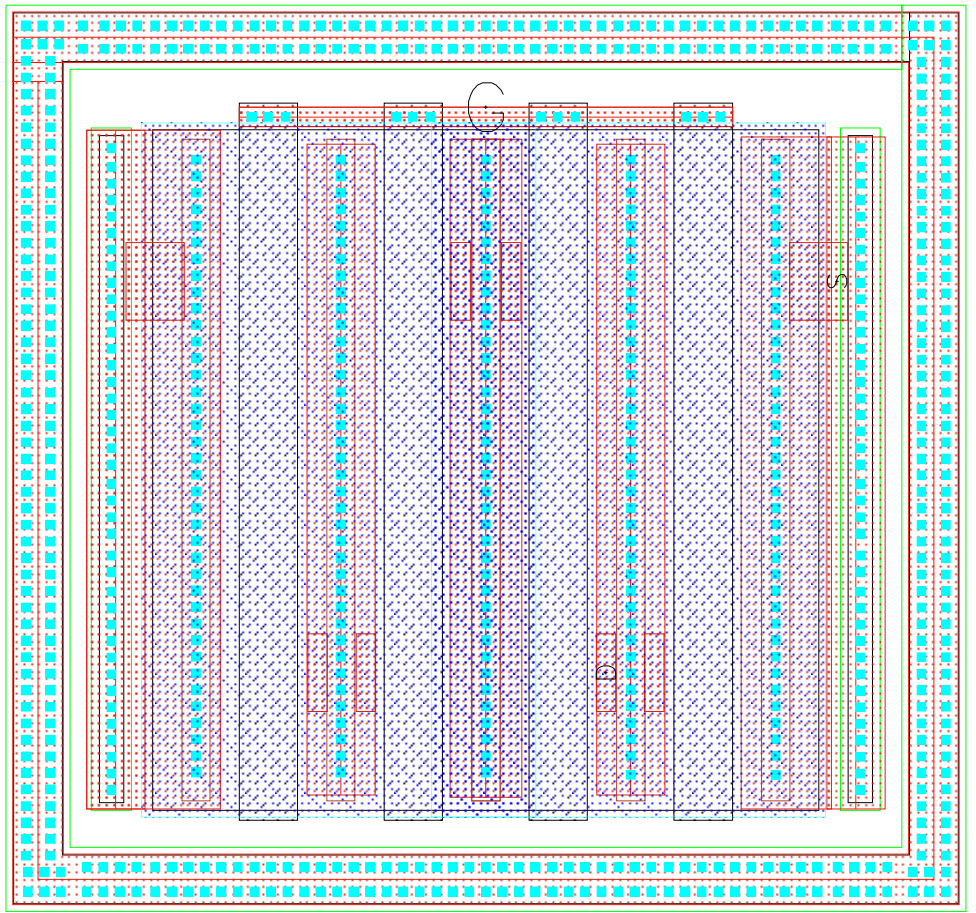


Figure E.10: Layout with transistors M209 / M309 and M210 / 310.

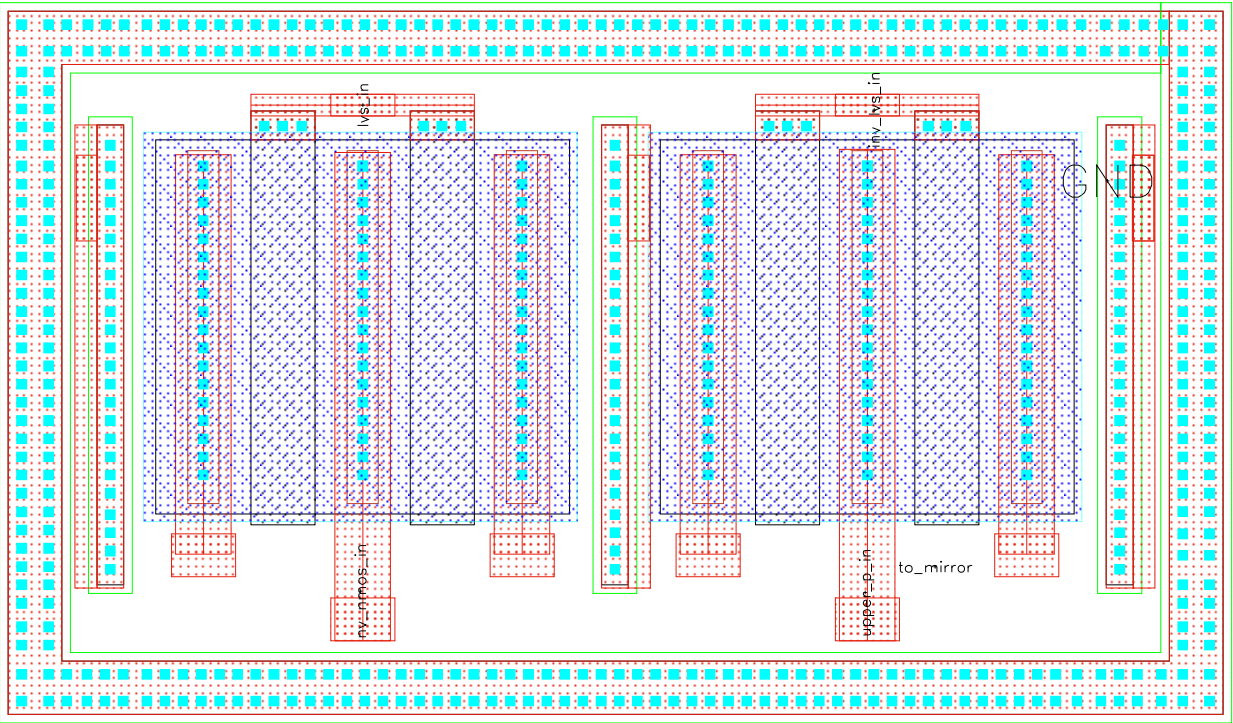


Figure E.11: Layout with M211 - M214 / M311 - M314.

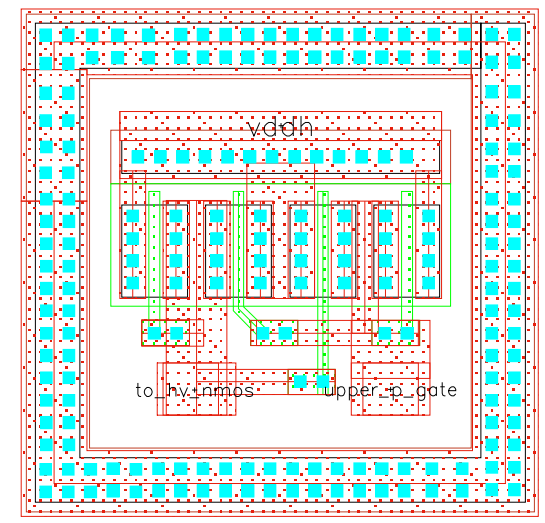


Figure E.12: Layout with current mirror, M215 / M315 and M216 / M316.

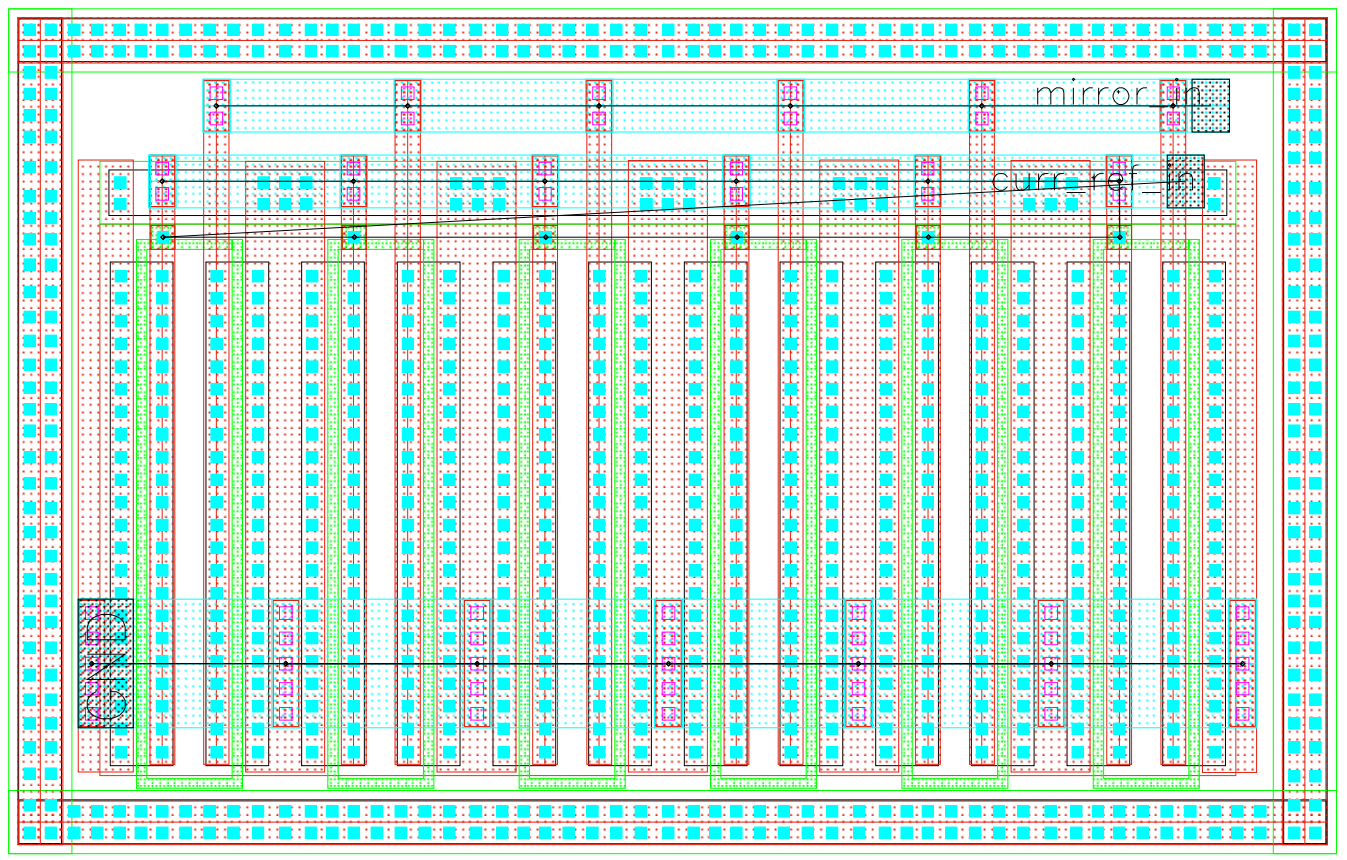


Figure E.13: Layout with combinational circuit block.

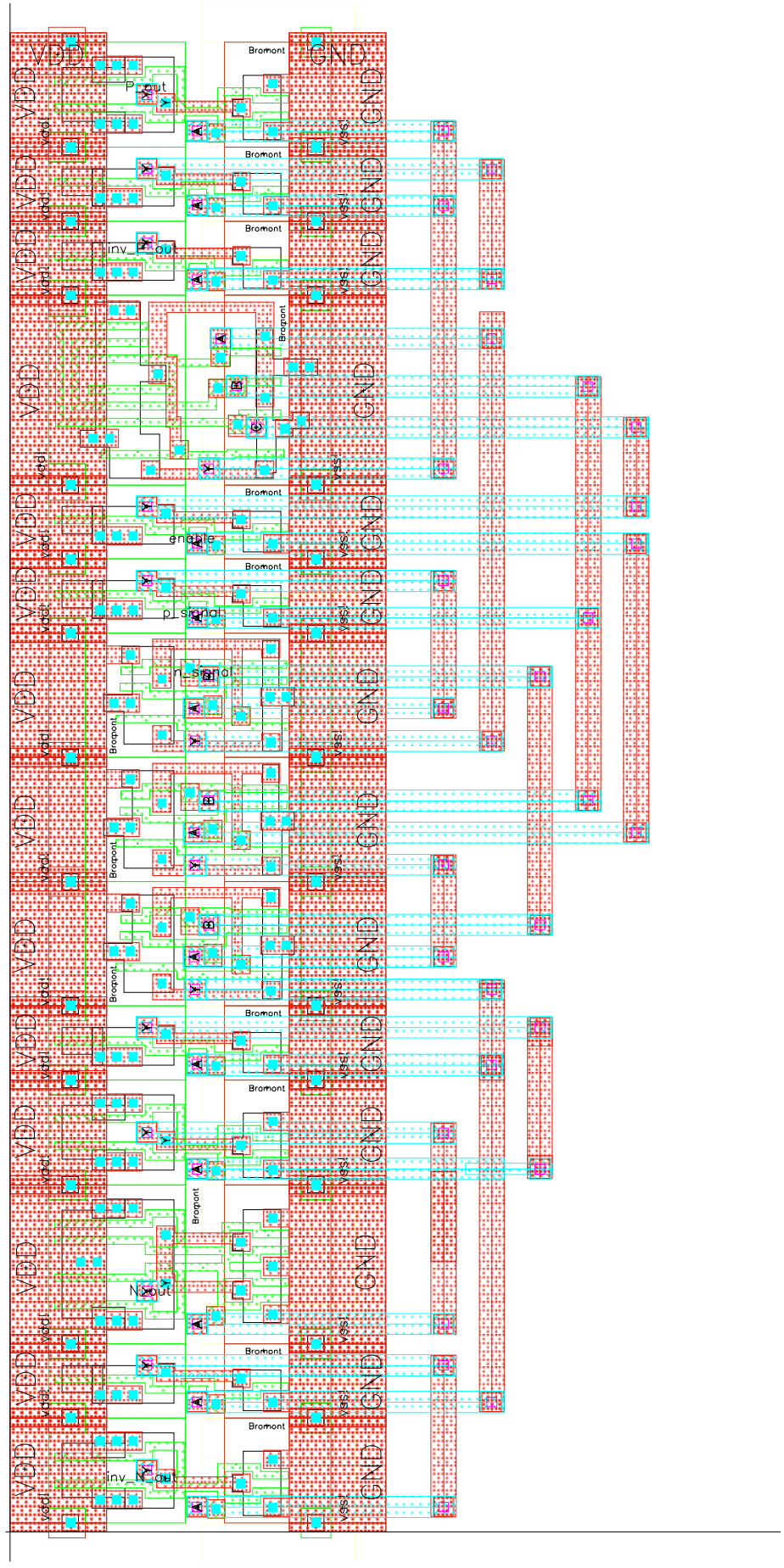




\section{References}

[1] G. Ter Haar, "Ultrasound focal beam surgery," Ultrasound in Medicine and Biology, vol. 21, no. 9, pp. 1089-1100, 1995.

[2] T. Hall and C. Cain, "A low cost compact 512 channel therapeutic ultrasound system for transcutaneous ultrasound surgery," AIP Conference Proceedings, vol. 829, no. 1, pp. 445-449, May 2006.

[3] N. Yu and R. Riener, "Review on MR-compatible robotic systems," in The First IEEE/RASEMBS International Conference on Biomedical Robotics and Biomechatronics, 2006. BioRob 2006, pp. 661-665.

[4] F. Ellinger "Power Amplifiers" in Radio frequency Integrated Circuits and Technologies, Berlin; New York: Springer, 2007, ch. 9, pp. 307-327.

[5] M. K. Kazimierczuk, RF Power Amplifiers, Chichester, West Sussex, U.K.: Wiley, 2008.

[6] L. Svilainis, V. Dumbrava and G. Motiejunas, "Optimization of the ultrasonic excitation stage, "30th International Conference on Information Technology Interfaces, 2008. ITI 2008. 2008. pp. $791-796$.

[7] Y. Mizutani, T. Suzuki, H. Ikeda and H. Yoshida, "Automatic frequency control for maximizing RF power fed to ultrasonic transducer operating at $1 \mathrm{MHz}$," Thirty-First IAS Annual Meeting in Industry Applications Conference, 1996, IAS '96, vol. 3, pp. 1585-1588

[8] K. Agbossou, J. Dion, S. Carignan, M. Abdelkrim and A. Cheriti, "Class D amplifier for a power piezoelectric load," IEEE Transactions on Ultrasonics, Ferroelectrics and Frequency Control, vol. 47, pp. 1036-1041, 2000. 
[9] L. Yang, L. Xu, T. Yang and B. Zhang, "Class D power amplifier for audio beam system," International Conference on Mechatronics and Automation, 200\%. ICMA 200\%. 200\%. pp. 34693474 .

[10] S. C. Tang and G. T. Clement, "A harmonic cancellation technique for an ultrasound transducer excited by a switched-mode power converter," in Proc. IEEE Ultrasonics Symp., 2008, pp. 20762079 .

[11] H. L. Cheng, C. A. Cheng, C. C. Fang and H. C. Yen, "Single-switch high power factor inverter for driving piezoelectric ceramic transducer", International Conference on Power Electronics and Drive Systems, 2009. PEDS 2009. pp. 1571-1576.

[12] G. Lewis and W. Olbricht, "Design and characterization of a high-power ultrasound driver with ultralow-output impedance," Review of Scientific Instruments, vol. 80, no. 11, p. 114704, 2009.

[13] W. T. Ang, C. Scurtescu, W. Hoy, T. El-Bialy, Y. Y. Tsui, and J. Chen, "Design and implementation of therapeutic ultrasound generating circuit for dental tissue formation and tooth-root healing," IEEE Trans. on Biomedical Circuits and Systems, vol. 4, no. 1, pp. 49-61, 2010.

[14] A. Arnau, Piezoelectric Transducers and Applications. 2nd ed. New York: Springer, 2008. ch.1, p. 18 , p. 23 , ch.18, sec. 18.3 , pp. 467-472.

[15] "IEEE Standard Definitions and Methods of Measurement for Piezoelectric Vibrators,” 1966.

[16] D. M. Pozar, "Transmission Line Theory," in Microwave Engineering, 3rd ed. New Jersey: John Wiley and Sons, 2005, p. 77.

[17] H. Koizumi; T. Suetsugu; M. Fujii; K. Shinoda; S. Mori; K. Iked, "Class DE high-efficiency tuned power amplifier," in IEEE Transactions on Circuits and Systems I: Fundamental Theory and Applications, vol.43, no.1, pp.51-60, Jan 1996.

[18] Foundry Design Kit $0.8 \mu m$ 5/20V CMOS User's Guide, Dalsa Semiconductor Inc. Document No., DK08EUG01.09, 2007.

[19] M. Albulet, "An exact analysis of Class-DE amplifier at any output Q," IEEE Trans. Circuits and Systems I, vol. 46, no. 10, pp. 1228-1239, Oct 1999. 
[20] H. Sekiya, T. Negishi, T. Suetsugu, and T. Yahagi, "Operation of Class DE amplifier outside optimum condition," in Proc. of IEEE International Symposium on Circuits and Systems (ISCAS 2006), May 2006, p. 4.

[21] H. Ballan and M. Declercq, "High-Voltage Analog and Digital Output Interfaces, "in, High Voltage Devices and Circuits in Standard CMOS Technologies, Dordrecht, Netherlands: Kluwer Academic Publishers, 1999, ch. 6, pp. 181-207.

[22] S. Kang and Y. Leblebici, "MOS Inverters: Switching Characteristics and Interconnect Effects," in CMOS Digital Integrated Circuits: Analysis and Design, 3rd ed. Boston, MA: McGraw-Hill, 2003, ch.6, sec 6.3, pp. 220-228.

[23] Foundry Design Kit $0.8 \mu m$ 5/20V CMOS Cell Library, Dalsa Semiconductor Inc., Document No. DK08EDS01.04, 2005. 34 D. Clein 1958-, CMOS IC Layout : Concepts, Methodologies, and Tools /. Boston : Newnes, c2000.

[24] "Audit Checklists", in CMOS IC Layout: concepts, methodologies, and tools. Boston, MA: Newnes, 2000, pp. 246-247. 